HD-THEP-95-7

February 1, 2008

\title{
Effective Action for the Chiral Quark-Meson Model*
}

\author{
D. - U. JUNGNICKEL ${ }^{\dagger}$ \\ and \\ C. Wetterich ${ }^{\ddagger}$ \\ Institut für Theoretische Physik \\ Universität Heidelberg \\ Philosophenweg 16 \\ 69120 Heidelberg, Germany
}

\begin{abstract}
The scale dependence of an effective average action for mesons and quarks is described by a nonperturbative flow equation. The running couplings lead to spontaneous chiral symmetry breaking. We argue that for strong Yukawa coupling between quarks and mesons the low momentum physics is essentially determined by infrared fixed points. This allows us to establish relations between various parameters related to the meson potential. The results for $f_{\pi}$ and $\langle\bar{\psi} \psi\rangle$ are not very sensitive to the poorly known details of the quark-meson effective action at scales where the mesonic bound states form. For realistic constituent quark masses we find $f_{\pi}$ around $100 \mathrm{MeV}$.
\end{abstract}

\footnotetext{
* Supported by the Deutsche Forschungsgemeinschaft

${ }^{\dagger}$ Email: D.Jungnickel@thphys.uni-heidelberg.de

${ }^{\ddagger}$ Email: C.Wetterich@thphys.uni-heidelberg.de
} 


\section{Introduction}

Quantum chromodynamics as the theory of strong interactions and its symmetries are well tested both for high and low momenta. For momenta $q^{2} \gtrsim(2 \mathrm{GeV})^{2}$ asymptotic freedom [1] permits the use of perturbation theory for a quark-gluon description with small gauge coupling $g_{s}$. The long distance behavior for $q^{2} \lesssim(300 \mathrm{MeV})^{2}$ can partially be described by chiral perturbation theory $[2,3]$. Here the picture is based on a nonlinear or linear $\sigma$-model [4] for the pseudo-scalar mesons. The latter can also be extended to describe in addition scalar, vector and pseudo-vector mesons. Such effective models incorporate the chiral symmetries of QCD and use several free couplings to parameterize the unknown strong interaction dynamics. The parameters are determined phenomenologically [3, 5], but on a more fundamental level the question arises how they can be related to the parameters of short distance QCD, i.e. the strong fine structure constant $\alpha_{s}=\frac{g_{s}^{2}}{4 \pi}$ and the current quark masses. For example, one may ask how the most prominent quantity of the mesonic picture, namely the pion decay constant $f_{\pi}$ which measures the strength of spontaneous chiral symmetry breaking, can be computed from $\alpha_{s}$ or vice versa.

Important progress in this question has been achieved by numerical simulations in lattice gauge theories [6]. Serious difficulties in this approach remain, however, related to the treatment of dynamical quarks and chiral symmetry. There is also a vast amount of literature on various analytical attempts to attack this problem. Examples can be found in [7]. In this paper we employ a new analytical method based on nonperturbative flow equations for scale dependent effective couplings. These couplings parameterize the effective average action $\Gamma_{k}$ [8] which is a type of coarse grained free energy. It includes the effects of all quantum fluctuations with momenta larger than an infrared cutoff $\sim k$. In the limit where the average scale $k$ tends to zero $\Gamma_{k \rightarrow 0}$ becomes therefore the usual effective action, i.e. the generating functional of $1 P I$ Green functions [9]. The scale dependence of $\Gamma_{k}$ can be described by an exact nonperturbative evolution equation $[10,9]$

$$
\frac{\partial}{\partial t} \Gamma_{k}[\varphi]=\frac{1}{2} \operatorname{Tr}\left\{\left(\Gamma_{k}^{(2)}[\varphi]+R_{k}\right)^{-1} \frac{\partial R_{k}}{\partial t}\right\}
$$

where $t=\ln (k / \Lambda)$ with $\Lambda$ some suitable high momentum scale. The trace represents here a momentum integration as well as a summation over internal indices and we note the appearance on the right hand side of the exact inverse propagator $\Gamma_{k}^{(2)}$ as given by the second functional variation of $\Gamma_{k}$ with respect to the field variables $\varphi$. The function $R_{k}(q)$ parameterizes the detailed form of the infrared cutoff or the averaging procedure. With the choice $^{1}$

$$
R_{k}(q)=\frac{Z_{\varphi, k} q^{2} e^{-q^{2} / k^{2}}}{1-e^{-q^{2} / k^{2}}}
$$

we observe that the momentum integration in eq. (1.1) is both infrared and ultraviolet finite. For fluctuations with small momenta $q^{2} \ll k^{2}$ the infrared cutoff $R_{k} \sim Z_{\varphi, k} k^{2}$ acts like an additional mass term in the propagator, whereas for $q^{2} \gg k^{2}$ it is ineffective.

\footnotetext{
${ }^{1} Z_{\varphi, k}$ is an appropriate wave function renormalization constant which will be specified later.
} 
The only difference between the flow equation (1.1) and the $k$-derivative of a one-loop expression with infrared cutoff $R_{k}$ concerns the appearance of $\Gamma_{k}^{(2)}$ instead of the second functional derivative of the classical action. This turns eq. (1.1) into an exact equation, but also transmutes it into a complicated functional differential equation which can only be solved approximately by truncating the most general form of $\Gamma_{k}$. As it should be, eq. (1.1) can be shown [11] to be equivalent to earlier forms of the exact renormalization group equation [12]. It may be interpreted as a differential form of the Schwinger-Dyson equations [13]. The difficult part is, however, not so much the establishment of an exact flow equation but rather the finding of a suitable nonperturbative truncation scheme which allows to solve the differential equation. Then the flow equation can be integrated from some short distance scale $\Lambda$, where $\Gamma_{\Lambda}$ can be taken as the classical action, to $k \rightarrow 0$ thus solving the model approximately.

Within the formalism of exact flow equations for the average action it is possible to change the relevant degrees of freedom [14]. The idea is now to start from the exact flow equations for quarks and gluons for $k>k_{\varphi}$, and to use a similar exact flow equation for quarks and mesons for $k<k_{\varphi}$. The transition at the scale $k_{\varphi}(600-700 \mathrm{MeV})$ between the two pictures can be encoded into an exact identity [14] which replaces multiquark interactions in the quark-gluon picture by mesonic interactions in the quark-meson picture. We emphasize that in the quark-meson description the quarks remain important degrees of freedom as long as $k$ is larger than a typical constituent quark mass $m_{q} \simeq$ $300 \mathrm{MeV}$. We have therefore to deal with an effective quark-meson model, where the mesons are described by a linear $\sigma$-model with Yukawa coupling $h$ to the quarks. A first attempt to describe the transition to a quark-meson model within a QCD-inspired model with four-quark interactions [14] has been very encouraging. Spontaneous chiral symmetry breaking was observed for low $k$, with a chiral condensate of the right order of magnitude. In the following, the formalism has been generalized to QCD [15], with a method where the gluonic fluctuations with $q^{2}>k^{2}$ are integrated out subsequently as $k$ is lowered. So far, the treatment of the quark-meson model for scales $k<k_{\varphi}$ has been very rough, however, since only quark fluctuations were included in ref. [14]. It is the purpose of this paper to present a systematic study of the scale dependence of the quark-meson effective action, including both quark and meson fluctuations.

Our main tool are nonperturbative flow equations which describe the change of shape of the effective meson potential and the running of the Yukawa coupling $h(k)$. In the perturbative limit these equations reproduce the running of the couplings $[16,17]$ in a $U_{L}(N) \times U_{R}(N)$ model, which has been investigated in the context of dynamical top quark condensation [18]. In our context $N$ stands for the number of quark flavors. The most important nonperturbative ingredient in the flow equations will turn out to be the appearance of effective mass threshold functions which account for the decoupling of modes with mass larger than $k$. The solution of our approximate flow equations allows us to express typical low momentum quantities like $f_{\pi}$ in terms of the "initial values" for the quark-meson model at the scale $k_{\varphi}$, as for example the meson mass term $\bar{m}^{2}\left(k_{\varphi}\right)$ or the wave function renormalization constant $Z_{\varphi}\left(k_{\varphi}\right)$.

Not too surprisingly, the effective quark-meson Yukawa coupling $h$ will turn out to be 
rather strong. This can easily be seen by noting that for $k \rightarrow 0$ this coupling is related to the ratio of a constituent quark mass $m_{q}$ to $f_{\pi}=93 \mathrm{MeV}$, namely

$$
h(k=0)=\frac{2 m_{q}}{f_{\pi}} \simeq 6.5
$$

For larger $k$ the Yukawa coupling must be even stronger, with a typical nonperturbative initial value $h^{2}\left(k_{\varphi}\right) / 16 \pi^{2} \gtrsim 1$. The presence of a strong coupling has important consequences for the predictive power of the quark-meson model. Generically, the system of flow equations exhibits (partial) infrared fixed points in the absence of a mass scale. Due to the large Yukawa interaction the couplings are driven very fast towards these fixed points and the system "looses its memory" on the detailed form of the initial values at $k_{\varphi}$. Despite the fact that the running is finally stopped by the formation of the chiral condensate this infrared stability implies that the low momentum quantities essentially depend only on one "relevant" parameter at the scale $k_{\varphi}$, i.e. the ratio $\bar{m}^{2}\left(k_{\varphi}\right) / k_{\varphi}^{2}$. Using the value (1.3) for $h(0)$ and $h\left(k_{\varphi}\right)$ between 12 and 100 we find in a simplified model

$$
f_{\pi} \simeq(83-100) \mathrm{MeV}
$$

in good agreement with the observed value $f_{\pi}=93 \mathrm{MeV}$. An estimate of the error as well as a more complete treatment including more accurately the effects of the chiral anomaly and the strange quark mass is postponed to future work.

Besides the exciting prospect of computing $f_{\pi}$ and other parameters of the low momentum meson interactions from QCD our approach seems also capable to deal with other issues. Once the parameters at the transition scale $k_{\varphi}$ are fixed either by a QCDcomputation or by fitting low momentum observational data, it is straightforward to study the quark-meson system at nonvanishing temperature. With methods described in ref. [19] the temperature dependence of $f_{\pi}$ or $\langle\bar{\psi} \psi\rangle$ can be investigated. For $T \lesssim k_{\varphi} / 2 \pi$ a study within the effective quark-meson model with $T$-independent initial values at $k_{\varphi}$ should be sufficient, whereas for larger temperatures the $T$-dependence of initial parameters like $\bar{m}^{2}\left(k_{\varphi}\right)$ starts to become an important effect. One may therefore hope to gain new insight into the nature of the chiral phase transition in QCD [20]. Another interesting issue concerns the use of quark-meson models to describe hadronization in high energy scattering experiments involving quarks or gluons [21]. Here our approach may help to compute the phenomenological parameters used in those models. Finally, the scalar-fermion models have been extensively studied in the large $-N_{c}$ limit $[17,18]$, and our nonperturbative flow equations may help to access smaller values of $N_{c}$.

Our paper is structured as follows: in section 2 we give a brief phenomenological introduction to the chiral quark-meson model with $N$ flavors. The scale dependence of the effective meson potential is then described in section 3 and the scalar wave function renormalization can be found in section 4 . In section 5 we derive the $\beta$-function for the running Yukawa coupling between quarks and mesons as well as the quark wave function renormalization. Section 6 is devoted to a short discussion of the chiral anomaly and the presentation of two simplified models with two quark flavors. The first model is based 
on the symmetry $U_{L}(2) \times U_{R}(2)$ and neglects the effects of the chiral anomaly, whereas the second one based on $O(4)$ neglects all scalars whose masses obtain contributions from the chiral anomaly. In section 7 we discuss in detail the infrared stability properties for models with strong Yukawa couplings and the consequences for the "prediction" of $f_{\pi}$. Section 8 finally contains our quantitative estimates for $f_{\pi}$ and the chiral condensate $\langle\bar{\psi} \psi\rangle_{0}$. Conclusions are drawn in section 9 .

\section{The chiral quark-meson model}

We describe the low-energy degrees of freedom of QCD by an effective action for quarks and mesons. We concentrate in this paper on pseudo-scalar and scalar mesons $\varphi$ which transform in the $(\overline{\mathbf{N}}, \mathbf{N})$ representation of the flavor symmetry group $S U_{L}(N) \times S U_{R}(N)$ for $N$ flavors. We consider the chiral limit where the current quark masses are neglected. In its simplest form the effective action $\Gamma_{k}$ for quarks and mesons contains kinetic terms, a potential for the scalar fields and a Yukawa coupling between quarks and mesons:

$$
\begin{aligned}
\Gamma_{k} & =\int d^{4} x\left\{Z_{\varphi}(k) \partial_{\mu} \varphi_{a b}^{*} \partial^{\mu} \varphi^{a b}+U_{k}\left(\varphi, \varphi^{\dagger}\right)\right. \\
& \left.+i Z_{\psi}(k) \bar{\psi}^{a} \gamma^{\mu} \partial_{\mu} \psi_{a}+\bar{h}(k) \bar{\psi}^{a}\left[\frac{1+\bar{\gamma}}{2} \varphi_{a}{ }^{b}-\frac{1-\bar{\gamma}}{2}\left(\varphi^{\dagger}\right)_{a}{ }^{b}\right] \psi_{b}\right\} .
\end{aligned}
$$

Our Euclidean conventions $(\bar{h}(k)$ is real) are specified in appendix A. The scalar potential is assumed to be a function of the invariants

$$
\begin{aligned}
\rho & =\operatorname{tr}\left(\varphi^{\dagger} \varphi\right) \\
\tau_{2} & =\frac{N}{N-1} \operatorname{tr}\left(\varphi^{\dagger} \varphi\right)^{2}-\frac{1}{N-1} \rho^{2} \\
\xi & =\operatorname{det} \varphi+\operatorname{det} \varphi^{\dagger}
\end{aligned}
$$

where we neglect the dependence on additional higher order invariants present for $N \geq 3$ (cf. appendix B).

Spontaneous chiral symmetry breaking with a residual vector-like $S U(N)$ flavor symmetry occurs if the potential has a minimum for $\sigma_{0} \neq 0$

$$
\varphi_{0}=\left(\begin{array}{cccc}
\sigma_{0} & & & \\
& \sigma_{0} & & \\
& & \ddots & \\
& & & \sigma_{0}
\end{array}\right), \quad \rho_{0}=N\left|\sigma_{0}\right|^{2} .
$$

In this case we consider a quartic approximation for the potential

$$
U_{k}=-\bar{\mu}^{2}(k) \rho+\frac{1}{2} \bar{\lambda}_{1}(k) \rho^{2}+\frac{N-1}{4} \bar{\lambda}_{2}(k) \tau_{2}-\frac{1}{2} \bar{\nu}(k) \xi
$$


where $\bar{\mu}^{2}(k)$ is related to the $k$-dependent minimum value $\rho_{0}(k)$ by

$$
\bar{\mu}^{2}(k)=\bar{\lambda}_{1}(k) \rho_{0}(k)-\frac{|\bar{\nu}(k)|}{2}\left(\frac{\rho_{0}(k)}{N}\right)^{\frac{N-2}{2}} .
$$

Without loss of generality we will restrict ourselves to positive $\bar{\nu}$. Up to an irrelevant constant we can also write

$$
U_{k}=\frac{1}{2} \bar{\lambda}_{1}(k)\left(\rho-\rho_{0}(k)\right)^{2}+\frac{N-1}{4} \bar{\lambda}_{2}(k) \tau_{2}-\frac{1}{2} \bar{\nu}(k) \xi+\frac{1}{2} \bar{\nu}(k)\left(\frac{\rho_{0}(k)}{N}\right)^{\frac{N-2}{2}} \rho .
$$

On the other hand, at short distance scales spontaneous chiral symmetry breaking is not yet visible and $U_{k}$ is in the symmetric regime $\left(\sigma_{0}=0\right)$, where we use the parameterization

$$
U_{k}=\bar{m}^{2}(k) \rho+\frac{1}{2} \bar{\lambda}_{1}(k) \rho^{2}+\frac{N-1}{4} \bar{\lambda}_{2}(k) \tau_{2}-\frac{1}{2} \bar{\nu}(k) \xi .
$$

With these approximations our model can be described in terms of the renormalized couplings

$$
\begin{aligned}
h(k) & =Z_{\varphi}^{-1 / 2}(k) Z_{\psi}^{-1}(k) \bar{h}(k) \\
\lambda_{1,2}(k) & =Z_{\varphi}^{-2}(k) \bar{\lambda}_{1,2}(k)
\end{aligned}
$$

and either the mass term

$$
m^{2}(k)=Z_{\varphi}^{-1}(k) \bar{m}^{2}(k)
$$

or the location of the potential minimum

$$
\rho_{R}(k)=Z_{\varphi}(k) \rho_{0}(k) .
$$

The dimension of $\bar{\nu}$ depends on $N$ and the renormalized coupling is

$$
\nu_{R}(k)=Z_{\varphi}^{-\frac{N}{2}}(k) \bar{\nu}(k) .
$$

For $\bar{\nu}=0$ the model has an additional axial $U_{A}(1)$ symmetry which, however, is broken in QCD through the axial anomaly.

The quark-meson model is supposed to be obtained as an effective model at some scale $k_{\varphi}$, say $k_{\varphi} \simeq 600 \mathrm{MeV}$. It should be derivable from QCD by integrating out the gluonic degrees of freedom and converting nonlocal four-quark interactions into an effective quark-meson theory by the change of variables described in [14]. This gives a direct relation between $\varphi$ and a suitably defined [14] composite quark bilinear operator $\mathcal{O}_{a}^{b}=\left\langle\bar{\psi}^{b} \psi_{a}\right\rangle$ according to

$$
\mathcal{O}_{a}^{b}=\frac{2 \bar{m}^{2}\left(k_{\varphi}\right) Z_{\psi}\left(k_{\varphi}\right)}{\bar{h}\left(k_{\varphi}\right)} \varphi_{a}^{b}
$$

The aim of this paper is to follow the evolution of $\Gamma_{k}$ from the "initial value" at $k=k_{\varphi}$ to $k=0$. The effective action $\Gamma=\Gamma_{k=0}$ then describes the 1PI Green functions for the 
collective meson fields or quark bilinears. In particular, the chiral condensate is related to the vacuum expectation value of $\varphi$ corresponding to the minimum of the effective potential $U=U_{k=0}$ through $^{2}$

$$
\langle\bar{\psi} \psi\rangle_{0}=\frac{2 \bar{m}^{2}\left(k_{\varphi}\right) Z_{\psi}\left(k_{\varphi}\right)}{\bar{h}\left(k_{\varphi}\right)} \sigma_{0}
$$

with $^{3}$

$$
\sigma_{0}=\left(\frac{\rho_{0}(k=0)}{N}\right)^{1 / 2} .
$$

For $\sigma_{0}$ different from zero the chiral symmetry is spontaneously broken and the spectrum of scalars contains $N^{2}-1$ Goldstone bosons corresponding to the pions. Their interactions are described by the nonlinear $\sigma$-model. Neglecting the explicit $S U_{V}(N)$ breaking through quark masses the pion decay constant $f_{\pi}$ is given in our conventions by

$$
f_{\pi}=2 \sigma_{R}
$$

with renormalized expectation value

$$
\sigma_{R}=Z_{\varphi}^{1 / 2}(k=0) \sigma_{0}=\left(\frac{Z_{\varphi}(0) \rho_{0}(0)}{N}\right)^{1 / 2} .
$$

In our normalization the experimental value reads $f_{\pi}=93 \mathrm{MeV}$ or

$$
\sigma_{R}=46.5 \mathrm{MeV} \text {. }
$$

Another interesting quantity in our picture is the renormalized quark mass $(h \equiv h(0))$

$$
m_{q}=h \sigma_{R}
$$

This corresponds to a constituent mass generated by chiral symmetry breaking. (We remind that we consider the approximation of vanishing current quark masses here.) A typical value should be around $300 \mathrm{MeV}$ and the renormalized Yukawa coupling therefore be relatively large, $h \approx 6.5$.

The scalar spectrum is discussed in detail in appendix B. For $\sigma_{R} \neq 0$ the meson sector contains besides the $N^{2}-1$ massless Goldstone bosons the $\sigma$-field (radial mode). With $\lambda_{1}=\lambda_{1}(0)$ and $\nu_{R}=\nu_{R}(0)$ its mass is given by

$$
m_{\sigma}^{2}=2 N \lambda_{1} \sigma_{R}^{2}-\frac{1}{2} \nu_{R}(N-2) \sigma_{R}^{N-2} .
$$

One meson acquires a mass through the chiral anomaly. For the realistic case of $N=3$ this can be identified with the $\eta^{\prime}$ meson whereas the $\eta$ meson remains massless in the

\footnotetext{
${ }^{2}$ We note that $(2.13)$ defines the quark condensate at the scale $k_{\varphi}$ which may be different from the scale of usual chiral perturbation theory estimates.

${ }^{3}$ Neglecting fermion masses the phase of $\sigma_{0}$ is arbitrary and we employ here a real and positive $\sigma_{0}$. Correspondingly, $\langle\bar{\psi} \psi\rangle_{0}$ stands only for the magnitude of the chiral condensate.
} 
chiral limit as one of the Goldstone bosons. For $N=2$ we are left with the three pions as massless degrees of freedom whereas the $K$-mesons and the $\eta$-meson are absent from the spectrum. We will also for $N=2$ associate the anomalously massive meson with the $\eta^{\prime}$ meson. In our model its mass is given by

$$
m_{\eta^{\prime}}^{2}=\frac{N}{2} \nu_{R} \sigma_{R}^{N-2} .
$$

The remaining $N^{2}-1$ massive scalar fields in the adjoint representation of the diagonal flavor symmetry group $S U(N)$ have mass (for $\lambda_{2}=\lambda_{2}(0)$ )

$$
m_{a}^{2}=N \lambda_{2} \sigma_{R}^{2}+\nu_{R} \sigma_{R}^{N-2} .
$$

The neutral component can be associated with the $a_{0}$ meson with mass $983 \mathrm{MeV}$. For realistic meson masses $\left(m_{\eta^{\prime}}=958 \mathrm{MeV}\right)$ the couplings would be

$$
\begin{gathered}
\nu_{R} \simeq(958 \mathrm{MeV})^{2} \text { for } N=2 \\
\nu_{R} \simeq 13158 \mathrm{MeV} \text { for } N=3 \\
\lambda_{2} \simeq 11 \text { for } N=2 \\
\lambda_{2} \simeq 55 \text { for } N=3 .
\end{gathered}
$$

One should, however, notice that the values for $\nu_{R}$ and $\lambda_{2}$ are rather sensitive to the precise association of $m_{a}$ or $m_{\eta^{\prime}}$ with known particle masses and should therefore only be taken as a rough estimate. In particular, for $N=3$ the effects of a nonzero strange quark mass have to be incorporated for a more realistic estimate.

\section{Scale dependence of the effective meson potential}

The meson degrees of freedom can be introduced [14] at some short distance scale $k_{\varphi}$ by inserting the identity

$$
\begin{aligned}
1 & =\operatorname{const} \int \mathcal{D} \sigma_{A} \mathcal{D} \sigma_{H} \exp \left\{-\frac{1}{2}\left[\left(\sigma_{A}^{\dagger}-K_{A}^{\dagger} \tilde{G}-\mathcal{O}^{\dagger}[\psi] \tilde{G}\right) \tilde{G}^{-1}\left(\sigma_{A}-\tilde{G} K_{A}-\tilde{G} \mathcal{O}[\psi]\right)\right.\right. \\
& \left.\left.+\left(\sigma_{H}^{\dagger}-K_{H}^{\dagger} \tilde{G}-\mathcal{O}^{(5) \dagger}[\psi] \tilde{G}\right) \tilde{G}^{-1}\left(\sigma_{H}-\tilde{G} K_{H}-\tilde{G} \mathcal{O}^{(5)}[\psi]\right)\right]\right\}
\end{aligned}
$$

into the functional integral for the effective average action for quarks. Here $K_{A, H}$ are sources for the collective fields and correspond to the antihermitian and hermitian parts ${ }^{4}$ of $\varphi$. They are associated to the fermion bilinear operators $\mathcal{O}[\psi], \mathcal{O}^{(5)}[\psi]$ whose Fourier components read

$$
\begin{array}{r}
\mathcal{O}_{b}^{a}(q)=-i \int \frac{d^{4} p}{(2 \pi)^{4}} g(-p, p+q) \bar{\psi}^{a}(p) \psi_{b}(p+q) \\
\mathcal{O}^{(5) a}{ }_{b}(q)=-\int \frac{d^{4} p}{(2 \pi)^{4}} g(-p, p+q) \bar{\psi}^{a}(p) \bar{\gamma} \psi_{b}(p+q) .
\end{array}
$$

\footnotetext{
${ }^{4}$ The fields $\bar{\sigma}_{A, H}$ associated to $K_{A, H}$ by a Legendre transformation obey $\bar{\sigma}_{A}=-\frac{i}{2}\left(\varphi-\varphi^{\dagger}\right), \bar{\sigma}_{H}=$ $\frac{1}{2}\left(\varphi+\varphi^{\dagger}\right)$.
} 
The wave function renormalization $g(-p, p+q)$ and the propagator $\tilde{G}(q)$ are chosen such that the four-quark interaction contained in (3.1) cancels the dominant part of the QCDinduced nonlocal four-quark interaction in the effective average action formulated only for quarks. As a result, the introduction of collective fields by (3.1) replaces the dominant part of the four-quark interaction by terms quadratic and linear in the meson field. The resulting effective quark-meson interactions are more general than those of the model described in the last section. The momentum dependence of the kinetic terms and the Yukawa couplings can be described by an extended truncation of the effective average action which, for general space-time dimensions $d$, is given by

$$
\begin{aligned}
\Gamma_{k} & =\int d^{d} x U_{k}\left(\varphi, \varphi^{\dagger}\right) \\
& +\int \frac{d^{d} q}{(2 \pi)^{d}}\left\{Z_{\varphi, k}(q) q^{2} \operatorname{tr}\left(\varphi^{\dagger}(q) \varphi(q)\right)+Z_{\psi, k}(q) \bar{\psi}(q) \gamma^{\mu} q_{\mu} \psi(q)\right. \\
& \left.+\int \frac{d^{d} q}{(2 \pi)^{d}} \bar{h}_{k}(-q, q-p) \bar{\psi}(q)\left(\frac{1+\bar{\gamma}}{2} \varphi(p)-\frac{1-\bar{\gamma}}{2} \varphi^{\dagger}(-p)\right) \psi(q-p)\right\} .
\end{aligned}
$$

At the scale $k_{\varphi}$ the average potential is then purely quadratic

$$
U_{k_{\varphi}}=\bar{m}^{2} \operatorname{tr}\left(\varphi^{\dagger} \varphi\right)
$$

and the inverse scalar propagator is related to $\tilde{G}(q)$ in eq. (3.1) by

$$
\begin{aligned}
\tilde{G}^{-1}(q) & =2 \bar{m}^{2}+2 \bar{Z}_{\varphi}(q) q^{2} \\
\bar{Z}_{\varphi}(q) & \equiv Z_{\varphi, k_{\varphi}}(q)
\end{aligned}
$$

The initial value of the Yukawa coupling corresponds to the "quark wave function in the meson" in eq. (3.1), i.e.

$$
\bar{h}_{k_{\varphi}}(-q, q-p)=g(-q, q-p)
$$

which can be normalized with $\bar{h}_{k_{\varphi}}(0,0)=g(0,0)=1$. The propagator $\tilde{G}$ and the wave function $g(-q, q-p)$ should be optimized for a most complete elimination of terms quartic in the quark fields. Neglecting the remaining $\psi^{4}$ terms and terms of higher order in $\psi$ (e.g, $\left.\psi^{6}\right)$ one arrives at the initial value for $\Gamma_{k_{\varphi}}$. In the present paper we often do not want to keep the complete momentum dependence of $Z_{\psi, k}, Z_{\varphi, k}$ and $\bar{h}_{k}$. Useful definitions of the initial values of the parameters of the model in section 2 are then

$$
\begin{aligned}
Z_{\psi}\left(k_{\varphi}\right) & =\left.Z_{\psi, k_{\varphi}}(q)\right|_{q^{2}=0} \\
Z_{\varphi}\left(k_{\varphi}\right) & =\left.\frac{1}{2 q^{2}}\left(\tilde{G}^{-1}(q)-\tilde{G}^{-1}(0)\right)\right|_{q^{2}=k_{\varphi}^{2}} \\
\bar{m}^{2}\left(k_{\varphi}\right) & =\frac{1}{2} \tilde{G}^{-1}(0) \\
\bar{h}\left(k_{\varphi}\right) & =\left.\bar{h}_{k_{\varphi}}(-q, q)\right|_{q^{2}=0} \equiv 1
\end{aligned}
$$


Although the results of [14] should only be considered as rough estimates it seems convenient to use them as a guide for the choice of initial values of the various couplings. The values found for the transition scale $k_{\varphi}$ and the scalar mass at this scale are [14] $k_{\varphi}=630 \mathrm{MeV}, \bar{m}\left(k_{\varphi}\right)=120 \mathrm{MeV}$. The $q^{2}$-dependence of $\tilde{G}$ was not computed very reliably in ref [14]. Large- $N_{c}$ estimates use a rather weak $q^{2}$-dependence [17]. As a typical guess we consider here, somewhat arbitrarily, that $\tilde{G}^{-1}\left(q^{2}=k_{\varphi}^{2}\right)$ exceeds $\tilde{G}^{-1}\left(q^{2}=0\right)$ by $15 \%$. This leads to $Z_{\varphi}\left(k_{\varphi}\right)=0.15 \frac{\bar{m}^{2}\left(k_{\varphi}\right)}{k_{\varphi}^{2}} \simeq \frac{1}{180}$. With $Z_{\psi}\left(k_{\varphi}\right)=1, \bar{h}\left(k_{\varphi}\right)=1$ this corresponds to a large renormalized Yukawa coupling of $h^{2}\left(k_{\varphi}\right)=180$. We will see later (section 7) that for strong initial Yukawa couplings the decisive parameter is the ratio

$$
\tilde{\epsilon}_{0}=Z_{\psi}^{2}\left(k_{\varphi}\right) \frac{\bar{m}^{2}\left(k_{\varphi}\right)}{k_{\varphi}^{2}} .
$$

The values of [14] correspond to $\tilde{\epsilon}_{0}=0.036$. Both, $Z_{\psi}$ and $\bar{m}$, may be somewhat lower than the values from [14] and we will often use typical values $\bar{m} Z_{\psi}\left(k_{\varphi}\right)=89 \mathrm{MeV}(63 \mathrm{MeV})$ for which $\tilde{\epsilon}_{0} \equiv \bar{m}^{2} Z_{\psi}^{2}\left(k_{\varphi}\right) / k_{\varphi}^{2} \simeq 0.02(0.01)$ and $h^{2}\left(k_{\varphi}\right) \simeq 330(660)$ if the same assumption on the momentum dependence of $\tilde{G}^{-1}(q)$ is made as above. The dependence of our results on the choice of initial values will be discussed in detail in section 7 .

We note that the use of the identity (3.1) does not lead to anomalous $U_{A}(1)$ violating meson interactions and (3.3) conserves the axial $U_{A}(1)$ symmetry. Consequently the solution of the flow equations for the $k$-dependence of the average potential also conserves this symmetry. The formalism has therefore to be extended to incorporate anomalous fermion interactions of the type Det $\left(\bar{\psi}^{a} \psi_{b}\right)$ into the mesonic picture. This issue may be addressed for the time being by introducing a term $-\frac{1}{2} \bar{\nu} \xi$ into $U_{k}$ of (3.4) as a phenomenologically determined coupling. We leave this for future work and concentrate here on the $U_{A}(1)$ conserving case $\bar{\nu}=0$ and later (section 6) on the opposite extreme $\bar{\nu} \rightarrow \infty$ for $N=2$. We also observe that a generalization of the formalism of [14] may lead to nonvanishing meson self-interactions $\bar{\lambda}_{1}, \bar{\lambda}_{2}$ at the scale $k_{\varphi}$ as predicted by large $-N_{c}$ results [17].

At the scale $k_{\varphi}$ the effective potential (3.4) has its minimum at the origin. As a result of quantum fluctuations with momenta $q^{2}<k_{\varphi}^{2}$ one expects that the potential changes its shape and ends up at $k=0$ with a minimum for $\rho>0$, resulting in a spontaneous breaking of chiral symmetry. The aim of this paper is to derive flow equations for the $k$-dependence of $Z_{\psi}, Z_{\varphi}, U_{k}$ and $\bar{h}$ and to compute the observable quantities at $k=0$ described in the last section from the initial values (3.7). Solving the flow equations numerically we find that chiral symmetry breaking indeed occurs as demonstrated in figure 1.

We begin with the evolution equation for the effective average potential $U_{k}$. Except for the $k$-dependence of $\bar{\nu}$ the evolution equation for the potential can be obtained (c.f. appendix A) by studying a constant scalar field configuration which is real and diagonal

$$
\varphi_{a b}=\varphi_{a} \delta_{a b}=\widehat{m}_{a} \delta_{a b}
$$

We evaluate the exact evolution equation (1.1) for this configuration and insert the truncation (2.1) into the right hand side. The flow equation has a fermionic and a bosonic 


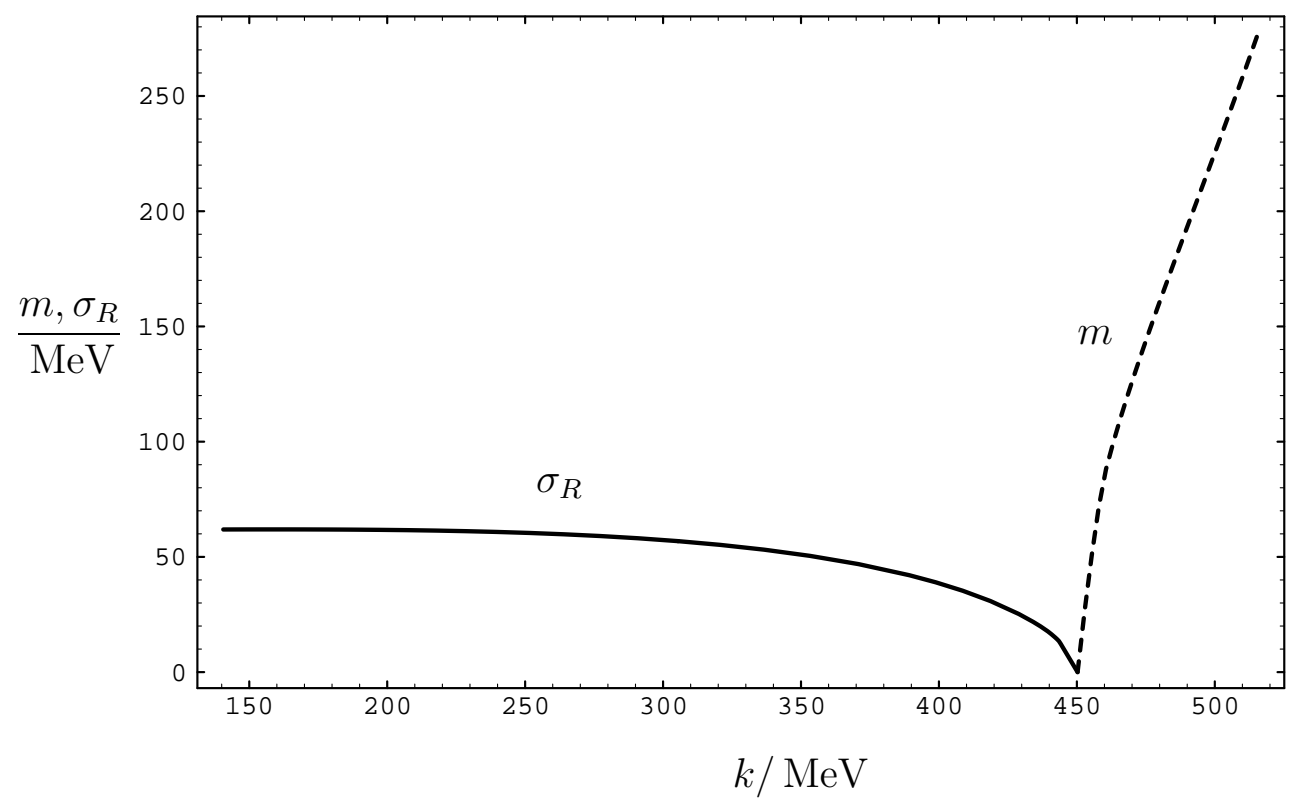

Figure 1: Evolution of the renormalized mass $m$ in the symmetric regime (dashed line) and the vacuum expectation value $\sigma_{R}$ of the scalar field in the SSB regime (solid line) as functions of $k$ for the $U_{L}(2) \times U_{R}(2)$ model. Initial values are $\lambda_{1}\left(k_{\varphi}\right)=\lambda_{2}\left(k_{\varphi}\right)=0$ for $k_{\varphi}=630 \mathrm{MeV}$ with $h^{2}\left(k_{\varphi}\right)=300$ and $\tilde{\epsilon}_{0}=0.01$.

contribution. The bosonic part follows by neglecting for a moment the quarks $[10,9]$ :

$$
\begin{aligned}
\frac{\partial}{\partial t} U_{k}= & \frac{1}{2} \int \frac{d^{d} q}{(2 \pi)^{d}} \frac{\partial}{\partial t} R_{k}(q)\left\{\sum_{a}\left[\frac{1}{Z_{\varphi} P+M_{R a}^{2}}+\frac{1}{Z_{\varphi} P+M_{I a}^{2}}\right]\right. \\
+ & \sum_{a \neq b}\left[\frac{1}{Z_{\varphi} P+\left(M_{R a b}^{+}\right)^{2}}+\frac{1}{Z_{\varphi} P+\left(M_{R a b}^{-}\right)^{2}}\right. \\
& \left.\left.+\frac{1}{Z_{\varphi} P+\left(M_{I a b}^{+}\right)^{2}}+\frac{1}{Z_{\varphi} P+\left(M_{I a b}^{-}\right)^{2}}\right]\right\} .
\end{aligned}
$$

We observe the appearance of the (massless) inverse average propagator

$$
P(q)=q^{2}+Z_{\varphi}^{-1} R_{k}(q)=\frac{q^{2}}{1-e^{-\frac{q^{2}}{k^{2}}}}
$$

which incorporates the infrared cutoff function $R_{k}(1.2)$. The dependence of the various mass eigenvalues on $\varphi_{a}$ can be found in appendix B. We restrict the discussion here to the approximation $\bar{\nu}=0$ which corresponds to neglecting the mass difference between the pseudo-scalar pion triplet and the $\eta^{\prime}$ singlet. The mass eigenvalues on the right hand side of (3.10) are then given by (B.10)-(B.15). In order to express the eigenvalues $\widehat{m}_{a}^{2}$ in terms of $\rho$ and $\tau_{2}$ we consider a particular configuration $\varphi$ where $N-1$ eigenvalues are 
equal to $\widehat{m}_{1}^{2}$ such that

$$
\begin{aligned}
\rho & =(N-1) \widehat{m}_{1}^{2}+\hat{m}_{N}^{2} \\
\operatorname{tr} \phi^{2} & =\frac{N-1}{N}\left(\widehat{m}_{1}^{2}-\widehat{m}_{N}^{2}\right)^{2}, \quad \phi=\varphi^{\dagger} \varphi-\frac{1}{N} \rho
\end{aligned}
$$

or

$$
\begin{aligned}
\varphi_{1}^{2} & =\widehat{m}_{1}^{2}=\frac{1}{N}\left(\rho+\sqrt{\tau_{2}}\right) \\
\varphi_{N}^{2} & =\widehat{m}_{N}^{2}=\frac{1}{N}\left(\rho-(N-1) \sqrt{\tau_{2}}\right) .
\end{aligned}
$$

This defines the right hand side of the evolution equation (3.10) as a function of $\rho$ and $\tau_{2}$. In the symmetric regime the evolution of the couplings $\bar{\lambda}_{1}, \bar{\lambda}_{2}$ and the mass term $\bar{m}^{2}$ can now be extracted by suitable differentiation of eq. (3.10) with respect to $\rho$ and $\tau_{2}$, evaluated for $\rho=\tau_{2}=0$. One finds for the bosonic contributions

$$
\begin{aligned}
\frac{\partial}{\partial t} \bar{m}^{2} & =-\frac{1}{2} \int \frac{d^{d} q}{(2 \pi)^{d}} \frac{\partial R_{k}}{\partial t} \frac{2\left(N^{2}+1\right) \bar{\lambda}_{1}+\left(N^{2}-1\right) \bar{\lambda}_{2}}{\left(Z_{\varphi} P+\bar{m}^{2}\right)^{2}} \\
\frac{\partial}{\partial t} \bar{\lambda}_{1} & =\int \frac{d^{d} q}{(2 \pi)^{d}} \frac{\partial R_{k}}{\partial t} \frac{2\left(N^{2}+4\right) \bar{\lambda}_{1}^{2}+2\left(N^{2}-1\right) \bar{\lambda}_{1} \bar{\lambda}_{2}+\left(N^{2}-1\right) \bar{\lambda}_{2}^{2}}{\left(Z_{\varphi} P+\bar{m}^{2}\right)^{3}} \\
\frac{\partial}{\partial t} \bar{\lambda}_{2} & =\int \frac{d^{d} q}{(2 \pi)^{d}} \frac{\partial R_{k}}{\partial t} \frac{12 \bar{\lambda}_{1} \bar{\lambda}_{2}+2\left(N^{2}-3\right) \bar{\lambda}_{2}^{2}}{\left(Z_{\varphi} P+\bar{m}^{2}\right)^{3}}
\end{aligned}
$$

If in the course of the evolution towards smaller values of $k$ the mass term $\bar{m}^{2}$ becomes negative we should switch to the couplings appropriate to the regime with spontaneous symmetry breaking (SSB regime). There we define

$$
\begin{aligned}
& \bar{\lambda}_{1}=U_{k}^{\prime \prime}\left(\rho_{0}, \tau_{2}=0\right) \\
& \bar{\lambda}_{2}=\frac{4}{N-1} \frac{\partial U_{k}}{\partial \tau_{2}}\left(\rho_{0}, \tau_{2}=0\right)
\end{aligned}
$$

where $\rho_{0}$ corresponds to the $k$-dependent minimum of the potential. We use that

$$
U_{k}^{\prime}\left(\rho_{0}\right)=0
$$

is valid for all $k$ and therefore obtain

$$
\frac{\partial}{\partial t} \rho_{0}=-\frac{1}{\bar{\lambda}_{1}} \frac{\partial}{\partial t} U_{k}^{\prime}\left(\rho_{0}\right)
$$

The evolution equations for $\rho_{0}, \bar{\lambda}_{1}$ and $\bar{\lambda}_{2}$ follow directly from the definitions $(3.17),(3.19)$. For $\bar{\nu}=0$ they read

$$
\frac{\partial}{\partial t} \rho_{0}=\frac{1}{2} \int \frac{d^{d} q}{(2 \pi)^{d}} \frac{\partial R_{k}}{\partial t}\left\{\frac{N^{2}}{\left(Z_{\varphi} P\right)^{2}}+\frac{3}{\left(Z_{\varphi} P+2 \bar{\lambda}_{1} \rho_{0}\right)^{2}}+\frac{\left(N^{2}-1\right)\left(1+\frac{\bar{\lambda}_{2}}{\bar{\lambda}_{1}}\right)}{\left(Z_{\varphi} P+\bar{\lambda}_{2} \rho_{0}\right)^{2}}\right\}
$$




$$
\begin{aligned}
\frac{\partial}{\partial t} \bar{\lambda}_{1}= & \int \frac{d^{d} q}{(2 \pi)^{d}} \frac{\partial R_{k}}{\partial t}\left\{\frac{N^{2} \bar{\lambda}_{1}^{2}}{\left(Z_{\varphi} P\right)^{3}}+\frac{9 \bar{\lambda}_{1}^{2}}{\left(Z_{\varphi} P+2 \bar{\lambda}_{1} \rho_{0}\right)^{3}}+\frac{\left(N^{2}-1\right)\left(\bar{\lambda}_{1}+\bar{\lambda}_{2}\right)^{2}}{\left(Z_{\varphi} P+\bar{\lambda}_{2} \rho_{0}\right)^{3}}\right\} \\
\frac{\partial}{\partial t} \bar{\lambda}_{2} & =\int \frac{d^{d} q}{(2 \pi)^{d}} \frac{\partial R_{k}}{\partial t}\left\{\frac{N^{2}}{4} \frac{\bar{\lambda}_{2}^{2}}{\left(Z_{\varphi} P\right)^{3}}\right. \\
& +\frac{9\left(N^{2}-4\right)}{4} \frac{\bar{\lambda}_{2}^{2}}{\left(Z_{\varphi} P+\bar{\lambda}_{2} \rho_{0}\right)^{3}}+\frac{N^{2} \bar{\lambda}_{2}}{4 \rho_{0}}\left[\frac{1}{\left(Z_{\varphi} P+\bar{\lambda}_{2} \rho_{0}\right)^{2}}-\frac{1}{\left(Z_{\varphi} P\right)^{2}}\right] \\
& \left.+\frac{3 \bar{\lambda}_{2}\left(\frac{1}{4} \bar{\lambda}_{2}+\bar{\lambda}_{1}\right)}{\rho_{0}\left(\frac{1}{2} \bar{\lambda}_{2}-\bar{\lambda}_{1}\right)}\left[\frac{1}{\left(Z_{\varphi} P+2 \bar{\lambda}_{1} \rho_{0}\right)^{2}}-\frac{1}{\left(Z_{\varphi} P+\bar{\lambda}_{2} \rho_{0}\right)^{2}}\right]\right\} .
\end{aligned}
$$

It is straightforward to check that in the limits $\bar{m}^{2} \rightarrow 0, \rho_{0} \rightarrow 0$ the flow equations for $\bar{\lambda}_{1}$, $\bar{\lambda}_{2}$ coincide in the symmetric and SSB regime. We also note that the evolution equation for $\bar{\lambda}_{2}$ depends on the precise definition of this coupling. This issue is shortly addressed in appendix $\mathrm{C}$ where we also give an alternative formulation of eq. (3.22).

Next we turn to the fermionic contribution to the evolution equation for the effective average potential which we denote by $\partial U_{k F} / \partial t$. Using the general formulae of [22] it can be computed without additional effort for the extended ansatz (3.3) where we keep the momentum dependence of $Z_{\psi, k}$ and part of the momentum dependence of the Yukawa coupling with $\bar{h}_{k}(q) \equiv \bar{h}(-q, q)$. With $P_{F}$ given in appendix D and setting for a moment $Z_{\psi, k}(q)=1$ one obtains:

$$
\frac{\partial}{\partial t} U_{k F}=-2^{\frac{d}{2}-1} N_{c} \int \frac{d^{d} q}{(2 \pi)^{d}} \sum_{a=1}^{N} \frac{\partial P_{F}(q)}{\partial t}\left(P_{F}(q)+m_{a}^{2}(q)\right)^{-1} .
$$

Here $m_{a}^{2}(q)$ are the $N$ real nonnegative eigenvalues of the $N \times N$ matrix $\bar{h}_{k}(q) \bar{h}_{k}^{*}(q) \varphi^{\dagger} \varphi$ with momentum dependent Yukawa couplings defined by (A.8). Here we have taken into account the $N_{c}$ colors of the quarks. For a given value of $q$ we may use the identity (with $\partial / \partial t$ acting only on $P_{F}, m^{2}=\operatorname{diag}\left(m_{a}^{2}\right)$ and tr taken in flavor space)

$$
\begin{aligned}
\sum_{a=1}^{N} \frac{\partial P_{F}}{\partial t}\left(P_{F}+m_{a}^{2}\right)^{-1} & =\frac{\partial}{\partial t} \operatorname{tr} \ln \left(P_{F}+m^{2}\right)=\frac{\partial}{\partial t} \ln \operatorname{det}\left(P_{F}+m^{2}\right) \\
& =\frac{\partial}{\partial t} \ln \operatorname{det}\left(P_{F}+\left|\bar{h}_{k}(q)\right|^{2} \varphi^{\dagger} \varphi\right) \\
& =\frac{\partial P_{F}}{\partial t} \operatorname{tr}\left(P_{F}+\left|\bar{h}_{k}(q)\right|^{2} \varphi^{\dagger} \varphi\right)^{-1} .
\end{aligned}
$$

This gives an expression for general $\varphi$

$$
\frac{\partial}{\partial t} U_{k F}=-2^{\frac{d}{2}-1} N_{c} \int \frac{d^{d} q}{(2 \pi)^{d}} \frac{\partial P_{F}}{\partial t} \operatorname{tr}\left(P_{F}+\left|\bar{h}_{k}(q)\right|^{2} \varphi^{\dagger} \varphi\right)^{-1} .
$$


Using the particular configuration with $N-1$ equal eigenvalues and the relation (3.13) one finally obtains

$$
\begin{aligned}
\frac{\partial}{\partial t} U_{k F} & =-2^{\frac{d}{2}-1} N_{c} \int \frac{d^{d} q}{(2 \pi)^{d}} \frac{\partial P_{F}}{\partial t}\left\{(N-1)\left(P_{F}+\frac{1}{N}\left|\bar{h}_{k}\right|^{2}\left(\rho+\sqrt{\tau_{2}}\right)\right)^{-1}\right. \\
& \left.+\left(P_{F}+\frac{1}{N}\left|\bar{h}_{k}\right|^{2}\left(\rho-(N-1) \sqrt{\tau_{2}}\right)\right)^{-1}\right\} .
\end{aligned}
$$

We emphasize that the Yukawa couplings in (3.3) conserve the axial $U_{A}(1)$ symmetry. The fermionic contribution to $\partial U_{k F} / \partial t$ is therefore independent of $\xi$. The fermionic wave function renormalization $Z_{\psi, k}(q)$ is easily restored if we replace the function $P_{F}(q)$ in $(3.26)$ by $Z_{\psi, k}^{2}(q) P_{F}(q)$ and note that the partial derivative $\frac{\widehat{\partial}}{\partial t}\left(Z_{\psi, k}^{2} P_{F}\right)$ only acts on the pieces related to the infrared cutoff $R_{k}$. Within the truncation (3.3) the fermionic contribution to the evolution equation (3.25) is then exact. Eq. (3.26) gives the exact result for $N=2$ whereas for $N>2$ one has an additional dependence on invariants $\tau_{i}, i \geq 3$, defined in appendix B, which can be extracted from (3.25). The derivatives of $\frac{\partial}{\partial t} U_{k F}$ with respect to $\rho$ and $\tau_{2}$ can be written in a suggestive form as

$$
\begin{aligned}
\frac{\partial}{\partial t} U_{k F}^{\prime} & =-2^{\frac{d}{2}-1} \frac{N_{c}}{N} \int \frac{d^{d} q}{(2 \pi)^{d}}\left|\bar{h}_{k}\right|^{2} \\
& \times \frac{\widehat{\partial}}{\partial t}\left\{\frac{N-1}{Z_{\psi, k}^{2} P_{F}+\frac{1}{N}\left|\bar{h}_{k}\right|^{2}\left(\rho+\sqrt{\tau_{2}}\right)}+\frac{1}{Z_{\psi, k}^{2} P_{F}+\frac{1}{N}\left|\bar{h}_{k}\right|^{2}\left(\rho-(N-1) \sqrt{\tau_{2}}\right)}\right\} \\
\frac{\partial}{\partial t} \frac{\partial U_{k F}}{\partial \tau_{2}} & =-2^{\frac{d}{2}-2} N_{c} \frac{N-1}{N} \frac{1}{\sqrt{\tau_{2}}} \int \frac{d^{d} q}{(2 \pi)^{d}}\left|\bar{h}_{k}\right|^{2} \\
& \times \frac{1}{\partial t}\left\{\frac{1}{Z_{\psi, k}^{2} P_{F}+\frac{1}{N}\left|\bar{h}_{k}\right|^{2}\left(\rho+\sqrt{\tau_{2}}\right)}-\frac{1}{Z_{\psi, k}^{2} P_{F}+\frac{1}{N}\left|\bar{h}_{k}\right|^{2}\left(\rho-(N-1) \sqrt{\tau_{2}}\right)}\right\}
\end{aligned}
$$

with the formal definition (cf. appendix D)

$$
\frac{\widehat{\partial}}{\partial t} \equiv \frac{1}{Z_{\varphi, k}} \frac{\partial R_{k}}{\partial t} \frac{\partial}{\partial P}+\frac{2}{Z_{\psi, k}} \frac{P_{F}}{1+r_{F}} \frac{\partial\left[Z_{\psi, k} r_{F}\right]}{\partial t} \frac{\partial}{\partial P_{F}} .
$$

We may now combine the bosonic and fermionic contributions to the running of the renormalized couplings. Here we restrict the discussion again to momentum independent $Z_{\varphi}, Z_{\psi}$ and (real) $\bar{h}$, i.e. we replace similarly to $(3.7) Z_{\varphi, k}(q) \rightarrow Z_{\varphi}(k), Z_{\psi, k}(q) \rightarrow Z_{\psi}(k)$ and $\bar{h}_{k}(q) \rightarrow \bar{h}(k)=\bar{h}^{*}(k)$. For arbitrary $d$ it is convenient to introduce dimensionless couplings in analogy to $(2.8)-(2.10)$ :

$$
\begin{aligned}
h^{2} & =Z_{\varphi}^{-1} Z_{\psi}^{-2} k^{d-4} \bar{h}^{2} \\
\lambda_{1,2} & =Z_{\varphi}^{-2} k^{d-4} \bar{\lambda}_{1,2} \\
\kappa & =k^{2-d} \rho_{R}=Z_{\varphi} k^{2-d} \rho_{0} \\
\epsilon & =k^{-2} m^{2}=Z_{\varphi}^{-1} k^{-2} \bar{m}^{2}
\end{aligned}
$$


and to define the anomalous dimensions for the scalar field, $\eta_{\varphi}$, and the fermion field, $\eta_{\psi}$, by

$$
\eta_{\varphi}=-\frac{\partial}{\partial t} \ln Z_{\varphi, k}, \quad \eta_{\psi}=-\frac{\partial}{\partial t} \ln Z_{\psi, k}
$$

We also use dimensionless integrals

$$
\begin{aligned}
l_{n}^{d}\left(w ; \eta_{\varphi}\right) & =l_{n}^{d}(w)-\eta_{\varphi} \hat{l}_{n}^{d}(w) \\
& =\frac{n}{4} v_{d}^{-1} k^{2 n-d} \int \frac{d^{d} q}{(2 \pi)^{d}}\left(\frac{1}{Z_{\varphi}} \frac{\partial R_{k}(q)}{\partial t}\right)\left(P+w k^{2}\right)^{-(n+1)} \\
& =-\frac{1}{2} k^{2 n-d} \int_{0}^{\infty} d x x^{\frac{d}{2}-1} \frac{\widehat{\partial}}{\partial t}\left(P+w k^{2}\right)^{-n} \\
l_{n_{1}, n_{2}}^{d}\left(w_{1}, w_{2} ; \eta_{\varphi}\right) & =l_{n_{1}, n_{2}}^{d}\left(w_{1}, w_{2}\right)-\eta_{\varphi} \hat{l}_{n_{1}, n_{2}}^{d}\left(w_{1}, w_{2}\right) \\
& =-\frac{1}{2} k^{2\left(n_{1}+n_{2}\right)-d} \int_{0}^{\infty} d x x^{\frac{d}{2}-1} \frac{\widehat{\partial}}{\partial t}\left\{\left(P+w_{1} k^{2}\right)^{-n_{1}}\left(P+w_{2} k^{2}\right)^{-n_{2}}\right\}
\end{aligned}
$$

where the part $\sim \eta_{\varphi} \hat{l}_{n}^{d}(w)$ arises from the $t$-derivative acting on $Z_{\varphi}$ within $R_{k}$ (cf. (1.2)) and

$$
v_{d}^{-1}=2^{d+1} \pi^{\frac{d}{2}} \Gamma\left(\frac{d}{2}\right)
$$

The "fermionic integrals" $l_{n}^{(F) d}\left(w ; \eta_{\psi}\right)=l_{n}^{(F) d}(w)-\eta_{\psi} \breve{l}_{n}^{(F) d}(w)$ are defined analogously, with $P$ replaced by $P_{F}$. Combining (3.14) - (3.16) with (3.27), (3.28) we obtain the evolution equations for the symmetric regime:

$$
\begin{aligned}
\frac{\partial \epsilon}{\partial t} & =-\left(2-\eta_{\varphi}\right) \epsilon-2 v_{d}\left\{\left[2\left(N^{2}+1\right) \lambda_{1}+\left(N^{2}-1\right) \lambda_{2}\right] l_{1}^{d}\left(\epsilon ; \eta_{\varphi}\right)\right. \\
& \left.-2^{\frac{d}{2}} N_{c} h^{2} l_{1}^{(F) d}\left(\eta_{\psi}\right)\right\} \\
\frac{\partial \lambda_{1}}{\partial t} & =\left(d-4+2 \eta_{\varphi}\right) \lambda_{1}+2 v_{d}\left\{\left[2\left(N^{2}+4\right) \lambda_{1}^{2}+\left(N^{2}-1\right) \lambda_{2}\left(2 \lambda_{1}+\lambda_{2}\right)\right] l_{2}^{d}\left(\epsilon ; \eta_{\varphi}\right)\right. \\
& \left.-2^{\frac{d}{2}} \frac{N_{c}}{N} h^{4} l_{2}^{(F) d}\left(\eta_{\psi}\right)\right\} \\
\frac{\partial \lambda_{2}}{\partial t} & =\left(d-4+2 \eta_{\varphi}\right) \lambda_{2}+2 v_{d}\left\{\left[12 \lambda_{1} \lambda_{2}+2\left(N^{2}-3\right) \lambda_{2}^{2}\right] l_{2}^{d}\left(\epsilon ; \eta_{\varphi}\right)\right. \\
& \left.-2^{\frac{d}{2}+1} \frac{N_{c}}{N} h^{4} l_{2}^{(F) d}\left(\eta_{\psi}\right)\right\} .
\end{aligned}
$$

Similarly, the evolution equations for the SSB regime read

$$
\begin{aligned}
\frac{\partial \kappa}{\partial t} & =\left(2-d-\eta_{\varphi}\right) \kappa+2 v_{d}\left\{N^{2} l_{1}^{d}\left(\eta_{\varphi}\right)+3 l_{1}^{d}\left(2 \lambda_{1} \kappa ; \eta_{\varphi}\right)\right. \\
& \left.+\left(N^{2}-1\right)\left[1+\frac{\lambda_{2}}{\lambda_{1}}\right] l_{1}^{d}\left(\lambda_{2} \kappa ; \eta_{\varphi}\right)-2^{\frac{d}{2}} N_{c} \frac{h^{2}}{\lambda_{1}} l_{1}^{(F) d}\left(\frac{1}{N} h^{2} \kappa ; \eta_{\psi}\right)\right\}
\end{aligned}
$$




$$
\begin{aligned}
\frac{\partial \lambda_{1}}{\partial t} & =\left(d-4+2 \eta_{\varphi}\right) \lambda_{1}+2 v_{d}\left\{N^{2} \lambda_{1}^{2} l_{2}^{d}\left(\eta_{\varphi}\right)+9 \lambda_{1}^{2} l_{2}^{d}\left(2 \lambda_{1} \kappa ; \eta_{\varphi}\right)\right. \\
& \left.+\left(N^{2}-1\right)\left[\lambda_{1}+\lambda_{2}\right]^{2} l_{2}^{d}\left(\lambda_{2} \kappa ; \eta_{\varphi}\right)-2^{\frac{d}{2}} \frac{N_{c}}{N} h^{4} l_{2}^{(F) d}\left(\frac{1}{N} h^{2} \kappa ; \eta_{\psi}\right)\right\} \\
\frac{\partial \lambda_{2}}{\partial t} & =\left(d-4+2 \eta_{\varphi}\right) \lambda_{2}+2 v_{d}\left\{\frac{N^{2}}{4} \lambda_{2}^{2} l_{2}^{d}\left(\eta_{\varphi}\right)+\frac{9}{4}\left(N^{2}-4\right) \lambda_{2}^{2} l_{2}^{d}\left(\lambda_{2} \kappa ; \eta_{\varphi}\right)\right. \\
& -\frac{1}{2} N^{2} \lambda_{2}^{2} l_{1,1}^{d}\left(0, \lambda_{2} \kappa ; \eta_{\varphi}\right)+3\left[\lambda_{2}+4 \lambda_{1}\right] \lambda_{2} l_{1,1}^{d}\left(2 \lambda_{1} \kappa, \lambda_{2} \kappa ; \eta_{\varphi}\right) \\
& \left.-2^{\frac{d}{2}+1} \frac{N_{c}}{N} h^{4} l_{2}^{(F) d}\left(\frac{1}{N} h^{2} \kappa ; \eta_{\psi}\right)\right\}
\end{aligned}
$$

where we have defined

$$
l_{n}^{d}\left(\eta_{\varphi}\right) \equiv l_{n}^{d}\left(0 ; \eta_{\varphi}\right), \quad l_{n}^{(F) d}\left(\eta_{\psi}\right) \equiv l_{n}^{(F) d}\left(0 ; \eta_{\psi}\right) .
$$

No explicit dependence on $k$ appears in this scaling form of the flow equations. In the limit $\epsilon, \kappa \rightarrow 0$ one recovers for both regimes to leading order in the coupling constants the known $[16,17]$ perturbative one-loop beta functions for $\lambda_{1}$ and $\lambda_{2}$.

The system of flow equations (3.34)-(3.39) is the central piece of this work. For the quark-meson model $(d=4)$ we fix the initial conditions at $k=k_{\varphi}(t=0)$ with $\lambda_{1}\left(k_{\varphi}\right)=\lambda_{2}\left(k_{\varphi}\right)=0, \epsilon\left(k_{\varphi}\right)=Z_{\varphi}^{-1}\left(k_{\varphi}\right) k_{\varphi}^{-2} \bar{m}^{2}$. The solution of the evolution equations should then reveal the phenomenon of spontaneous chiral symmetry breaking for $k \rightarrow 0$ $(t \rightarrow-\infty)$. More precisely, we expect for some scale $k_{s}>0$ that the mass term vanishes, i.e. $\epsilon\left(k_{s}\right)=0$. Subsequently, for $k<k_{s}$ we follow the evolution of the minimum of the potential using the system $(3.37)-(3.39)$, with initial condition $\kappa\left(k_{s}\right)=0$. (The couplings $\lambda_{1}$ and $\lambda_{2}$ are continuous at $k_{s}$.) For sufficiently small values of $k$ the minimum of the potential will not move anymore, i.e.

$$
\begin{aligned}
\lim _{k \rightarrow 0} Z_{\varphi}(k) & =Z_{\varphi} \\
\lim _{k \rightarrow 0} \rho_{R}(k) & =\lim _{k \rightarrow 0} Z_{\varphi}(k) \rho_{0}(k)=\rho_{R}=Z_{\varphi} \rho_{0} \\
& =N \sigma_{R}^{2}=N Z_{\varphi} \sigma_{0}^{2}=\frac{N}{4} f_{\pi}^{2} \\
\lim _{k \rightarrow 0} \kappa(k) & \rightarrow \rho_{R} k^{-2} .
\end{aligned}
$$

Supplementing the flow equations for $\kappa, \lambda_{1}$ and $\lambda_{2}$ by the one for the Yukawa coupling $h^{2}$ and inserting the anomalous dimensions - these quantities will be computed in the next two sections - we can now study how the shape of the average potential changes as $k$ is lowered. We have integrated the flow equations numerically for $d=4$ and $N_{c}=3$ from $t_{i}=0$ corresponding to $k=k_{\varphi}$ to $t_{f}=\ln \left(m_{\pi} / k_{\varphi}\right)$. This endpoint of the numerical integration simulates the pion mass threshold which is neglected in our approximation of vanishing quark masses. We ignore here all dependence of the threshold functions on the anomalous dimensions $\eta_{\varphi}$ and $\eta_{\psi}$. This approximations will be justified in section 7 . 


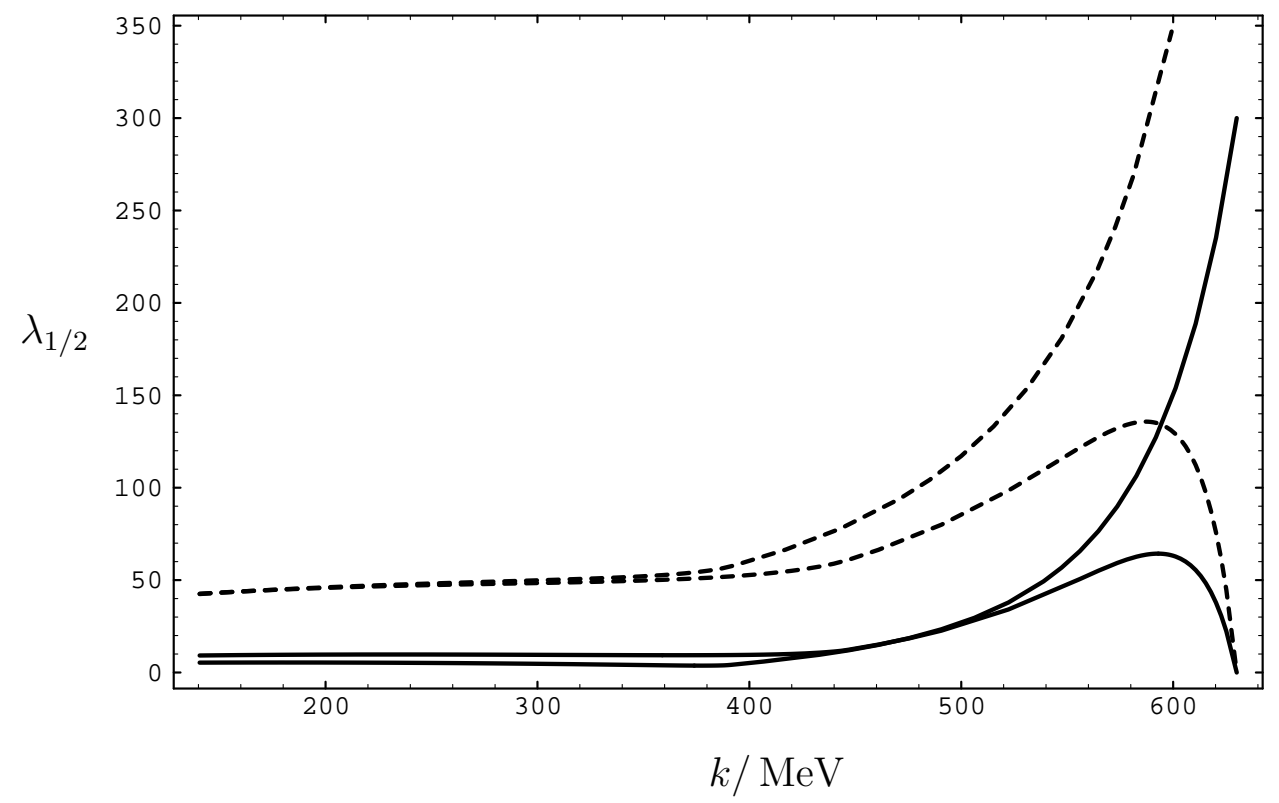

Figure 2: Flow of $\lambda_{1}$ (solid lines) and $\lambda_{2}$ (dashed lines) with $k$, for the $U_{L}(2) \times U_{R}(2)$ model with two sets of initial conditions at $k_{\varphi}=630 \mathrm{MeV}$ with $h^{2}\left(k_{\varphi}\right)=300$ and $\tilde{\epsilon}_{0}=0.01$.

We use first $k_{\varphi}=630 \mathrm{MeV}, \tilde{\epsilon}_{0}=0.01$ and $Z_{\psi}\left(k_{\varphi}\right)=1, h^{2}\left(k_{\varphi}\right)=Z_{\varphi}^{-1}\left(k_{\varphi}\right)=300$. For the initial values of $\lambda_{1}$ and $\lambda_{2}$ we employ two different sets of boundary conditions. One corresponds to the approximations used in [14]

$$
\lambda_{1}\left(k_{\varphi}\right)=\lambda_{2}\left(k_{\varphi}\right)=0
$$

The other set is obtained in the large $-N_{c}$ limit of the $U_{L}(N) \times U_{R}(N)$ model [17] and reads

$$
\begin{aligned}
\lambda_{1}\left(k_{\varphi}\right) & =\frac{2}{N} h^{2}\left(k_{\varphi}\right) \\
\lambda_{2}\left(k_{\varphi}\right) & =\frac{4}{N} h^{2}\left(k_{\varphi}\right) .
\end{aligned}
$$

For the numerical investigations we will concentrate in the present work on $N=2$. In fig. 1 we have plotted the renormalized mass $m$ as a function of $k$. Starting from a very large value $m\left(k_{\varphi}\right)=1091 \mathrm{MeV}$ the mass rapidly decreases and reaches zero for $k_{s} \simeq 450 \mathrm{MeV}$. For $k<k_{s}$ the minimum of the potential occurs for $\rho_{0}>0$ and we have to use the flow equations for the SSB regime. In fig. 1 we also show the $k$-dependence of the location of the minimum, $\sigma_{R}(k)=\left(\rho_{R}(k) / N\right)^{\frac{1}{2}}$, and see how it stabilizes for $k \lesssim 250 \mathrm{MeV}$. The final result for $\sigma_{R}$ is rather insensitive to the exact choice of the endpoint $k_{f}=m_{\pi}$. In fig. 2 we display the $k$-dependence of the quartic couplings $\lambda_{1}$ and $\lambda_{2}$ for the two sets of boundary conditions. We observe that the result for $k=k_{f}$ does not depend strongly on the initial values. This is a first manifestation of the infrared stability mentioned in 
the introduction. We will explain the origin of this behavior in more detail later (section 7), since for an understanding we first need to discuss the anomalous dimensions and the running of $h^{2}$.

\section{Scalar anomalous dimension}

A computation of $f_{\pi}$ in terms of the four-quark interaction at the scale $k_{\varphi}$ requires the ratio $Z_{\varphi}(0) / Z_{\varphi}\left(k_{\varphi}\right)$. An evaluation of this ratio is the subject of this section. We will begin with the determination of the flow equation for the momentum dependent scalar wave function renormalization $Z_{\varphi, k}(q)$ or, equivalently, the scalar anomalous dimension $\eta_{\varphi, k}(q) \equiv-Z_{\varphi, k}^{-1}(q) \partial_{t} Z_{\varphi, k}(q)$. For this purpose we have to consider a spatially varying scalar field configuration. We choose a nondiagonal distortion of the constant vacuum configuration (2.3):

$$
\varphi_{a b}(x)=\varphi \delta_{a b}+\left[\delta \varphi e^{-i Q x}+\delta \varphi^{*} e^{i Q x}\right] \Sigma_{a b}=\varphi_{a b}^{*}(x)
$$

with

$$
\Sigma_{a b}=\delta_{a 1} \delta_{b 2}-\delta_{a 2} \delta_{b 1}
$$

or, in momentum space (with $\delta\left(q_{1}, q_{2}\right)=(2 \pi)^{d} \delta\left(q_{1}-q_{2}\right)$ ),

$$
\begin{aligned}
\varphi_{a b}(q) & =\varphi \delta(q, 0) \delta_{a b}+\left[\delta \varphi \delta(q, Q)+\delta \varphi^{*} \delta(q,-Q)\right] \Sigma_{a b} \\
& \equiv \varphi \delta(q, 0) \delta_{a b}+\Delta(q, Q) \Sigma_{a b}
\end{aligned}
$$

Expanding around $\varphi$ at the potential minimum, we observe that $\delta \varphi$ corresponds to an excitation of the massless charged pion $\pi^{ \pm}$. We keep the discussion here for general $\varphi$. If we supplement the scalar configuration by a fermionic background

$$
\psi_{\alpha}=\bar{\psi}_{\alpha}=0
$$

$\Gamma_{k}^{(2)}$ is easily seen to be block-diagonal. It decays into matrices acting in scalar and fermion subspaces, $\Gamma_{S k}^{(2)}$ and $\Gamma_{F k}^{(2)}$, respectively. Hence, we can read off from (1.1)

$$
\begin{aligned}
\frac{\partial}{\partial t} Z_{\varphi, k}(Q) & =\frac{1}{4 Q^{2}}\left(\operatorname { l i m } _ { \delta \varphi , \delta \varphi ^ { * } \rightarrow 0 } \frac { \partial } { \partial ( \delta \varphi \delta \varphi ^ { * } ) } \left\{\frac{1}{2} \operatorname{Tr}\left[\left(\Gamma_{S k}^{(2)}+R_{k}\right)^{-1} \frac{\partial R_{k}}{\partial t}\right]\right.\right. \\
& \left.\left.-\operatorname{Tr}\left[\left(\Gamma_{F k}^{(2)}+R_{F k}\right)^{-1} \frac{\partial R_{F k}}{\partial t}\right]\right\}-(Q \rightarrow 0)\right)
\end{aligned}
$$

The right hand side may be evaluated by expanding the traces in powers of $\delta \varphi$ and $\delta \varphi^{*}$ up to order $\delta \varphi \delta \varphi^{*}$ and subtracting all $Q$-independent terms. The flow equations for the renormalization constant $Z_{\varphi}(k)$ or equivalently $\eta_{\varphi}(k)$ are now determined as

$$
\frac{\partial}{\partial t} Z_{\varphi}(k)=\lim _{Q^{2} \rightarrow 0} \frac{\partial}{\partial t} Z_{\varphi, k}(Q), \quad \eta_{\varphi}(k)=\lim _{Q^{2} \rightarrow 0} \eta_{\varphi, k}(Q)=-\frac{\partial}{\partial t} \ln Z_{\varphi}(k) .
$$


In order to evaluate the right hand side we split

$$
\Gamma_{k}^{(2)}=\Gamma_{k, 0}^{(2)}+\Delta \Gamma_{k}^{(2)}
$$

such that all $\delta \varphi, \delta \varphi^{*}$ dependence is contained in $\Delta \Gamma_{k}^{(2)}$. We may then expand

$$
\begin{aligned}
\operatorname{Tr}\left[\left(\Gamma_{k}^{(2)}+R_{k}\right)^{-1} \frac{\partial R_{k}}{\partial t}\right] & =\operatorname{Tr}\left[\left(\Gamma_{k, 0}^{(2)}+R_{k}\right)^{-1} \frac{\partial R_{k}}{\partial t}\right] \\
& +\operatorname{Tr}\left[\frac{\partial}{\partial t}\left\{\left(\Gamma_{k, 0}^{(2)}+R_{k}\right)^{-1} \Delta \Gamma_{k}^{(2)}\right\}\right] \\
& -\frac{1}{2} \operatorname{Tr}\left[\frac{\partial}{\partial t}\left\{\left(\Gamma_{k, 0}^{(2)}+R_{k}\right)^{-1} \Delta \Gamma_{k}^{(2)}\left(\Gamma_{k, 0}^{(2)}+R_{k}\right)^{-1} \Delta \Gamma_{k}^{(2)}\right\}\right] \\
& +\mathcal{O}\left(\Delta^{3}\right)
\end{aligned}
$$

and compute the right hand side of (4.5) from the first terms. Details of the calculation can be found in appendix E. Taking the limit $Q^{2} \rightarrow 0$ and neglecting all momentum dependence of the Yukawa coupling and wave function renormalizations we find for the SSB regime

$$
\begin{aligned}
\eta_{\varphi} & =8 \frac{v_{d}}{d} \kappa\left\{2 \lambda_{1}^{2} m_{2,2}^{d}\left(0,2 \kappa \lambda_{1} ; \eta_{\varphi}\right)+\frac{N^{2}-2}{4} \lambda_{2}^{2} m_{2,2}^{d}\left(0, \kappa \lambda_{2} ; \eta_{\varphi}\right)\right\} \\
& +2^{\frac{d}{2}+2} \frac{v_{d}}{d} N_{c} h^{2} m_{4}^{(F) d}\left(\frac{1}{N} \kappa h^{2} ; \eta_{\psi}\right)
\end{aligned}
$$

and for the symmetric regime

$$
\eta_{\varphi}=2^{\frac{d}{2}+2} \frac{v_{d}}{d} N_{c} h^{2} m_{4}^{(F) d}\left(0 ; \eta_{\psi}\right) .
$$

Here we have defined the threshold functions

$$
\begin{aligned}
m_{n_{1}, n_{2}}^{d}\left(w_{1}, w_{2} ; \eta_{\varphi}\right) & \equiv m_{n_{1}, n_{2}}^{d}\left(w_{1}, w_{2}\right)-\eta_{\varphi} \hat{m}_{n_{1}, n_{2}}^{d}\left(w_{1}, w_{2}\right) \\
& =-\frac{1}{2} k^{2\left(n_{1}+n_{2}-1\right)-d} \int_{0}^{\infty} d x x^{\frac{d}{2}} \frac{\widehat{\partial}}{\partial t}\left\{\frac{\dot{P}(x)}{\left[P(x)+k^{2} w_{1}\right]^{n_{1}}} \frac{\dot{P}(x)}{\left[P(x)+k^{2} w_{2}\right]^{n_{2}}}\right\}
\end{aligned}
$$

and

$$
\begin{aligned}
m_{4}^{(F) d}\left(w ; \eta_{\psi}\right) & =m_{4}^{(F) d}(w)-\eta_{\psi} \check{m}_{4}^{(F) d}(w) \\
& =-\frac{1}{2} k^{4-d} \int_{0}^{\infty} d x x^{\frac{d}{2}+1} \frac{\widehat{\partial}}{\partial t}\left(\frac{\partial}{\partial x} \frac{1+r_{F}(x)}{P_{F}(x)+k^{2} w}\right)^{2}
\end{aligned}
$$

where

$$
x=q^{2}, \quad P(x) \equiv P(q), \quad \dot{P}(x) \equiv \frac{\partial}{\partial x} P(x), \quad \frac{\widehat{\partial}}{\partial t} \dot{P} \equiv \frac{\partial}{\partial x} \frac{\widehat{\partial}}{\partial t} P .
$$

From the anomalous dimension $\eta_{\varphi}(k)$ the wave function renormalization can be obtained by numerical integration of (3.31). We have plotted the result in fig. 3 for 


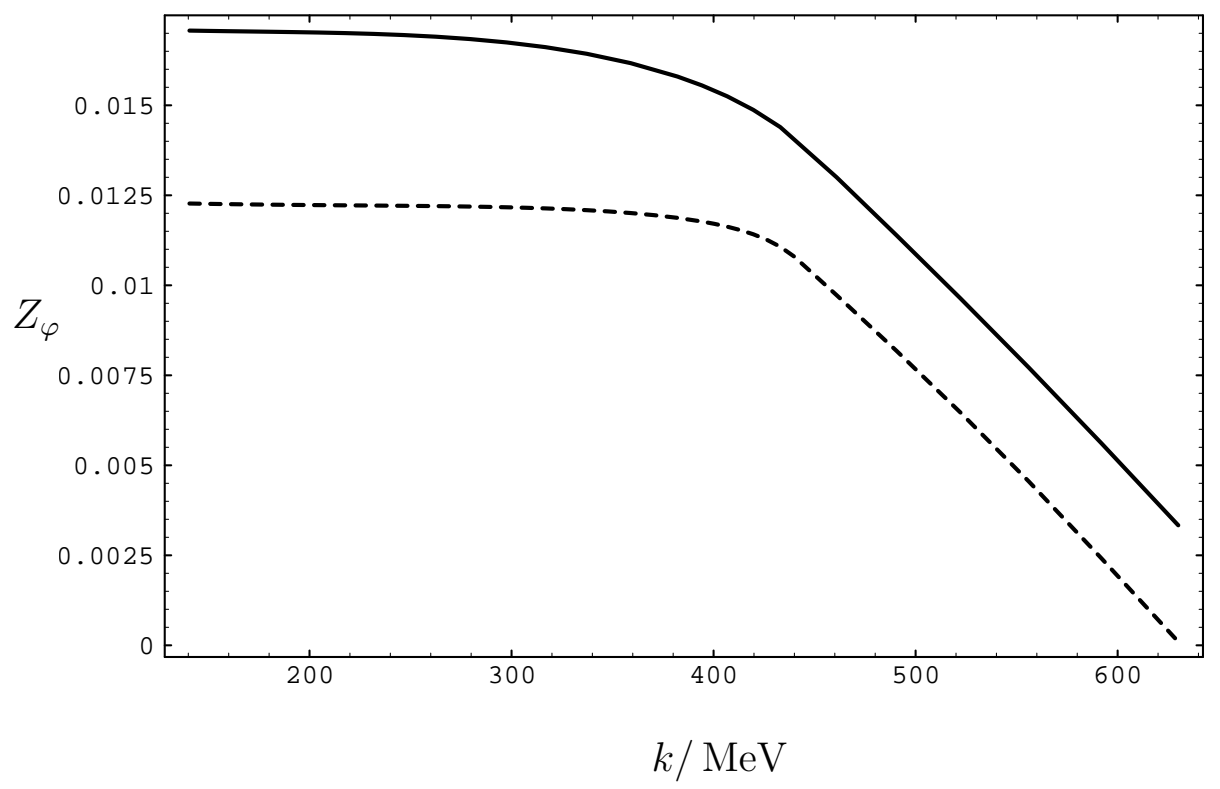

Figure 3: Evolution of $Z_{\varphi}$ with $k$, for the $U_{L}(2) \times U_{R}(2)$ model with $k_{\varphi}=630 \mathrm{MeV}, \tilde{\epsilon}_{0}=0.01$, $\lambda_{1}\left(k_{\varphi}\right)=\lambda_{2}\left(k_{\varphi}\right)=0$ and two values $h^{2}\left(k_{\varphi}\right)=300$ (solid line) and $h^{2}\left(k_{\varphi}\right)=10^{4}$ (dashed line).

$k_{\varphi}=630 \mathrm{MeV}, \tilde{\epsilon}_{0}=0.01, \lambda_{1}\left(k_{\varphi}\right)=\lambda_{2}\left(k_{\varphi}\right)=0$ and two values of $h^{2}\left(k_{\varphi}\right)=300$ and $10^{4}$. We note that $Z_{\varphi}$ increases strongly in the symmetric regime for $k>450 \mathrm{MeV}$ and stabilizes for low values of $k$. Again, the final value at $k=k_{f}$ does not depend very much on the initial conditions for $h^{2}$.

\section{Evolution equation for the Yukawa coupling and fermion anomalous dimension}

To determine the evolution equation for the Yukawa coupling and the fermionic wave function renormalization constant we will turn to a field configuration $\left(\varphi=\varphi^{*}\right)$

$$
\begin{aligned}
\varphi_{a b}(x) & =\varphi \delta_{a b} \\
\psi_{a}^{\hat{\alpha}}(x) & =\psi_{a}^{\hat{\alpha}} e^{-i Q x} \\
\bar{\psi}_{\hat{\alpha}}^{a}(x) & =\bar{\psi}_{\hat{\alpha}}^{a} e^{i Q x} .
\end{aligned}
$$

Furthermore, we will approximate the momentum dependence of the Yukawa coupling by $\bar{h}_{k}\left(-q, q^{\prime}\right) \simeq \bar{h}_{k}\left(\frac{q+q^{\prime}}{2}\right)$. This amounts to neglecting its dependence on the external scalar momentum $\frac{q-q^{\prime}}{2}$ in the Yukawa vertex in (3.3). Accordingly, the renormalized Yukawa coupling is defined via

$$
h_{k}(q)=k^{\frac{d}{2}-2} Z_{\psi, k}^{-1}(q) Z_{\varphi, k}^{-\frac{1}{2}}(0) \bar{h}_{k}(q)
$$


The matrix of second functional derivatives of $\Gamma_{k}$ simplifies considerably for the above configuration. Omitting spinor indices one finds

$$
\frac{\delta^{2} \Gamma_{k}}{\delta \bar{\psi}^{a}(q) \delta \psi_{b}\left(q^{\prime}\right)}=\left(Z_{\psi, k}(q) \phi+\bar{h}_{k}(q) \varphi \bar{\gamma}\right)(2 \pi)^{d} \delta\left(q-q^{\prime}\right) \delta_{a}^{b} .
$$

The derivation of the flow equations for $\bar{h}$ and $Z_{\psi}$ follows similar lines as for the scalar anomalous dimension discussed in section 4 . For details of the calculation we refer to appendix F. Neglecting the effects of the chiral anomaly $(\bar{\nu}=0)$ as well as the momentum dependence of the wave function renormalizations and the Yukawa coupling we find in the limit $Q \rightarrow 0$

$$
\begin{aligned}
\frac{\partial}{\partial t} h^{2} & =\left[d-4+2 \eta_{\psi}+\eta_{\varphi}\right] h^{2}-\frac{4}{N} v_{d} h^{4}\left\{N^{2} l_{1,1}^{(F B) d}\left(\frac{1}{N} \kappa h^{2}, \epsilon ; \eta_{\psi}, \eta_{\varphi}\right)\right. \\
& \left.-\left(N^{2}-1\right) l_{1,1}^{(F B) d}\left(\frac{1}{N} \kappa h^{2}, \epsilon+\kappa \lambda_{2} ; \eta_{\psi}, \eta_{\varphi}\right)-l_{1,1}^{(F B) d}\left(\frac{1}{N} \kappa h^{2}, \epsilon+2 \kappa \lambda_{1} ; \eta_{\psi}, \eta_{\varphi}\right)\right\}
\end{aligned}
$$

where

$$
\begin{array}{r}
l_{n_{1}, n_{2}}^{(F B) d}\left(w_{1}, w_{2} ; \eta_{\psi}, \eta_{\varphi}\right)=l_{n_{1}, n_{2}}^{(F B) d}\left(w_{1}, w_{2}\right)-\eta_{\psi} \breve{l}_{n_{1}, n_{2}}^{(F B) d}\left(w_{1}, w_{2}\right)-\eta_{\varphi} \hat{l}_{n_{1}, n_{2}}^{(F B) d}\left(w_{1}, w_{2}\right) \\
=-\frac{1}{2} k^{2\left(n_{1}+n_{2}\right)-d} \int_{0}^{\infty} d x x^{\frac{d}{2}-1} \frac{\widehat{\partial}}{\partial t}\left\{\frac{1}{\left[P_{F}(x)+k^{2} w_{1}\right]^{n_{1}}\left[P(x)+k^{2} w_{2}\right]^{n_{2}}}\right\} .
\end{array}
$$

Similarly, the fermionic anomalous dimension reads

$$
\begin{aligned}
\eta_{\psi} & =\frac{4}{N} \frac{v_{d}}{d} h^{2}\left\{N^{2} m_{1,2}^{(F B) d}\left(\frac{1}{N} h^{2} \kappa, \epsilon ; \eta_{\psi}, \eta_{\varphi}\right)+m_{1,2}^{(F B) d}\left(\frac{1}{N} h^{2} \kappa, \epsilon+2 \kappa \lambda_{1} ; \eta_{\psi}, \eta_{\varphi}\right)\right. \\
& \left.+\left(N^{2}-1\right) m_{1,2}^{(F B) d}\left(\frac{1}{N} h^{2} \kappa, \epsilon+\kappa \lambda_{2} ; \eta_{\psi}, \eta_{\varphi}\right)\right\}
\end{aligned}
$$

with

$$
\begin{array}{r}
m_{n_{1}, n_{2}}^{(F B) d}\left(w_{1}, w_{2} ; \eta_{\psi}, \eta_{\varphi}\right)=m_{n_{1}, n_{2}}^{(F B) d}\left(w_{1}, w_{2}\right)-\eta_{\psi} \check{m}_{n_{1}, n_{2}}^{(F B) d}\left(w_{1}, w_{2}\right)-\eta_{\varphi} \hat{m}_{n_{1}, n_{2}}^{(F B) d}\left(w_{1}, w_{2}\right) \\
=-\frac{1}{2} k^{2\left(n_{1}+n_{2}-1\right)-d} \int_{0}^{\infty} d x x^{\frac{d}{2}} \frac{\widehat{\partial}}{\partial t}\left\{\frac{1+r_{F}(x)}{\left[P_{F}(x)+k^{2} w_{1}\right]^{n_{1}}} \frac{\dot{P}(x)}{\left[P(x)+k^{2} w_{2}\right]^{n_{2}}}\right\}
\end{array}
$$

In summary, the equations (4.9) and (5.6) constitute a linear system for $\eta_{\varphi}$ and $\eta_{\psi}$ with solution

$$
\begin{aligned}
\eta_{\varphi} & =\frac{A_{1}\left(1+A_{6}\right)-A_{3} A_{4}}{\left(1+A_{2}\right)\left(1+A_{6}\right)-A_{3} A_{5}} \\
\eta_{\psi} & =\frac{A_{4}\left(1+A_{2}\right)-A_{1} A_{5}}{\left(1+A_{2}\right)\left(1+A_{6}\right)-A_{3} A_{5}}
\end{aligned}
$$

where $A_{1}, \ldots, A_{6}$ are defined by writing (4.9) and (5.6) in an obvious notation as

$$
\begin{aligned}
& \eta_{\varphi}=A_{1}-A_{2} \eta_{\varphi}-A_{3} \eta_{\psi} \\
& \eta_{\psi}=A_{4}-A_{5} \eta_{\varphi}-A_{6} \eta_{\psi} .
\end{aligned}
$$




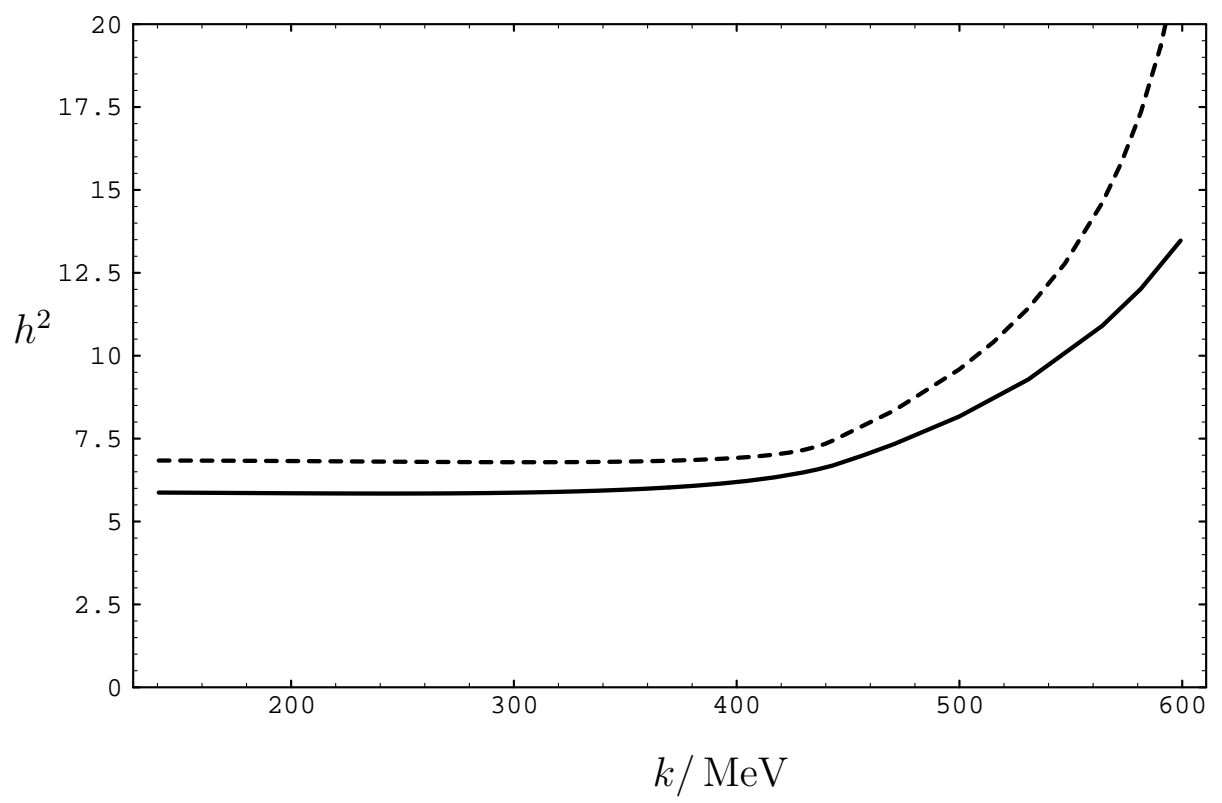

Figure 4: Dependence of $h^{2}$ on $k$, for the $U_{L}(2) \times U_{R}(2)$ model with two different initial values, $h^{2}\left(k_{\varphi}\right)=300$ (solid line) and $h^{2}\left(k_{\varphi}\right)=10^{4}$ (dashed line). We use $k_{\varphi}=630 \mathrm{MeV}, \tilde{\epsilon}_{0}=0.01$ and $\lambda_{1}\left(k_{\varphi}\right)=\lambda_{2}\left(k_{\varphi}\right)=0$.

We note that in four dimensions the integrals

$$
l_{1,1}^{(F B) 4}(0,0)=m_{4}^{(F) 4}(0)=m_{1,2}^{(F B) 4}(0,0)=1
$$

are independent of the particular choice of the infrared cutoff. We therefore find in the limit of small masses $\kappa, \epsilon$ in both regimes to leading order in the coupling constants the known $[16,17]$ perturbative one-loop results for both anomalous dimensions:

$$
\begin{aligned}
& \eta_{\varphi}=\frac{N_{c}}{8 \pi^{2}} h^{2} \\
& \eta_{\psi}=\frac{N}{16 \pi^{2}} h^{2} .
\end{aligned}
$$

This in turn yields the correct perturbative one-loop result

$$
\frac{\partial}{\partial t} h^{2}=\left(2 \eta_{\psi}+\eta_{\varphi}\right) h^{2}=\frac{N+N_{c}}{8 \pi^{2}} h^{4}
$$

We observe that for large $h^{2}$ the running of the Yukawa coupling is very fast due to the term $\sim h^{4}$ in the flow equation (5.4). This explains why different large values of $h^{2}\left(k_{\varphi}\right)$ lead to very similar results for $h_{R}^{2}=h^{2}(0)$. We demonstrate this in fig. 4 where two different initial values $h^{2}\left(k_{\varphi}\right)=300$ and $h^{2}\left(k_{\varphi}\right)=10^{4}$ are compared. Fig. 5 shows the corresponding evolution of $Z_{\psi}(k)$. 


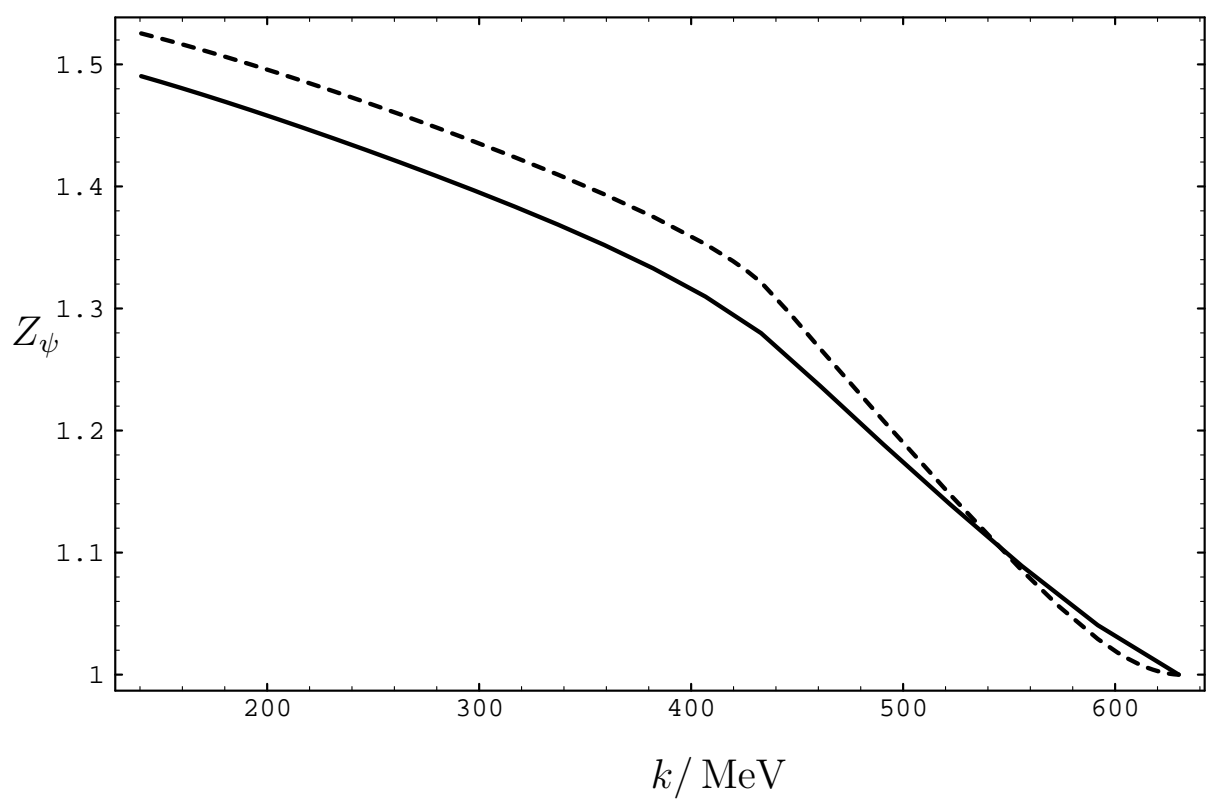

Figure 5: Running of $Z_{\psi}$ with $k$, for the $U_{L}(2) \times U_{R}(2)$ model with two different initial values $h^{2}\left(k_{\varphi}\right)=$ 300 (solid line), $h^{2}\left(k_{\varphi}\right)=10^{4}$ (dashed line), and $k_{\varphi}=630 \mathrm{MeV}, \tilde{\epsilon}_{0}=0.01, \lambda_{1}\left(k_{\varphi}\right)=\lambda_{2}\left(k_{\varphi}\right)=0$.

\section{The chiral anomaly}

So far we have considered the somewhat unrealistic limit $\bar{\nu} \rightarrow 0$ where the effects of the chiral anomaly are neglected. In view of the large value of $\nu_{R}(2.22)$ as compared to $k_{\varphi} \simeq 630 \mathrm{MeV}$, however, it appears that the opposite limit, $\bar{\nu} \rightarrow \infty$, should be closer to reality. For $N=2$ it is straightforward to take the effects of the chiral anomaly in this limit into account. To see this we notice that the complex $(\mathbf{2}, \mathbf{2})$ representation $\varphi$ of the global symmetry group $S U_{L}(2) \times S U_{R}(2) \simeq O(4)$ decomposes into two irreducible real vector representations of $O(4)$ (cf. the discussion at the end of appendix B):

$$
\varphi=\frac{1}{2}\left(\sigma-i \eta^{\prime}\right)+\frac{1}{2}\left(a^{k}+i \pi^{k}\right) \tau_{k} .
$$

By taking $\bar{\nu} \rightarrow \infty$ while keeping $\bar{m}^{2}-\frac{1}{2} \bar{\nu}$ (or $\bar{\mu}^{2}+\frac{1}{2} \bar{\nu}$ ) fixed, $m_{a}$ and $m_{\eta^{\prime}}$ diverge and the four corresponding mesons decouple. Hence, we are left with the real vector representation $\vec{\phi}=\left(\sigma, \pi_{1}, \pi_{2}, \pi_{3}\right)$ of $O(4)$. Its potential reads in the symmetric regime

$$
U_{k}=\frac{1}{2}\left(\bar{m}^{2}-\frac{1}{2} \bar{\nu}\right) \phi_{a} \phi^{a}+\frac{1}{8} \lambda_{1}\left(\phi_{a} \phi^{a}\right)^{2}
$$

and similarly in the SSB regime. We therefore end up with the $O(4)$ symmetric linear sigma model coupled to fermions. The flow equations in the symmetric regime are given 
by $[10,23]$

$$
\begin{aligned}
\frac{\partial \epsilon}{\partial t} & =-\left(2-\eta_{\varphi}\right) \epsilon-2 v_{d}\left\{6 \lambda_{1} l_{1}^{d}\left(\epsilon ; \eta_{\varphi}\right)-2^{\frac{d}{2}} N_{c} h^{2} l_{1}^{(F) d}\left(\eta_{\psi}\right)\right\} \\
\frac{\partial \lambda_{1}}{\partial t} & =\left(d-4+2 \eta_{\varphi}\right) \lambda_{1}+2 v_{d}\left\{12 \lambda_{1}^{2} l_{2}^{d}\left(\epsilon ; \eta_{\varphi}\right)-2^{\frac{d}{2}-1} N_{c} h^{4} l_{2}^{(F) d}\left(\eta_{\psi}\right)\right\} \\
\frac{\partial h^{2}}{\partial t} & =\left(d-4+2 \eta_{\psi}+\eta_{\varphi}\right) h^{2}-4 v_{d} h^{4} l_{1,1}^{(F B) d}\left(0, \epsilon ; \eta_{\psi}, \eta_{\varphi}\right) \\
\eta_{\varphi} & =2^{\frac{d}{2}+2} \frac{v_{d}}{d} N_{c} h^{2} m_{4}^{(F) d}\left(0 ; \eta_{\psi}\right) \\
\eta_{\psi} & =8 \frac{v_{d}}{d} h^{2} m_{1,2}^{(F B) d}\left(0, \epsilon ; \eta_{\psi}, \eta_{\varphi}\right)
\end{aligned}
$$

where $\epsilon$ is defined here by $\epsilon=Z_{\varphi}^{-1} k^{-2}\left(\bar{m}^{2}-\frac{1}{2} \bar{\nu}\right)$. For the SSB regime we find

$$
\begin{aligned}
\frac{\partial \kappa}{\partial t} & =\left(2-d-\eta_{\varphi}\right) \kappa+2 v_{d}\left\{3 l_{1}^{d}\left(\eta_{\varphi}\right)+3 l_{1}^{d}\left(2 \lambda_{1} \kappa ; \eta_{\varphi}\right)-2^{\frac{d}{2}} N_{c} \frac{h^{2}}{\lambda_{1}} l_{1}^{(F) d}\left(\frac{1}{2} h^{2} \kappa ; \eta_{\psi}\right)\right\} \\
\frac{\partial \lambda_{1}}{\partial t} & =\left(d-4+2 \eta_{\varphi}\right) \lambda_{1} \\
& +2 v_{d}\left\{3 \lambda_{1}^{2} l_{2}^{d}\left(\eta_{\varphi}\right)+9 \lambda_{1}^{2} l_{2}^{d}\left(2 \lambda_{1} \kappa ; \eta_{\varphi}\right)-2^{\frac{d}{2}-1} N_{c} h^{4} l_{2}^{(F) d}\left(\frac{1}{2} h^{2} \kappa ; \eta_{\psi}\right)\right\} \\
\frac{\partial h^{2}}{\partial t} & =\left(d-4+2 \eta_{\psi}+\eta_{\varphi}\right) h^{2} \\
& -2 v_{d} h^{4}\left\{3 l_{1,1}^{(F B) d}\left(\frac{1}{2} h^{2} \kappa, 0 ; \eta_{\psi}, \eta_{\varphi}\right)-l_{1,1}^{(F B) d}\left(\frac{1}{2} h^{2} \kappa, 2 \lambda_{1} \kappa ; \eta_{\psi}, \eta_{\varphi}\right)\right\} \\
\eta_{\varphi} & =4 \frac{v_{d}}{d}\left\{4 \kappa \lambda_{1}^{2} m_{2,2}^{d}\left(0,2 \lambda_{1} \kappa ; \eta_{\varphi}\right)+2^{\frac{d}{2}} N_{c} h^{2} m_{4}^{(F) d}\left(\frac{1}{2} h^{2} \kappa ; \eta_{\psi}\right)\right\} \\
\eta_{\psi} & =2 \frac{v_{d}}{d} h^{2}\left\{3 m_{1,2}^{(F B) d}\left(\frac{1}{2} h^{2} \kappa, 0 ; \eta_{\psi}, \eta_{\varphi}\right)+m_{1,2}^{(F B) d}\left(\frac{1}{2} h^{2} \kappa, 2 \lambda_{1} \kappa ; \eta_{\psi}, \eta_{\varphi}\right)\right\}
\end{aligned}
$$

The difference between the results of the $O(4)$ model $(\bar{\nu} \rightarrow \infty)$ and the $U_{L}(2) \times U_{R}(2)$ model $(\bar{\nu}=0)$ can be taken as a measure for the uncertainty due to the rough treatment of the anomaly in the present work. Here the $U_{L}(2) \times U_{R}(2)$ model exhibits the effects of additional scalar degrees of freedom beyond the pions (and the $\sigma$-mode). Since the additional modes are relatively heavy, the $O(4)$ model should be closer to reality. The best model with $N=3$, where the strange quark mass and the chiral anomaly are properly taken into account, is expected to deviate from the $O(4)$ model in the same direction as the $U_{L}(2) \times U_{R}(2)$ model. Since there are three light quark flavors in nature with masses smaller than $k_{\varphi}$ one might naively expect the case $N=3$ to correspond to the most realistic description of the real world. However, we have neglected quark masses and in particular the strange quark mass in this work. In the $S U_{L}(3) \times S U_{R}(3) \times U_{V}(1)$ model the four $K$-mesons will therefore appear as massless Goldstone degrees of freedom which will unnaturally drive the evolution of all parameters even at scales much lower than their physical masses of approximately $500 \mathrm{MeV}$. The same holds, of course, for the three 


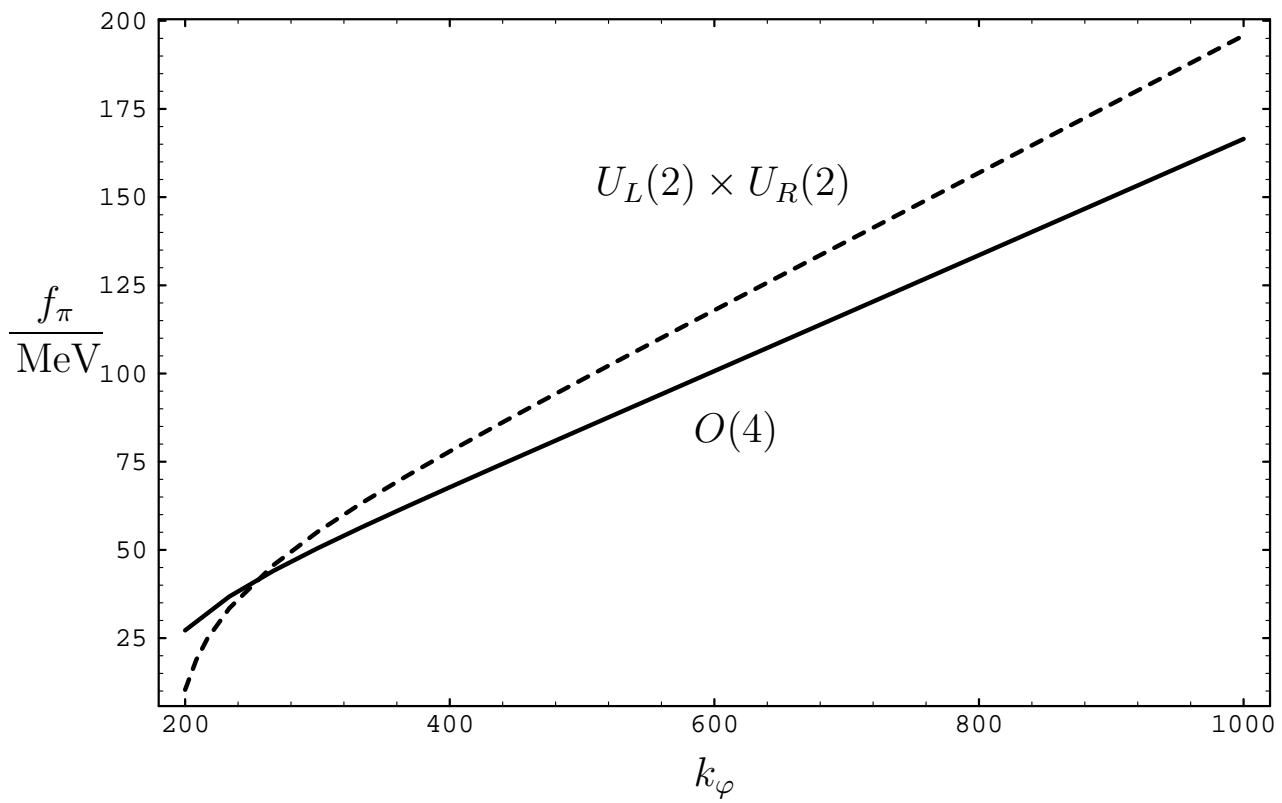

Figure 6: The pion decay constant $f_{\pi}$ as a function of $k_{\varphi}$ for $\lambda_{1}\left(k_{\varphi}\right)=\lambda_{2}\left(k_{\varphi}\right)=0, h^{2}\left(k_{\varphi}\right)=300$ and $\tilde{\epsilon}_{0}=0.01$.

pions. The effects of their physical masses $m_{\pi} \simeq 140 \mathrm{MeV}$ can, however, be mimicked by stopping the running for $k_{f}=m_{\pi}$. We therefore expect the case $N=2$ to yield more realistic results than $N=3$ as long as the strange quark mass is neglected. In addition, we note that for $N=3$ the scalar self coupling $\lambda_{1}$ turns negative for positive but small values of $\bar{m}^{2}$. This happens despite the fact that $\lambda_{1}$ has acquired first a large positive value due to the strong initial Yukawa coupling. The cause is a large value of $\lambda_{2}$ which can drive $\lambda_{1}$ negative when the scalar loop contributions to the running of $\lambda_{1}$ become numerically important around the scale $k_{s}$. We interpret this quartic instability of the truncation (2.7) of the effective potential as a signal for a first order phase transition in the mass parameter even within the $U_{L}(3) \times U_{R}(3)$ model without chiral anomaly. A proper treatment of the case $N=3$ therefore requires a more general truncation of the effective potential [24]. We will leave this problem for future work. However, we would like to point out that the inclusion of the cubic (for $N=3$ ) $\xi$-term into the potential will change the phase transition to first order anyway. One expects that the vacuum expectation value of $\varphi$ jumps discontinuously to a finite value already for a large value of $\epsilon$ such that the scalar contributions to the evolution of $\lambda_{1}$ never become strong enough to turn it negative.

The difference between the $O(4)$ model and the $U_{L}(2) \times U_{R}(2)$ model is exemplified in fig. 6. There we show the "prediction" of $f_{\pi}$ as a function of the initial scale $k_{\varphi}$. The difference between the two models is not very large. We observe for not too small values of $k_{\varphi}$ a linear behavior $f_{\pi} \sim k_{\varphi}$. For fixed initial values of the dimensionless parameters 
like $\tilde{\epsilon}=\bar{m}^{2} Z_{\psi}^{2} / k_{\varphi}^{2}, h^{2}$ etc., this proportionality follows on pure dimensional grounds if no other mass scale is present. The bending of the curves for small $k_{\varphi}$ is therefore purely a consequence of the additional infrared cutoff $k_{f}=m_{\pi}$ which obviously plays a negligible role for a realistic size of $k_{\varphi}$. Looking at fig. 6 the reader may prematurely conclude that the predictive power for $f_{\pi}$ is severely limited by the arbitrariness of the choice of the scale $k_{\varphi}$ from where on the meson description is used. We should emphasize that for a full treatment along the lines of $[14,15]$ this is actually not the case: If one lowers the transition scale $k_{\varphi}$ more fluctuations are included in the momentum range $q^{2}>k_{\varphi}^{2}$ where a quark-gluon picture is used. As a result, the pole like structure in the effective four-quark interaction becomes stronger and $\bar{m}^{2}$ therefore decreases. This is the same effect as found in the quark-meson picture used for the fluctuations with $q^{2}<k_{\varphi}^{2}$ : the mass term $\bar{m}^{2}$ decreases with smaller $k$ as a result of the Yukawa coupling to the quarks. In the limit where the pole like structure dominates the evolution of $\tilde{G}(0)=\frac{1}{2 \bar{m}^{2}}$ in the quark gluon picture the running of $\bar{m}^{2}$ is identical in both pictures for $k$ larger or smaller than $k_{\varphi}$. The initial value $\bar{m}^{2}\left(k_{\varphi}\right)$ as a function of $k_{\varphi}$ follows therefore the same renormalization group trajectory as given by the flow equation (3.14). In this ideal case the choice of the transition scale $k_{\varphi}$ does not affect the results, since the initial conditions move on trajectories of constant physics. In practice, this ideal scenario will often not be fully realized, since different types of fluctuations are included in the quark-gluon and the quark-meson description. The dependence of the results on $k_{\varphi}$ can then be used as a quantitative check of the reliability of the employed truncations for the effective action.

\section{Infrared stability and predictive power for $f_{\pi}$}

Comparing the results for $f_{\pi}$ from fig. 6 with the experimental value $f_{\pi}=93 \mathrm{MeV}$ we find a surprisingly good agreement for $k_{\varphi}=630 \mathrm{MeV}$ as infered from ref. [14]. The question arises to what extent this result depends on the particular choice of initial values at the scale $k_{\varphi}$. In principle, the values of the parameters of the quark-meson system at the scale $k_{\varphi}$ can be computed from QCD $[14,15]$. In practice, however, many quantities will not be available with high accuracy, since one has to deal with a problem involving strong interactions. If $f_{\pi}$ would depend very sensitively on such quantities, a computation of $f_{\pi}$ with satisfactory precision would be extremely difficult. We will argue in this section that for small enough $Z_{\varphi}$ the opposite situation is realized. In this event the prediction for $f_{\pi}$ turns out to be almost independent of the initial values of many couplings. The reason is that a small $Z_{\varphi}$ corresponds to a strong Yukawa interaction. The large value of $h$ induces then a very fast running of almost all couplings towards values which are determined by an infrared attractive behavior. More precisely, the ratios $\lambda_{1} / h^{2}$ and $\lambda_{2} / h^{2}$ are determined by infrared fixed points of the type first found in the electroweak standard model [25]. This explains the insensitivity with respect to the initial values as demonstrated in fig. 2. The Yukawa coupling itself is also strongly renormalized and predicted to be in the vicinity 
of the upper bound of the relevant infrared interval ${ }^{5}[26]$. Here the upper bound of the infrared interval is essentially determined by the scale $k_{s}$ where spontaneous symmetry breaking sets in and the effective quark masses constitute an infrared cutoff. This can clearly be observed in fig. 4 where also the insensitivity with respect to the initial value $h\left(k_{\varphi}\right)$ becomes apparent. The only relevant parameter will turn out to be the ratio

$$
\tilde{\epsilon}_{0}=\frac{\epsilon\left(k_{\varphi}\right)}{h^{2}\left(k_{\varphi}\right)}=\frac{\bar{m}^{2}\left(k_{\varphi}\right) Z_{\psi}^{2}\left(k_{\varphi}\right)}{k_{\varphi}^{2}} .
$$

This value determines $k_{s}$ and $f_{\pi}$ as well as all other couplings at the scale $k_{f}$.

For small enough $Z_{\varphi}$ one starts in a regime where $\epsilon=m^{2} / k^{2}=\bar{m}^{2} Z_{\varphi}^{-1} k^{-2}$ is large. For large $\epsilon$ the scalar fluctuations are suppressed by inverse powers of $\epsilon$ appearing in the threshold functions. Then the scalar fluctuations can be neglected and only quark fluctuations drive the flow of the couplings. This is the approximation used in ref. [14] which remains valid as long as $\epsilon \gg 1$. The Yukawa coupling $\bar{h}$ is normalized according to (3.7) as $\bar{h}(0) \equiv \bar{h}_{k_{\varphi}}(0,0)=1$. Consequently, the initial value for the renormalized Yukawa coupling is

$$
h_{0}^{2} \equiv h^{2}\left(k_{\varphi}\right)=\frac{1}{Z_{\varphi}\left(k_{\varphi}\right) Z_{\psi}^{2}\left(k_{\varphi}\right)} .
$$

We use here a normalization of the fermion kinetic term such that $Z_{\psi}\left(k_{\varphi}\right)=1$. For small $Z_{\varphi}\left(k_{\varphi}\right)$ we therefore start with a strong Yukawa coupling. In the limit $\epsilon \gg 1$ the flow equations simplify considerably. If we define $\tilde{\epsilon} \equiv \epsilon / h^{2}$ and $\tilde{\lambda}_{i} \equiv \lambda_{i} / h^{2}$ we find

$$
\begin{aligned}
\frac{\partial \tilde{\epsilon}}{\partial t} & =-(d-2) \tilde{\epsilon}+2^{\frac{d}{2}+1} v_{d} N_{c} \\
\frac{\partial \tilde{\lambda}_{1}}{\partial t} & =2^{\frac{d}{2}+1} v_{d} N_{c} h^{2}\left[\frac{2}{d} \tilde{\lambda}_{1}-\frac{1}{N}\right] \\
\frac{\partial \tilde{\lambda}_{2}}{\partial t} & =2^{\frac{d}{2}+2} v_{d} N_{c} h^{2}\left[\frac{1}{d} \tilde{\lambda}_{2}-\frac{1}{N}\right] \\
\frac{\partial h^{2}}{\partial t} & =(d-4) h^{2}+2^{\frac{d}{2}+2} \frac{v_{d}}{d} N_{c} h^{4} .
\end{aligned}
$$

In the following we will specialize to the case $d=4 .^{6}$

As a first observation we notice that the $\tilde{\lambda}_{1}-\tilde{\lambda}_{2}$ system exhibits an infrared fixed point given by

$$
\tilde{\lambda}_{1 *}=\frac{1}{2} \tilde{\lambda}_{2 *}=\frac{2}{N} .
$$

This fixed point corresponds exactly to the large $-N_{c}$ estimate of [17]. The explicit solution

\footnotetext{
${ }^{5}$ The infrared fixed point for $h$ is $h_{*}=0$ if no infrared cutoff is present. Due to a finite amount of running from $k_{\varphi}$ to $k_{s}$ this translates into an infrared interval.

${ }^{6}$ The system (7.3) remains solvable for general $d$.
} 
of the differential equations (7.3) reads

$$
\begin{aligned}
\tilde{\epsilon}(t) & =4 v_{4} N_{c}\left[1-e^{-2 t}\right]+\tilde{\epsilon}_{0} e^{-2 t} \\
\tilde{\lambda}_{1}(t) & =\frac{\frac{\lambda_{10}}{h_{0}^{2}}-8 \frac{N_{c}}{N} v_{4} h_{0}^{2} t}{1-4 N_{c} v_{4} h_{0}^{2} t} \\
\tilde{\lambda}_{2}(t) & =\frac{\frac{\lambda_{20}}{h_{0}^{2}}-16 \frac{N_{c}}{N} v_{4} h_{0}^{2} t}{1-4 N_{c} v_{4} h_{0}^{2} t} \\
h^{2}(t) & =\frac{h_{0}^{2}}{1-4 N_{c} v_{4} h_{0}^{2} t}
\end{aligned}
$$

with $\tilde{\epsilon}_{0} \equiv \tilde{\epsilon}(t=0)$, etc. The system crosses into the SSB regime when $\tilde{\epsilon}\left(t_{s}\right)=0$, corresponding to a scale

$$
t_{s}=\frac{1}{2} \ln \left[1-\frac{\tilde{\epsilon}_{0}}{4 N_{c} v_{4}}\right], \quad k_{s}^{2}=\left(1-\frac{8 \pi^{2}}{3} \tilde{\epsilon}_{0}\right) k_{\varphi}^{2} .
$$

Here we have used $v_{4}=\left(32 \pi^{2}\right)^{-1}$ and $N_{c}=3$ in the last expression. Around the scale $k_{s}$ our approximation (large $\epsilon$ ) breaks down. Nevertheless, it becomes apparent already at this stage that values of $\tilde{\epsilon}_{0}$ substantially larger than 0.04 are incompatible with chiral symmetry breaking, since $\tilde{\epsilon}$ would remain positive for all $k$ in this case. For large $\tilde{\epsilon}_{0}$ the effect of the quark fluctuations is simply not strong enough to turn the scalar mass term negative. We notice that $\tilde{\epsilon}_{0} \ll 1$ for $\bar{m}\left(k_{\varphi}\right) \sim \mathcal{O}(100 \mathrm{MeV})$ and $Z_{\psi}\left(k_{\varphi}\right) \lesssim 1$. If we furthermore assume $-4 N_{c} v_{4} h_{0}^{2} t_{s} \gg 1$ or

$$
\tilde{\epsilon}_{0} \gg 4 N_{c} v_{4}\left[1-\exp \left\{-\frac{1}{2 N_{c} v_{4} h_{0}^{2}}\right\}\right],
$$

we find for not too large $\lambda_{10} / h_{0}^{2}, \lambda_{20} / h_{0}^{2}$

$$
\tilde{\lambda}_{1}\left(t_{s}\right) \simeq \tilde{\lambda}_{1 *}, \quad \tilde{\lambda}_{2}\left(t_{s}\right) \simeq \tilde{\lambda}_{2 *}, \quad h^{2}\left(t_{s}\right) \simeq\left[2 N_{c} v_{4} \ln \frac{4 N_{c} v_{4}}{4 N_{c} v_{4}-\tilde{\epsilon}_{0}}\right]^{-1}
$$

This result may be interpreted as follows: Even though (7.6) should only give an approximate estimate for the entering point into the SSB regime this is sufficient to imply that $\tilde{\lambda}_{1}$ and $\tilde{\lambda}_{2}$ approximately reach their fixed point long before $\epsilon$ goes through zero provided (7.7) is fulfilled. Furthermore $h^{2}$ becomes asymptotically independent of $h_{0}^{2}$ and is only a function of $\tilde{\epsilon}_{0}$. We therefore conclude that for small $Z_{\varphi}\left(k_{\varphi}\right)$ the system is governed by an infrared fixed point of $\tilde{\lambda}_{1}$ and $\tilde{\lambda}_{2}$ in the symmetric regime and looses almost all its information on the initial values at $k=k_{\varphi}$. Infrared quantities of the SSB regime like $f_{\pi}$ or meson masses will therefore merely depend on $\tilde{\epsilon}_{0}$ for a given $k_{\varphi}$. It is straightforward to see that the same analysis holds for the $O(4)$ model discussed in section 6 . The approximate flow equations for $\tilde{\epsilon}, \tilde{\lambda}_{1}$ and $h^{2}$ are the same as (7.3). The difference between the $U_{L}(2) \times U_{R}(2)$ and the $O(4)$ model arises principally from the behavior of the running couplings around the scale $k_{s}$. 


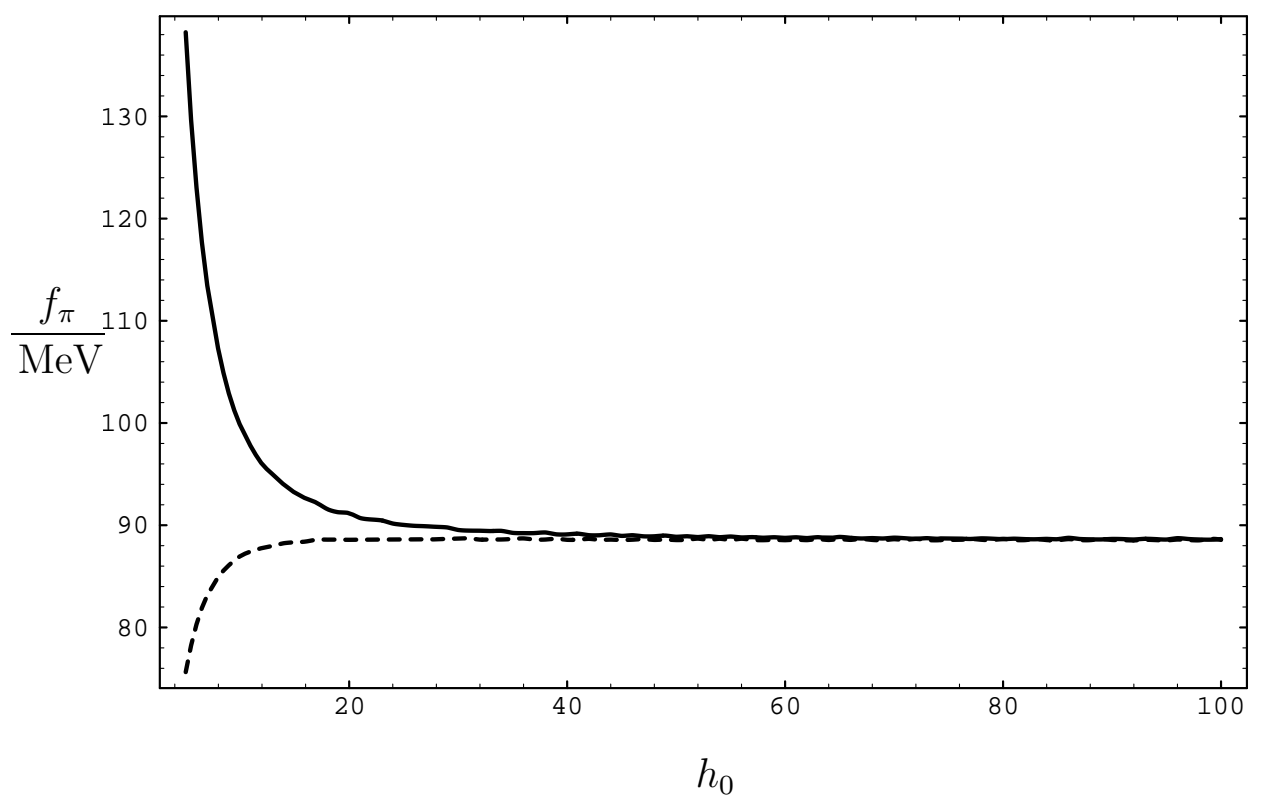

Figure 7: The $O(4)$ model pion decay constant $f_{\pi}$ as a function of $h_{0} \equiv h\left(k_{\varphi}\right)$ for $k_{\varphi}=630 \mathrm{MeV}$, $\tilde{\epsilon}_{0}=0.02$ and $\lambda_{1}\left(k_{\varphi}\right)=0$ (solid line) as well as $\lambda_{1}\left(k_{\varphi}\right)=h_{0}^{2}$ (dashed line).

For larger values of $Z_{\varphi}\left(k_{\varphi}\right)$ or smaller values of $h\left(k_{\varphi}\right)$ the attraction of the infrared fixed points becomes weaker. As a consequence, the dependence of $f_{\pi}$ on the initial values of the couplings becomes more important as demonstrated in fig. 7. We conclude that for $h\left(k_{\varphi}\right)$ substantially smaller than ten it will become more and more difficult to obtain an accurate prediction for $f_{\pi}$. On the other hand, fig. 7 clearly shows the approximate independence of $f_{\pi}$ on $h^{2}\left(k_{\varphi}\right)$ or $\lambda_{1}\left(k_{\varphi}\right)$ if $h^{2}\left(k_{\varphi}\right)$ exceeds 300 .

An additional aspect of strong Yukawa couplings concerns the error in $f_{\pi}$ due to the truncations of the quark-meson effective action. The effects of truncations in the scalar sector are diminished by the fact that scalar fluctuations are subdominant in the region of very large Yukawa couplings. A similar argument justifies the approximation of neglecting the terms proportional to the anomalous dimensions in the threshold functions. For the $m$-type functions this approximation is valid, since $A_{2}, A_{3}, A_{5}, A_{6} \ll 1$ in (5.8), (5.9). For $l$-type functions one might be worried that $\eta_{\varphi}$ and $\eta_{\psi}$ are large for the initial part of the running in the symmetric regime due to large values of $h^{2}$. However, because of the large values of $\epsilon$ in this range of scales the contributions from the $l$-type functions can be neglected altogether. For smaller values of $\epsilon$, i.e. for scales closer to $k_{s}$, the anomalous dimensions are expected to be already small (as indicated by figs. (3) and (4)). 


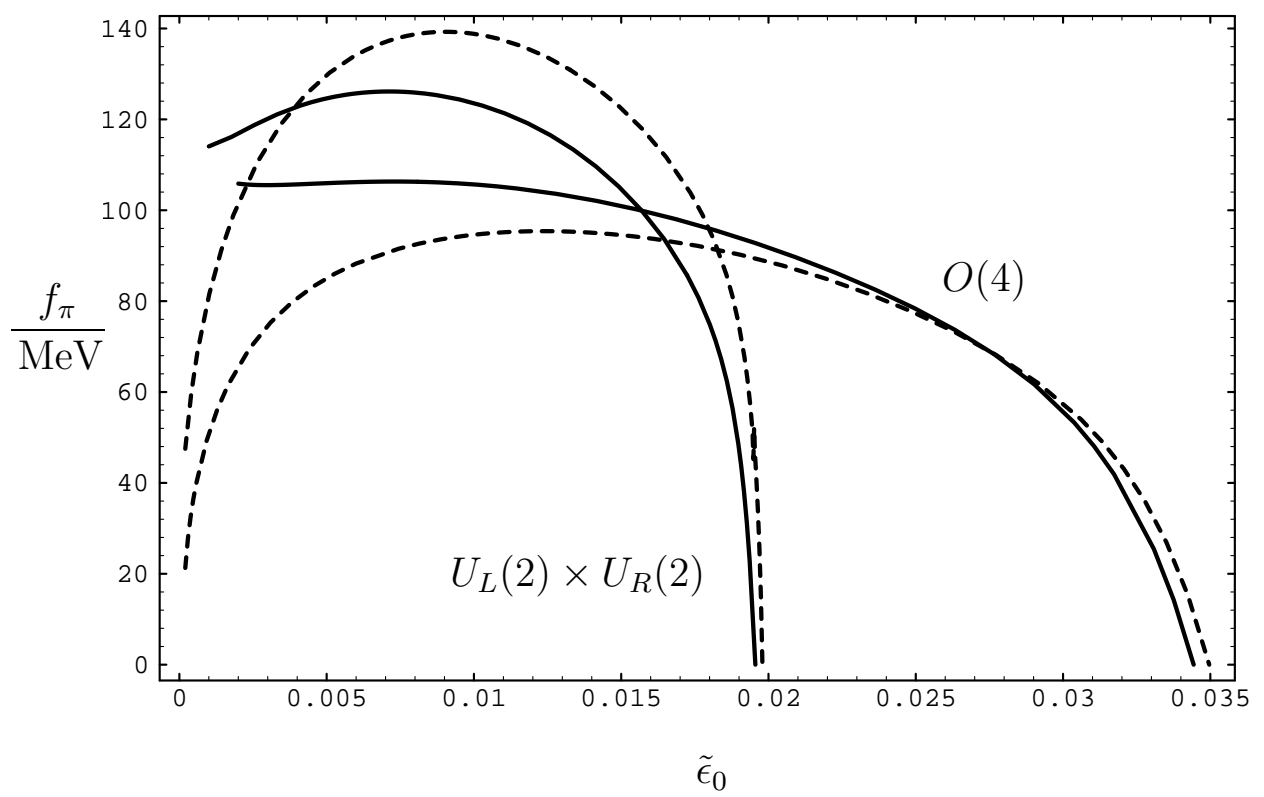

Figure 8: The pion decay constant $f_{\pi}$ as a function of $\tilde{\epsilon}_{0}$ for $k_{\varphi}=630 \mathrm{MeV}, \lambda_{1}\left(k_{\varphi}\right)=\lambda_{2}\left(k_{\varphi}\right)=0$ and $h^{2}\left(k_{\varphi}\right)=300$ (solid line) as well as $h^{2}\left(k_{\varphi}\right)=10^{4}$ (dashed line).

\section{Computation of $f_{\pi}$ for strong Yukawa coupling}

In the last section we have shown that for large enough Yukawa couplings, say $h^{2}\left(k_{\varphi}\right)>$ 200 , the value of $f_{\pi}$ only depends on the parameter $\tilde{\epsilon}\left(k_{\varphi}\right) \equiv \tilde{\epsilon}_{0}$ for given $k_{\varphi}$. We demonstrate this quantitatively in fig. 8 where we plot $f_{\pi}$ as a function of $\tilde{\epsilon}_{0}$, for both the $O(4)$ and the $U_{L}(2) \times U_{R}(2)$ model as well as for two different large initial values of $h^{2}$. The "prediction" for $f_{\pi}$ is rather insensitive to $h^{2}\left(k_{\varphi}\right)$ for moderate values of $\tilde{\epsilon}_{0} \cdot{ }^{7}$ For the $O(4)$ model we also observe an extended plateau where $f_{\pi}$ is not very sensitive to $\tilde{\epsilon}_{0}$ either. For this plateau the value of $f_{\pi}$ comes out between 80 and $100 \mathrm{MeV}$ which fits very well with the experimental value of $93 \mathrm{MeV}$. Also the renormalized Yukawa coupling $h_{R}=h\left(k_{f}\right)$, or, equivalently, the constituent quark mass $m_{q}=\frac{1}{2} h_{R} f_{\pi}$ depends essentially only on $\tilde{\epsilon}_{0}$. We show this in fig. 9, again for two different large values of $h^{2}\left(k_{\varphi}\right)$. Since both $h_{R}$ and $f_{\pi}$ are functions of only one parameter $\tilde{\epsilon}_{0}$, there arises a quantitative relation between those two quantities. Consider first the $O(4)$ model: For a constituent quark mass of $300 \mathrm{MeV}$ or $h_{R} \simeq 6.5$ we read off from fig. 9 that $\tilde{\epsilon}_{0} \simeq 0.02$. Inserting this into the plot of fig. 8 one obtains

$$
f_{\pi} \simeq 92 \mathrm{MeV} \text {. }
$$

\footnotetext{
${ }^{7}$ The decrease of $f_{\pi}$ for $h^{2}\left(k_{\varphi}\right)=10^{4}$ observed for unnaturally small values of $\tilde{\epsilon}_{0}$ results simply from the fact that the system enters almost immediately into the SSB regime, having no "time" for $Z_{\varphi}$ to grow much beyond $Z_{\varphi}\left(k_{\varphi}\right)$.
} 


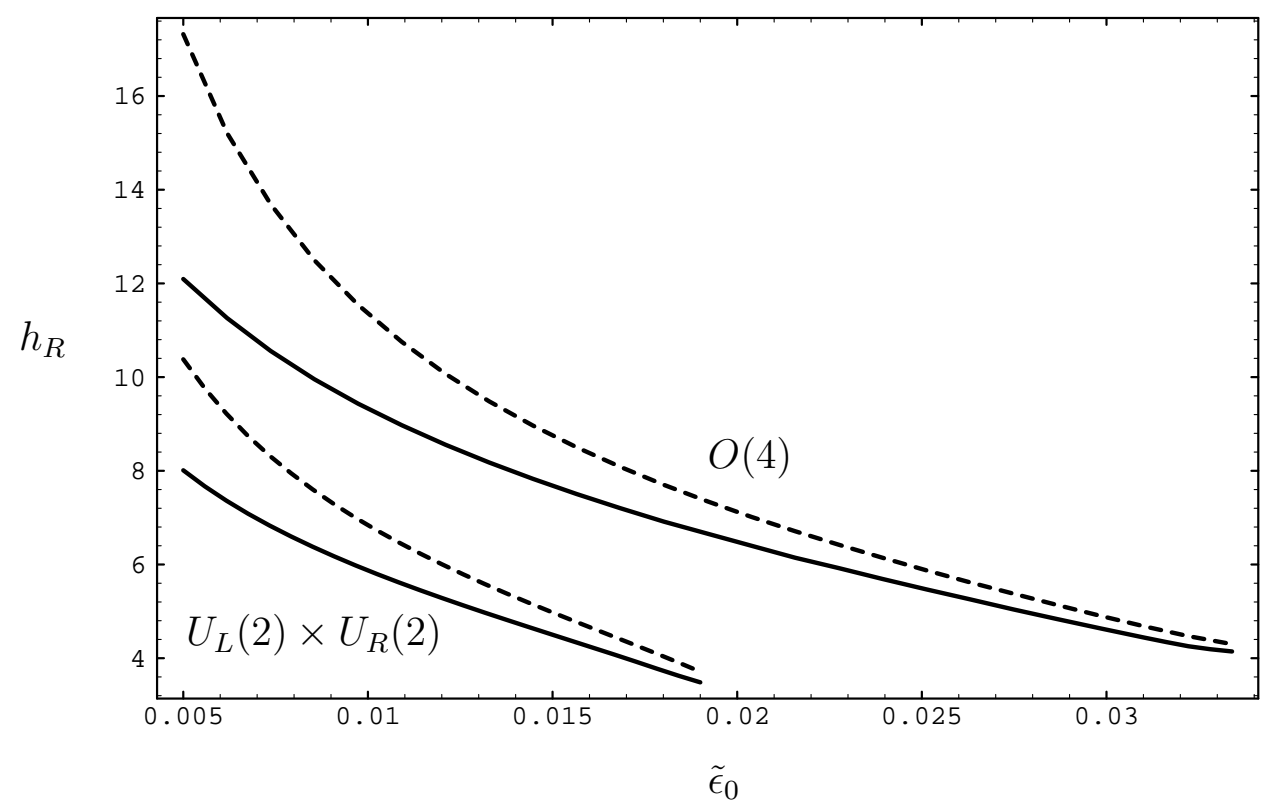

Figure 9: The renormalized Yukawa coupling $h_{R}$ as a function of $\tilde{\epsilon}_{0}$ for $k_{\varphi}=630 \mathrm{MeV}, \lambda_{1}\left(k_{\varphi}\right)=$ $\lambda_{2}\left(k_{\varphi}\right)=0$ and $h^{2}\left(k_{\varphi}\right)=300$ (solid lines), $h^{2}\left(k_{\varphi}\right)=10^{4}$ (dashed lines).

This value was obtained for $h^{2}\left(k_{\varphi}\right)=300$ and $\lambda_{1}\left(k_{\varphi}\right)=\lambda_{2}\left(k_{\varphi}\right)=0$, but it turns out to be not very different for $h^{2}\left(k_{\varphi}\right)=10^{4}$ or different initial values of $\lambda_{1}$ and $\lambda_{2}$. A similar procedure gives for the $U_{L}(2) \times U_{R}(2)$ model a value $\tilde{\epsilon}_{0} \simeq 0.008$ and in turn $f_{\pi} \simeq 126 \mathrm{MeV}$. Repeating this procedure for $k_{\varphi}=700 \mathrm{MeV}$ we can infer from table $1 \mathrm{a}$ guess of the uncertainty in $f_{\pi}$ that can be expected within the quark-meson model for large Yukawa couplings, as represented in the table by the values $h^{2}\left(k_{\varphi}\right)=300$ and $10^{4}$. On the other hand, a given value of $m_{q}$ also implies a minimal value $h_{\min }\left(k_{\varphi}\right)$ such that the evolution of $h(k)$ can reach the value $h_{R}=2 m_{q} / f_{\pi}$ at all. We assume here that the result for $\bar{m}^{2}\left(k_{\varphi}\right)$ of ref. [14] should not be off by more than a factor of four. We can therefore conclude that for $k_{\varphi}$ in the range $(630-700) \mathrm{MeV}$ there exists a lower bound on $\tilde{\epsilon}_{0}$, i.e. $\tilde{\epsilon}_{0} \gtrsim 0.01$. This in turn amounts for the $O(4)$ model to $h_{\min }\left(k_{\varphi}\right) \simeq 6.2,8.0,10.3$ for $m_{q}=250,300,350 \mathrm{MeV}$, respectively. The corresponding values in table 1 give an estimate for the maximal deviation of $f_{\pi}$ from its value for strong Yukawa coupling. For the $U_{L}(2) \times U_{R}(2)$ model a value $h^{2}\left(k_{\varphi}\right)=300$ is already near the lower limit of what is compatible with realistic values for $m_{q}$ and $\tilde{\epsilon}_{0}$ (cf. figure 9 ).

We can also invert these relations and look for the optimum value of $\tilde{\epsilon}_{0}$ and $h^{2}\left(k_{\varphi}\right)$ for fixed $f_{\pi}=93 \mathrm{MeV}$ and $m_{q}=300 \mathrm{MeV}$. One obtains for the $O(4)$ model and $k_{\varphi}=630 \mathrm{MeV}$

$$
\begin{aligned}
\tilde{\epsilon}_{0} & \simeq 0.02 \\
h^{2}\left(k_{\varphi}\right) & \simeq 280 .
\end{aligned}
$$

Within the simple QCD inspired model of ref. [14] the transition scale was found as 


\begin{tabular}{|c|c|c||c|c|c||c|c|c|}
\hline \multicolumn{2}{|c|}{} & \multicolumn{1}{c||}{$\frac{m_{q}}{\mathrm{MeV}}$} & \multicolumn{3}{c||}{300} & \multicolumn{3}{c|}{350} \\
\cline { 3 - 9 } \multicolumn{2}{c|}{} & $h^{2}\left(k_{\varphi}\right)$ & 64 & 300 & $10^{4}$ & 106 & 300 & $10^{4}$ \\
\hline \multirow{2}{*}{$O(4)$} & $\frac{k_{\varphi}}{\mathrm{MeV}}$ & & & & & & & \\
\hline \hline & 630 & & 143.9 & 91.7 & 83.5 & 124.6 & 99.9 & 91.0 \\
\cline { 2 - 9 } & 700 & & 159.7 & 101.5 & 92.5 & 138.2 & 110.7 & 100.7 \\
\hline \hline \multirow{2}{*}{$U_{L}(2) \times U_{R}(2)$} & 630 & & - & 125.7 & 138.3 & - & - & 138.9 \\
\cline { 2 - 9 } & 700 & & - & 139.6 & 153.5 & - & - & 154.2 \\
\hline
\end{tabular}

Table 1: $f_{\pi}$ in $\mathrm{MeV}$ for various initial conditions at $k_{\varphi}$ and two values of the constituent quark mass.

$k_{\varphi}=630 \mathrm{MeV}$ and the mass term at $k_{\varphi}$ gave $\tilde{\epsilon}_{0}=0.036$. Comparing with fig. 8 we find that this value of $\tilde{\epsilon}_{0}$ is actually too large to induce spontaneous symmetry breaking if the meson fluctuations are taken into account. Given the simplified character of the model considered in [14], however, we find the agreement with the order of magnitude of (8.2) very encouraging. On the other hand, the estimate of $Z_{\varphi}\left(k_{\varphi}\right) \simeq 0.85$ appears to be very inaccurate for the model of [14] and far away from the small values of $Z_{\varphi}\left(k_{\varphi}\right)$ for which the values (8.1), (8.2) were obtained.

We finally compute the chiral condensate from $(2.13)$ with $\bar{h}\left(k_{\varphi}\right)=1$ as

$$
\langle\bar{\psi} \psi\rangle_{0}=\tilde{\epsilon}_{0} Z_{\varphi}^{-\frac{1}{2}}\left(m_{\pi}\right) Z_{\psi}^{-1}\left(k_{\varphi}\right) f_{\pi} k_{\varphi}^{2}
$$

Extracting $Z_{\varphi}\left(m_{\pi}\right)$ from section 4 (fig. 3) we can use this value for a check of the selfconsistency of our scenario. We observe that three different small quantities, $\tilde{\epsilon}_{0}, Z_{\varphi}\left(m_{\pi}\right)$ and $f_{\pi} / k_{\varphi}$ enter here, and it is by far not trivial that a reasonable value of the chiral condensate can be obtained. We normalize the condensate at $k_{\varphi}$ with $Z_{\psi}\left(k_{\varphi}\right)=1$. The result for $\langle\bar{\psi} \psi\rangle_{0}$ as a function of $\tilde{\epsilon}_{0}$ is plotted in fig. 10 . For $k_{\varphi}=630 \mathrm{MeV}, \tilde{\epsilon}_{0}=0.02$ and $h^{2}\left(k_{\varphi}\right)=300$ we obtain in the $O(4)$ model

$$
\left|\langle\bar{\psi} \psi\rangle_{0}\right|^{\frac{1}{3}} \simeq 163 \mathrm{MeV}
$$

We may compare this value with a typical value infered from chiral perturbation theory $[3]$

$$
\left|\langle\bar{\psi} \psi\rangle_{\mathrm{CPT}}\right|^{\frac{1}{3}}(1 \mathrm{GeV}) \simeq(225 \pm 25) \mathrm{MeV} .
$$

This value can be scaled down to $k_{\varphi}=630 \mathrm{MeV}$ by exploiting $k \frac{\partial}{\partial k}\langle\bar{\psi} \psi\rangle_{\mathrm{CPT}}(k) m_{q}(k)=0$. We use here the three-loop $\beta$-function of QCD ( $\overline{\mathrm{MS}}$ scheme) for the running quark mass $[27]$

$$
\frac{k}{m_{q}(k)} \frac{\partial}{\partial k} m_{q}(k)=-\left[\gamma_{1} \frac{\alpha_{s}}{\pi}+\gamma_{2}\left(\frac{\alpha_{s}}{\pi}\right)^{2}+\gamma_{3}\left(\frac{\alpha_{s}}{\pi}\right)^{3}\right]
$$




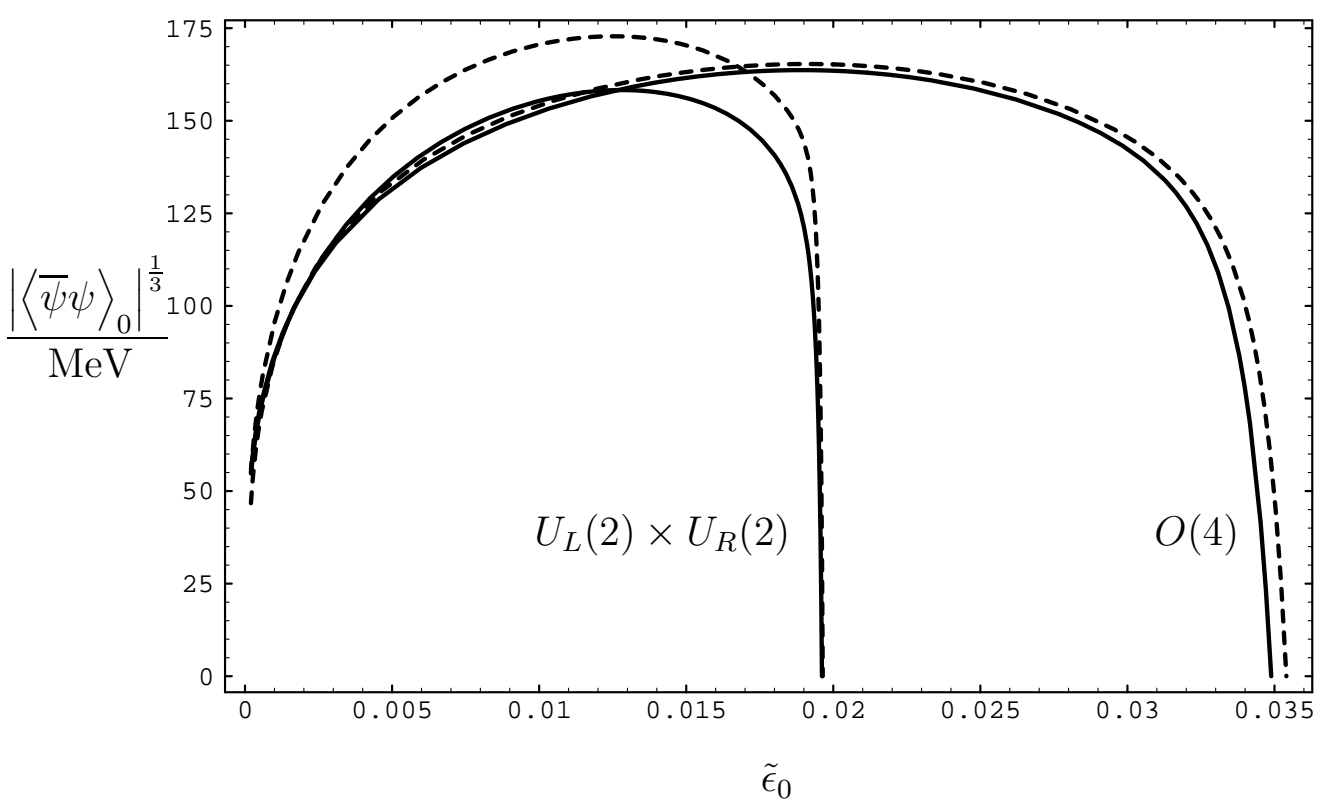

Figure 10: The quark condensate as a function of $\tilde{\epsilon}_{0}$ for $k_{\varphi}=630 \mathrm{MeV}, \lambda_{1}\left(k_{\varphi}\right)=\lambda_{2}\left(k_{\varphi}\right)=0$ and $h^{2}\left(k_{\varphi}\right)=300$ (solid line) as well as $h^{2}\left(k_{\varphi}\right)=10^{4}$ (dashed line).

with

$$
\begin{aligned}
\gamma_{1} & =\frac{3}{2} C_{F} ; \quad \gamma_{2}=\frac{C_{F}}{48}\left[97 N_{c}+9 C_{F}-10 N\right] \\
\gamma_{3} & =\frac{C_{F}}{32}\left[\frac{11413}{108} N_{c}^{2}-\frac{129}{4} N_{c} C_{F}-\left(\frac{278}{27}+24 \zeta(3)\right) N N_{c}+\frac{129}{2} C_{F}^{2}\right. \\
& \left.-(23-24 \zeta(3)) N C_{F}-\frac{35}{27} N^{2}\right]
\end{aligned}
$$

and $C_{F}=\frac{N_{c}^{2}-1}{2 N_{c}}$. This yields

$$
\frac{m_{q}\left(k_{\varphi}\right)}{m_{q}(1 \mathrm{GeV})} \simeq 1.72
$$

for $k_{\varphi}=630 \mathrm{MeV}$ and therefore

$$
\left|\langle\bar{\psi} \psi\rangle_{\mathrm{CPT}}\right|^{\frac{1}{3}}\left(k_{\varphi}\right) \simeq(188 \pm 21) \mathrm{MeV} .
$$

This is in satisfactory agreement with our estimate.

\section{Conclusions}

We have presented here an effective quark-meson model which is supposed to describe the strong interaction dynamics between (constituent) quarks, scalar and pseudo-scalar 
mesons at momentum scales smaller than $k_{\varphi} \simeq 630 \mathrm{MeV}$. The effective average action $\Gamma_{k}$ for this model depends on a scale $k$ which plays the role of an infrared cutoff. The scale dependence of the average action obeys an exact nonperturbative evolution equation. Using a truncation for the general form of $\Gamma_{k}$ this results in approximate flow equations for the meson potential and kinetic term as well as the quark kinetic term and the quarkmeson Yukawa coupling $h$. The initial values of these parameters at the scale $k_{\varphi}$ can, in principle, be computed $[14,15]$ from evolution equations for QCD which are valid for scales larger than $k_{\varphi}$. Following the flow equations from $k_{\varphi}$ to lower scales $k$ one recovers for $k=0$ the effective action, i.e. the generating functional for the $1 P I$ Green functions for the mesons. In particular, the standard nonlinear $\sigma$-model framework of chiral perturbation theory obtains if the expectation value of the meson field is kept fixed at a nonvanishing vacuum expectation value.

We have solved the flow equations numerically and observe how the minimum of the meson potential turns from $\sigma_{0}=0$ at high scales to a nonzero value $\left|\sigma_{0}\right|>0$ for small $k$. The nonvanishing expectation value $\sigma_{0}$ indicates spontaneous chiral symmetry breaking. Our numerical solution allows us to compute the mass scales characteristic for chiral symmetry breaking, i.e. the pion decay constant $f_{\pi}$, the constituent quark mass $m_{q}$ and the chiral condensate $\langle\bar{\psi} \psi\rangle_{0}$. These quantities are computed as functions of the initial values for the scalar mass term $\bar{m}^{2}\left(k_{\varphi}\right)$ and wave function renormalization $Z_{\varphi}\left(k_{\varphi}\right)$. The latter is related to the value of the renormalized Yukawa coupling at $k_{\varphi}$ by $h^{2}\left(k_{\varphi}\right)=Z_{\varphi}^{-1}\left(k_{\varphi}\right)$ once we normalize the quark wave function with $Z_{\psi}\left(k_{\varphi}\right)=1$.

The large ratio between the constituent quark mass $m_{q}$ and $f_{\pi}$ necessitates a large value of the renormalized Yukawa coupling $h_{R}^{2}=h^{2}(k=0)$ according to

$$
h_{R}^{2}=\frac{4 m_{q}^{2}}{f_{\pi}^{2}} \simeq 50 \text {. }
$$

Since $h^{2}(k)$ decreases rapidly with decreasing $k$ we conclude that at the scale $k_{\varphi}$ we have to deal with a strong Yukawa coupling. Our investigation therefore concentrates on large initial values $h^{2}\left(k_{\varphi}\right) \gtrsim 200$. The most crucial observation of the present work is that strong Yukawa couplings imply a very fast running of almost all couplings towards values determined by infrared fixed points or corresponding infrared intervals. The quark-meson model for small scales $k$ therefore looses its memory of the exact initial values of most of the couplings at the scale $k_{\varphi}$. In consequence, strong Yukawa couplings greatly enhance the chances for a reliable estimate of $f_{\pi}$ ! We find that $f_{\pi}$ as well as $m_{q}$ and $\langle\bar{\psi} \psi\rangle_{0}$ depend only on one "relevant" initial value, namely $\tilde{\epsilon}_{0} \sim \bar{m}^{2}\left(k_{\varphi}\right) / k_{\varphi}^{2}$. The value of $h_{R}$ may then be used to fix the required initial value of $\tilde{\epsilon}_{0}$ and therefore to determine $f_{\pi}$ and $\langle\bar{\psi} \psi\rangle_{0}$. For the simplified $O(4)$ model discussed in the present paper we obtain $f_{\pi} \simeq 92 \mathrm{MeV}$ and $\left|\langle\bar{\psi} \psi\rangle_{0}\right|^{\frac{1}{3}} \simeq 163 \mathrm{MeV}$. The comparison with the experimental result $f_{\pi}=93 \mathrm{MeV}$ and the estimate from chiral perturbation theory $\langle\bar{\psi} \psi\rangle_{\mathrm{CPT}}\left(k_{\varphi}\right)=(188 \pm 21) \mathrm{MeV}$ is very encouraging!

Despite the success of the simplified computation of the present work our results have 
partly the character of a feasibility study. Several steps should considerably improve the accuracy of our computation of $f_{\pi}$. First, one should consider the case $N=3$ with a realistic value of $\bar{\nu}$ for the chiral anomaly. This investigation is already prepared in the appendices of the present paper where the central results are exhibited for arbitrary $N$ and $\bar{\nu}$. Second, the explicit chiral symmetry breaking due to current quark masses, in particular the strange quark mass, should be included. Third, the dependence of the Yukawa coupling and the quark kinetic term on the quark momentum can be incorporated. This will provide information on the quark wave function inside the mesons [14].

Furthermore, we have not included residual gluon effects for $k<k_{\varphi}$ in the present work. This would not be necessary if the gluons could completely be integrated out for the determination of the effective quark-meson action at the scale $k_{\varphi}$. Since the latter seems to be a quite difficult task, one may rather use the proposal of ref. [15] and integrate out only the gluons with momenta $q^{2}>k_{\varphi}^{2}$. The remaining gluon fluctuations with momenta $q^{2}<k_{\varphi}^{2}$ give then additional contributions to the flow equations in the quark-meson model. In the context of a computation of $f_{\pi}$ the most important effect seems to be a residual gluonic contribution to $\eta_{\psi}$ and the effective quark meson vertex and therefore to the running of the Yukawa coupling. This can be taken into account by generalizing the average action to include gauge bosons [28] and using the formalism of [15] to integrate them out consecutively. A great part of the gluon fluctuation effects is already included in the contributions from the effective four-fermion interactions for $k>k_{\varphi}$ or from the quark-meson interactions considered in the present paper for $k<k_{\varphi}$. The corrections from residual gluon fluctuations can be found from the explicit formulae in [15]. Since in addition the confinement scale is below the constituent quark mass one may hope that the complicated effects of gluon condensation do not have a very important influence on the determination of $f_{\pi}$.

Finally, a computation of $f_{\pi}$ within QCD, i.e. as a function of $\alpha_{s}(1 \mathrm{GeV})$ and the quark masses, necessitates a reliable computation of $\tilde{\epsilon}_{0}$ and $h^{2}\left(k_{\varphi}\right)$ within the QCD framework for the effective average action proposed in [15]. Thus, the road to an analytical computation of $f_{\pi}\left(\alpha_{s}(1 \mathrm{GeV})\right)$ is still long. Our results should encourage to go it. 


\section{Appendices}

\section{A Linear $\sigma$-model with fermions}

In this appendix we describe the Yukawa couplings of quarks to mesons in the language of the linear $\sigma$-model in a Euclidean formulation. In Euclidean space a Weyl fermion is described by two independent Grassmann variables $\psi_{\alpha}$ and $\bar{\psi}_{\dot{\alpha}}$. (We employ the notation of ref. [22].) We therefore describe the left-handed quarks and their antiparticles, the right-handed antiquarks, by spinor fields $\psi_{L \alpha}$ and $\bar{\psi}_{L \underline{\alpha}}$, the whereas right-handed quarks

and left-handed antiquarks are contained in $\psi_{R}^{\dot{\alpha}}$ and $\bar{\psi}_{R}^{\alpha}$, respectively. In this language a Dirac spinor is composed from left- and right-handed Weyl spinors:

$$
\psi=\left(\begin{array}{c}
\psi_{L \alpha} \\
\psi_{R}^{\dot{\alpha}}
\end{array}\right), \quad \bar{\psi}=\left(\bar{\psi}_{R}^{\alpha}, \bar{\psi}_{L \dot{\alpha}}\right)
$$

The tensors contained in the fermion bilinears are described in the usual way. For example a vector reads

$$
\begin{aligned}
\bar{\psi} \gamma^{m} \psi & =\left(\bar{\psi}_{R}, \bar{\psi}_{L}\right)\left(\begin{array}{cc}
0 & \sigma^{m} \\
\bar{\sigma}^{m} & 0
\end{array}\right)\left(\begin{array}{c}
\psi_{L} \\
\psi_{R}
\end{array}\right) \\
& =\bar{\psi}_{L} \bar{\sigma}^{m} \psi_{L}+\bar{\psi}_{R} \sigma^{m} \psi_{R} .
\end{aligned}
$$

The chiral $S U_{L}(N) \times S U_{R}(N)$ transformations (with $N$ being the number of quark flavors) act independently on the left-- and right-handed quarks:

$$
\begin{gathered}
\psi \rightarrow\left(\begin{array}{cc}
U_{L} & 0 \\
0 & U_{R}
\end{array}\right) \psi \\
\bar{\psi} \rightarrow \bar{\psi}\left(\begin{array}{cc}
U_{R}^{\dagger} & 0 \\
0 & U_{L}^{\dagger}
\end{array}\right)
\end{gathered}
$$

and the $U_{B}(1)$ symmetry corresponding to conserved baryon number acts as

$$
\begin{aligned}
& \psi \rightarrow \exp \left(+\frac{i \vartheta_{B}}{3}\right) \psi \\
& \bar{\psi} \rightarrow \exp \left(-\frac{i \vartheta_{B}}{3}\right) \bar{\psi} .
\end{aligned}
$$

Here $U_{L}$ and $U_{R}$ are $S U(N)$ matrices with $U_{L}^{\dagger} U_{L}=U_{R}^{\dagger} U_{R}=1$. The vector (A.2) and therefore also the kinetic term for the quarks is invariant with respect to these transformations. On the other hand, no mass term is allowed by the symmetry (A.3).

Let us now introduce a complex scalar field $\varphi$ in the $(\overline{\mathbf{N}}, \mathbf{N})$ representation of $S U_{L}(N) \times$ $S U_{R}(N)$ carrying no baryon number. The appropriate flavor transformations are

$$
\begin{aligned}
\varphi & \rightarrow U_{R} \varphi U_{L}^{\dagger} \\
\varphi^{\dagger} & \rightarrow U_{L} \varphi^{\dagger} U_{R}^{\dagger}
\end{aligned}
$$


where $\varphi$ is represented by an $N \times N$ matrix. Its $N^{2}$ complex components should describe mesons or quark-antiquark bilinears. The most general Yukawa coupling between mesons and quarks which is consistent with the $S U_{L}(N) \times S U_{R}(N) \times U_{B}(1)$ flavor symmetry and Osterwalder-Schrader positivity reads ${ }^{8}$

$$
\mathcal{L}_{\mathrm{Yuk}}=\bar{\psi}_{R} \bar{h} \varphi \psi_{L}-\bar{\psi}_{L} \bar{h}^{*} \varphi^{\dagger} \psi_{R}=\bar{\psi}\left(\begin{array}{cc}
\bar{h} \varphi & 0 \\
0 & -\bar{h}^{*} \varphi^{\dagger}
\end{array}\right) \psi
$$

In general, the Yukawa couplings may contain form factors. In a momentum space representation one has

$$
S_{\text {Yuk }}=\Omega \sum_{q, q^{\prime}}\left\{\bar{h}\left(-q, q^{\prime}\right) \bar{\psi}_{R}(q) \varphi\left(q-q^{\prime}\right) \psi_{L}\left(q^{\prime}\right)-\bar{h}^{*}\left(-q, q^{\prime}\right) \bar{\psi}_{L}(q) \varphi^{\dagger}\left(q^{\prime}-q\right) \psi_{R}\left(q^{\prime}\right)\right\}
$$

where we work here on a torus with volume $\Omega$ such that momentum integrals are discrete sums. In particular, this yields the couplings to a constant scalar fields $(\varphi(p)=\varphi \delta(p, 0))$

$$
S_{\text {Yuk }}=\Omega \sum_{q} \bar{\psi}(q)\left(\begin{array}{cc}
\bar{h}(q) \varphi & 0 \\
0 & -\bar{h}^{*}(q) \varphi^{\dagger}
\end{array}\right) \psi(q)
$$

with $\bar{h}(q) \equiv \bar{h}(-q, q)$. We adopt conventions with real Yukawa coupling $\bar{h}$. By a suitable chiral transformation of fermionic phases this is equivalent to the convention with purely imaginary $\bar{h}$ which would be closer to the most commonly used phase convention in a Minkowski space formulation.

By virtue of the $S U_{L}(N) \times S U_{R}(N)$ symmetry we can express the scalar potential $U_{k}(\varphi)$ as a function of a small number of invariants. The number of independent invariants depends on $N$ and we want to classify them. For this purpose it is useful to consider in addition the axial $U_{A}(1)$ transformation

$$
\begin{aligned}
\psi_{L} & \rightarrow \exp \left(+i \vartheta_{A}\right) \psi_{L} \quad, \bar{\psi}_{L} \rightarrow \exp \left(-i \vartheta_{A}\right) \bar{\psi}_{L} \\
\psi_{R} & \rightarrow \exp \left(-i \vartheta_{A}\right) \psi_{R} \quad, \bar{\psi}_{R} \rightarrow \exp \left(+i \vartheta_{A}\right) \bar{\psi}_{R} \\
\varphi & \rightarrow \exp \left(-2 i \vartheta_{A}\right) \varphi \quad, \quad \varphi^{\dagger} \rightarrow \exp \left(+2 i \vartheta_{A}\right) \varphi^{\dagger}
\end{aligned}
$$

(We observe that the Yukawa coupling (A.6) is invariant under $U_{A}(1)$ transformations.) With the help of $S U_{L}(N) \times S U_{R}(N) \times U_{A}(1)$ transformations we can bring $\varphi$ into a standard diagonal form with real nonnegative eigenvalues $\widehat{m}_{a}$. This implies that the $S U_{L}(N) \times S U_{R}(N) \times U_{A}(1)$ invariants can always be expressed in terms of the $N$ eigenvalues $\widehat{m}_{a}^{2}$. There are therefore exactly $N$ independent invariants. Without the $U_{A}(1)$ symmetry the standard form of $\varphi$ is $\exp (i \vartheta) \operatorname{diag}\left(\widehat{m}_{a}\right)$ and the additional phase corresponds to an additional invariant with respect to $S U_{L}(N) \times S U_{R}(N)$ which is not invariant

\footnotetext{
${ }^{8}$ In the conventions of ref. [22] we take $\epsilon=1$ and use $\eta_{L}=\psi_{L}, \tilde{\eta}_{R}=\bar{\psi}_{R}, h_{L R}=h_{R L}=\bar{h}$, $\bar{h}_{L R}^{\prime}=\bar{h}_{R L}^{\prime}=-\bar{h}^{*}, \bar{h}_{R L}=\bar{h}_{L R}=h_{L R}^{\prime}=h_{R L}^{\prime}=0$. In this language the Yukawa couplings $h_{i j}$ are $2 N \times 2 N$ matrices of the type $\left(\begin{array}{cc}0 & \bar{h} \\ \bar{h} & 0\end{array}\right)$ or $\left(\begin{array}{cc}0 & \bar{h}^{\prime} \\ \bar{h}^{\prime} & 0\end{array}\right)$.
} 
under $U_{A}(1)$ transformations. If the symmetry $\varphi \rightarrow \varphi^{\dagger}$ is preserved we may choose for this invariant

$$
\xi=\operatorname{det} \varphi+\operatorname{det} \varphi^{\dagger}
$$

Consider now the $N S U_{L}(N) \times S U_{R}(N) \times U_{A}(1)$ invariants corresponding to the $N$ eigenvalues $\widehat{m}_{a}^{2}$. The first of these invariants is simply the trace

$$
\rho=\operatorname{tr}\left(\varphi^{\dagger} \varphi\right)=\sum_{a} \widehat{m}_{a}^{2} .
$$

For $N>2$ this is the only invariant quadratic in the field $\varphi$ - there is only one singlet in the direct product $(\overline{\mathbf{N}}, \mathbf{N}) \otimes(\mathbf{N}, \overline{\mathbf{N}})$. Only for $N=2$ the invariant $\xi$ is also quadratic in $\varphi$. It corresponds to the singlet in $(\overline{\mathbf{N}}, \mathbf{N}) \otimes(\overline{\mathbf{N}}, \mathbf{N})$. The traceless matrix

$$
\phi=\varphi^{\dagger} \varphi-\frac{1}{N} \rho
$$

can be used to construct higher invariants, i.e.

$$
\tilde{\tau}_{i}=\operatorname{tr} \phi^{i} .
$$

The invariants $\tilde{\tau}_{i}$ can easily be expressed in terms of the $\widehat{m}_{a}^{2}$ using identities of the type

$$
\begin{gathered}
\operatorname{tr} \phi^{2}=\operatorname{tr}\left(\varphi^{\dagger} \varphi\right)^{2}-\frac{1}{N}\left(\operatorname{tr} \varphi^{\dagger} \varphi\right)^{2}=\sum_{a} \widehat{m}_{a}^{4}-\frac{\rho^{2}}{N} \\
\operatorname{tr} \phi^{3}=\sum_{a} \widehat{m}_{a}^{6}-\frac{3}{N} \rho \operatorname{tr} \phi^{2}-\frac{1}{N^{2}} \rho^{3} .
\end{gathered}
$$

For $N=2$ the invariant $\tilde{\tau}_{3}$ can be expressed as a function of $\rho$ and $\tilde{\tau}_{2}$ and similarly for all higher $\tilde{\tau}_{i}$. More generally, we only need to consider the $N$ invariants $\rho, \tilde{\tau}_{2}, \ldots, \tilde{\tau}_{N}$ and find the most general $S U_{L}(N) \times S U_{R}(N)$ symmetric scalar potential

$$
U_{k}(\varphi)=U_{k}\left(\rho, \tilde{\tau}_{2}, \ldots, \tilde{\tau}_{N}, \xi\right) .
$$

\section{B Scalar mass spectrum}

In this appendix we compute the scalar mass spectrum in dependence on the background field for a general form of the potential. We start with an $S U_{L}(N) \times S U_{R}(N) \times U_{A}(1)$ invariant potential. Without loss of generality we can then consider configurations of the type

$$
\varphi_{a b}=\varphi_{a} \delta_{a b}=\widehat{m}_{a} \delta_{a b} .
$$

We parameterize the potential $U_{k}\left(\varphi, \varphi^{\dagger}\right)$ in terms of the invariants $\rho, \tau_{i}$ :

$$
\begin{aligned}
U_{k}\left(\varphi, \varphi^{\dagger}\right) & =U_{k}\left(\rho, \tau_{i}\right) \\
\rho & =\operatorname{tr}\left(\varphi^{\dagger} \varphi\right) \\
\tau_{2} & =\frac{N}{N-1} \tilde{\tau}_{2}=\frac{N}{N-1} \operatorname{tr}\left(\varphi^{\dagger} \varphi-\frac{1}{N} \rho\right)^{2} \\
\tau_{3} & =\frac{N^{2}}{(N-1)(N-2)} \tilde{\tau}_{3}+\tau_{2}^{\frac{3}{2}}
\end{aligned}
$$


whose relation to the eigenvalues $\widehat{m}_{a}^{2}$ can be found in appendix A. (If needed, suitable definitions of $\tau_{i}$ in terms of $\tilde{\tau}_{i}$ have to be chosen for $i \geq 4$.) One obtains for the second derivatives of the potential

$$
\begin{aligned}
\frac{\delta^{2} U_{k}}{\delta \varphi_{R}^{a b} \delta \varphi_{R c d}} & =U_{k}^{\prime} \delta_{a}^{c} \delta_{b}^{d}+2 U_{k}^{\prime \prime} \varphi_{a} \varphi_{c} \delta_{a b} \delta^{c d}+\sum_{i} \frac{\partial U_{k}}{\partial \tau_{i}} \frac{\delta^{2} \tau_{i}}{\delta \varphi_{R}^{a b} \delta \varphi_{R c d}} \\
& +\sqrt{2} \sum_{i} \frac{\partial U_{k}^{\prime}}{\partial \tau_{i}}\left(\varphi_{a} \delta_{a b} \frac{\delta \tau_{i}}{\delta \varphi_{R c d}}+\varphi_{c} \delta^{c d} \frac{\delta \tau_{i}}{\delta \varphi_{R}^{a b}}\right)+\sum_{i, j} \frac{\partial^{2} U_{k}}{\partial \tau_{i} \partial \tau_{j}} \frac{\delta \tau_{j}}{\delta \varphi_{R}^{a b}} \frac{\delta \tau_{i}}{\delta \varphi_{R c d}} \\
\frac{\delta^{2} U_{k}}{\delta \varphi_{I}^{a b} \delta \varphi_{I c d}} & =U_{k}^{\prime} \delta_{a}^{c} \delta_{b}^{d}+\sum_{i} \frac{\partial U_{k}}{\partial \tau_{i}} \frac{\delta^{2} \tau_{i}}{\delta \varphi_{I}^{a b} \delta \varphi_{I c d}} \\
\frac{\delta^{2} U_{k}}{\delta \varphi_{I}^{a b} \delta \varphi_{R c d}} & =0 .
\end{aligned}
$$

Here $\varphi_{a b}=\frac{1}{\sqrt{2}}\left(\varphi_{R a b}+i \varphi_{I a b}\right)$ and primes denote partial derivatives with respect to $\rho$. We observe that $\delta \tau_{i} / \delta \varphi_{I}^{a b}$ vanishes for the configuration (B.1). In order to gain a better understanding of the mass matrix (B.4)-(B.6) we briefly discuss a few special cases:

For the origin at $\varphi_{a}=0$ we are in the symmetric regime and the mass matrix has $2 N^{2}$ real eigenvalues $U_{k}^{\prime}(0, \ldots, 0)$. Here we use the fact that the invariants $\tau_{i}$ are at least quartic in $\varphi$.

If the potential is independent of $\tau_{i}$ it exhibits an enhanced symmetry $S O\left(2 N^{2}\right)$ instead of $S U_{L}(N) \times S U_{R}(N) \times U_{A}(1)$. In case of spontaneous symmetry breaking the minimum of the potential occurs at $U_{k}^{\prime}\left(\rho_{0}\right)=0$. The $N^{2} \times N^{2}$ matrix $\varphi_{a} \varphi_{c} \delta_{a b} \delta^{c d}$ has exactly one eigenvalue $\rho$ whereas all other eigenvalues vanish. Together with the massless fields $\varphi_{I}$ one therefore finds for $\rho=\rho_{0}$ the expected $2 N^{2}-1$ massless Goldstone bosons. The radial excitation has mass squared $2 U_{k}^{\prime \prime}\left(\rho_{0}\right) \rho_{0}$.

We next include the dependence on the invariant $\tau_{2}=\frac{N}{N-1} \tilde{\tau}_{2}=\frac{N}{N-1} \operatorname{tr}\left(\varphi^{\dagger} \varphi\right)^{2}-\frac{1}{N-1} \rho^{2}$. For the configuration (B.1) one has

$$
\begin{aligned}
\frac{\delta \tau_{2}}{\delta \varphi_{R c d}} & =2 \sqrt{2} \delta^{c d}\left(\frac{N}{N-1} \varphi_{c}^{3}-\frac{1}{N-1} \rho \varphi_{c}\right) \\
\frac{\delta^{2} \tau_{2}}{\delta \varphi_{R}^{a b} \delta \varphi_{R c d}} & =2 \delta_{a}^{c} \delta_{b}^{d}\left(\frac{N}{N-1}\left(\varphi_{a}^{2}+\varphi_{b}^{2}\right)-\frac{1}{N-1} \rho\right) \\
& +\frac{2 N}{N-1} \varphi_{a} \varphi_{b} \delta_{a}^{d} \delta_{b}^{c}-\frac{4}{N-1} \varphi_{a} \varphi_{c} \delta_{a b} \delta^{c d} \\
\frac{\delta^{2} \tau_{2}}{\delta \varphi_{I}^{a b} \delta \varphi_{I c d}} & =2 \delta_{a}^{c} \delta_{b}^{d}\left(\frac{N}{N-1}\left(\varphi_{a}^{2}+\varphi_{b}^{2}\right)-\frac{1}{N-1} \rho\right)-\frac{2 N}{N-1} \varphi_{a} \varphi_{b} \delta_{a}^{d} \delta_{b}^{c} .
\end{aligned}
$$

The mass matrix for the fields $\varphi_{I}$ is given by (B.5) and easily evaluated if possible contributions from $\tau_{i}, i>2$ are neglected. The $N$ fields $\varphi_{\text {Iaa }}$ do not mix with $\varphi_{\text {Icd }}, c \neq d$. The corresponding $N$ eigenvalues of the mass matrix are

$$
M_{I a}^{2}=\frac{2}{N-1} \frac{\partial U_{k}}{\partial \tau_{2}}\left(N \varphi_{a}^{2}-\rho\right)+U_{k}^{\prime}
$$


For $a \neq b$ the fields $\varphi_{I a b}$ and $\varphi_{I b a}$ mix, but decouple from $\varphi_{I c d}$ if $c \neq a$ or $b$ or if $d \neq a$ or $b$. There are $N(N-1) / 2$ eigenvalues

$$
\left(M_{I a b}^{-}\right)^{2}=\frac{2}{N-1} \frac{\partial U_{k}}{\partial \tau_{2}}\left[N\left(\varphi_{a}^{2}+\varphi_{b}^{2}+\varphi_{a} \varphi_{b}\right)-\rho\right]+U_{k}^{\prime}
$$

and $N(N-1) / 2$ eigenvalues

$$
\left(M_{I a b}^{+}\right)^{2}=\frac{2}{N-1} \frac{\partial U_{k}}{\partial \tau_{2}}\left[N\left(\varphi_{a}^{2}+\varphi_{b}^{2}-\varphi_{a} \varphi_{b}\right)-\rho\right]+U_{k}^{\prime} .
$$

The discussion for $\varphi_{R}$ is similar. For $a \neq b$ only $\varphi_{R a b}$ and $\varphi_{R b a}$ mix and one finds $N(N-1) / 2$ eigenvalues

$$
\left(M_{R a b}^{+}\right)^{2}=\frac{2}{N-1} \frac{\partial U_{k}}{\partial \tau_{2}}\left[N\left(\varphi_{a}^{2}+\varphi_{b}^{2}+\varphi_{a} \varphi_{b}\right)-\rho\right]+U_{k}^{\prime}
$$

and $N(N-1) / 2$ eigenvalues

$$
\left(M_{R a b}^{-}\right)^{2}=\frac{2}{N-1} \frac{\partial U_{k}}{\partial \tau_{2}}\left[N\left(\varphi_{a}^{2}+\varphi_{b}^{2}-\varphi_{a} \varphi_{b}\right)-\rho\right]+U_{k}^{\prime} .
$$

The mass matrix for the $N$ fields $\varphi_{\text {Raa }}$ reads

$$
\begin{aligned}
\tilde{M}_{R a c}^{2} & =\left[U_{k}^{\prime}+\frac{2}{N-1} \frac{\partial U_{k}}{\partial \tau_{2}}\left(3 N \varphi_{a}^{2}-\rho\right)\right] \delta_{a c} \\
& +2 \varphi_{a} \varphi_{c}\left[U_{k}^{\prime \prime}+\frac{2}{N-1} \frac{\partial U_{k}^{\prime}}{\partial \tau_{2}}\left(N\left(\varphi_{a}^{2}+\varphi_{c}^{2}\right)-2 \rho\right)\right. \\
& \left.+\frac{4}{(N-1)^{2}} \frac{\partial^{2} U_{k}}{\left(\partial \tau_{2}\right)^{2}}\left(N \varphi_{a}^{2}-\rho\right)\left(N \varphi_{c}^{2}-\rho\right)-\frac{2}{N-1} \frac{\partial U_{k}}{\partial \tau_{2}}\right] .
\end{aligned}
$$

Its eigenvalues $M_{\text {Rac }}^{2}$ have, in general, no particularly simple form.

If we specialize to $N$ equal values $\varphi_{a}^{2}=\frac{1}{N} \rho$ the matrix $\tilde{M}_{R}^{2}$ simplifies considerably:

$$
\tilde{M}_{R a c}^{2}=\left(U_{k}^{\prime}+\frac{4 \rho}{N-1} \frac{\partial U_{k}}{\partial \tau_{2}}\right) \delta_{a c}+\left(2 U_{k}^{\prime \prime}-\frac{4}{N-1} \frac{\partial U_{k}}{\partial \tau_{2}}\right) \varphi_{a} \varphi_{c} .
$$

This matrix has $(N-1)$ eigenvalues

$$
\left(M_{R}^{0}\right)^{2}=U_{k}^{\prime}+\frac{4 \rho}{N-1} \frac{\partial U_{k}}{\partial \tau_{2}}
$$

and one eigenvalue

$$
\left(M_{R}^{R}\right)^{2}=U_{k}^{\prime}+2 U_{k}^{\prime \prime} \rho
$$

For this special case one finds

$$
\left(M_{\text {Iab }}^{-}\right)^{2}=\left(M_{\text {Rab }}^{+}\right)^{2}=\left(M_{R}^{0}\right)^{2}
$$


and

$$
M_{I a}^{2}=\left(M_{I a b}^{+}\right)^{2}=\left(M_{R a b}^{-}\right)^{2}=U_{k}^{\prime} .
$$

For $U_{k}^{\prime}=0$ the $N^{2}$ massless Goldstone bosons (B.20) correspond to the symmetry breaking $S U_{L}(N) \times S U_{R}(N) \times U_{A}(1) \rightarrow S U(N)$ where the unbroken $S U(N)$ is the diagonal subgroup of $S U_{L}(N) \times S U_{R}(N)$. In addition we have $N^{2}-1$ massive scalars (B.19) in the adjoint representation of $S U(N)$. For $U_{k}^{\prime}\left(\rho_{0}\right)=0$ their mass terms are positive provided $\partial U_{k} / \partial \tau_{2} \geq 0$. Finally there is a singlet with mass term $2 U_{k}^{\prime \prime}\left(\rho_{0}\right) \rho_{0}$.

Another interesting special case occurs for $\varphi_{a}^{2}=\rho \delta_{a 1}$ which corresponds to the symmetry breaking $S U_{L}(N) \times S U_{R}(N) \times U_{A}(1) \rightarrow S U_{L}(N-1) \times S U_{R}(N-1) \times U(1) \times U(1)$. The eigenvalues $M_{I a}^{2}$ contain $N-1$ values $U_{k}^{\prime}-\frac{2 \rho}{N-1} \frac{\partial U_{k}}{\partial \tau_{2}}$ and one value $U_{k}^{\prime}+2 \rho \frac{\partial U_{k}}{\partial \tau_{2}}$. The eigenvalues $\left(M_{I a b}^{+}\right)^{2},\left(M_{I a b}^{-}\right)^{2},\left(M_{R a b}^{+}\right)^{2}$ and $\left(M_{R a b}^{-}\right)^{2}$ decompose each into $(N-1)(N-2) / 2$ values $U_{k}^{\prime}-\frac{2 \rho}{N-1} \frac{\partial U_{k}}{\partial \tau_{2}}$ and $N-1$ values $U_{k}^{\prime}+2 \rho \frac{\partial U_{k}}{\partial \tau_{2}}$. Finally $\tilde{M}_{R a c}^{2}$ has $N-1$ eigenvalues $U_{k}^{\prime}-\frac{2 \rho}{N-1} \frac{\partial U_{k}}{\partial \tau_{2}}$ and one eigenvalue $U_{k}^{\prime}+2 \rho U_{k}^{\prime \prime}+6 \rho \frac{\partial U_{k}}{\partial \tau_{2}}+8 \rho^{2} \frac{\partial U_{k}^{\prime}}{\partial \tau_{2}}+8 \rho^{3} \frac{\partial^{2} U_{k}}{\left(\partial \tau_{2}\right)^{2}}$. For $U_{k}^{\prime}+2 \rho \frac{\partial U_{k}}{\partial \tau_{2}}=0$ we observe the expected $4 N-3$ massless Goldstone bosons.

Next we should take into account that the axial $U_{A}(1)$ symmetry is broken due to anomalies. The most general potential can therefore also depend on

$$
\xi=\operatorname{det} \varphi+\operatorname{det} \varphi^{\dagger}
$$

We observe that $\xi$ violates $U_{A}(1)$ but is invariant under $S U_{L}(N) \times S U_{R}(N)$. The second possible $U_{A}(1)$ violating $S U_{L}(N) \times S U_{R}(N)$ invariant, $\omega=i\left(\operatorname{det} \varphi-\operatorname{det} \varphi^{\dagger}\right)$, violates the discrete symmetry $\varphi \rightarrow \varphi^{\dagger}$ and hence the $C P$ invariance of the model and will therefore be discarded here ${ }^{9}$. The configuration (B.1) with $N$ real $\varphi_{a}$ is not the most general configuration in this case. An overall phase for all $\varphi_{a}$ cannot be removed anymore by $U_{A}(1)$ transformations. In contrast to the invariants $\rho$ and $\tau_{i}$ the properties of $\xi$ depend crucially on $N$ : $\xi$ is of order $\varphi^{N}$. In addition there is an important difference between $N$ even and odd. For $N$ even the discrete symmetry $\varphi \rightarrow-\varphi$ is part of $S U_{L}(N) \times S U_{R}(N)$ and is therefore respected by $\xi$. For $N$ odd $\xi$ is not invariant under this discrete symmetry. We also note that all invariants observe the discrete symmetry $\varphi \rightarrow \varphi^{T}$ and therefore also $\varphi \rightarrow \varphi^{*}$.

We restrict the discussion here to scalar field configurations with real diagonal eigenvalues $\widehat{m}_{a}$ which are sufficient for deriving the flow equations for all derivatives of $U_{k}$ with respect to $\rho$ and the $\tau_{i}$ (but not $\xi$ ). The discrete symmetry $\varphi \rightarrow \varphi^{*}$ of the potential implies invariance under $\varphi_{I a b} \rightarrow-\varphi_{I a b}$. For real $\widehat{m}_{a}$ we can choose without further loss of generality the configuration (B.1) which respects $\varphi_{I} \rightarrow-\varphi_{I}$. As an immediate consequence the mass matrix does not mix $\varphi_{R}$ and $\varphi_{I}$ and (B.6) remains true. The mass matrices (B.4) and (B.5) acquire additional contributions:

$$
\Delta \frac{\delta^{2} U_{k}}{\delta \varphi_{R}^{a b} \delta \varphi_{R c d}}=\sqrt{2} \frac{\partial U_{k}^{\prime}}{\partial \xi}\left(\varphi_{a} \delta_{a b} \frac{\delta \xi}{\delta \varphi_{R c d}}+\varphi_{c} \delta^{c d} \frac{\delta \xi}{\delta \varphi_{R}^{a b}}\right)
$$

\footnotetext{
${ }^{9}$ We note that the sum $\omega^{2}+\xi^{2}$ is proportional to $\operatorname{det} \varphi \operatorname{det} \varphi^{\dagger}$ and can therefore be expressed in terms of the invariants $\rho, \tau_{i}$.
} 


$$
\begin{aligned}
& +\sum_{i} \frac{\partial^{2} U_{k}}{\partial \xi \partial \tau_{i}}\left(\frac{\delta \xi}{\delta \varphi_{R c d}} \frac{\delta \tau_{i}}{\delta \varphi_{R}^{a b}}+\frac{\delta \xi}{\delta \varphi_{R}^{a b}} \frac{\delta \tau_{i}}{\delta \varphi_{R c d}}\right) \\
& +\frac{\partial^{2} U_{k}}{(\partial \xi)^{2}} \frac{\delta \xi}{\delta \varphi_{R}^{a b}} \frac{\delta \xi}{\delta \varphi_{R c d}}+\frac{\partial U_{k}}{\partial \xi} \frac{\delta^{2} \xi}{\delta \varphi_{R}^{a b} \delta \varphi_{R c d}} \\
\Delta \frac{\delta^{2} U_{k}}{\delta \varphi_{I}^{a b} \delta \varphi_{I c d}} & =\frac{\partial U_{k}}{\partial \xi} \frac{\delta^{2} \xi}{\delta \varphi_{I}^{a b} \delta \varphi_{I c d}} .
\end{aligned}
$$

Here we have used $\delta \xi / \delta \varphi_{I}=0$ for the configuration (B.1). Writing

$$
\xi=\frac{1}{N !} \epsilon^{a_{1} \ldots a_{N}} \epsilon^{b_{1} \ldots b_{N}}\left(\varphi_{a_{1} b_{1}} \ldots \varphi_{a_{N} b_{N}}+\varphi_{a_{1} b_{1}}^{*} \ldots \varphi_{a_{N} b_{N}}^{*}\right)
$$

one has

$$
\begin{aligned}
\frac{\delta \xi}{\delta \varphi_{R c d}} & =\frac{1}{\sqrt{2}}\left(\frac{\delta \xi}{\delta \varphi_{c d}}+\frac{\delta \xi}{\delta \varphi_{c d}^{*}}\right) \\
\frac{\delta \xi}{\delta \varphi_{I c d}} & =\frac{i}{\sqrt{2}}\left(\frac{\delta \xi}{\delta \varphi_{c d}}-\frac{\delta \xi}{\delta \varphi_{c d}^{*}}\right)
\end{aligned}
$$

with

$$
\begin{aligned}
\frac{\delta \xi}{\delta \varphi_{c d}} & =\frac{1}{(N-1) !} \sum_{a_{2} \ldots a_{N}} \epsilon^{c a_{2} \ldots a_{N}} \epsilon^{d a_{2} \ldots a_{N}} \varphi_{a_{2}} \ldots \varphi_{a_{N}} \\
\frac{\delta \xi}{\delta \varphi_{c d}^{*}} & =\frac{1}{(N-1) !} \sum_{a_{2} \ldots a_{N}} \epsilon^{c a_{2} \ldots a_{N}} \epsilon^{d a_{2} \ldots a_{N}} \varphi_{a_{2}}^{*} \ldots \varphi_{a_{N}}^{*}
\end{aligned}
$$

for a diagonal configuration $\varphi$. Taking into account the reality of $\varphi$ in (B.1) one recovers $\delta \xi / \delta \varphi_{I}=0$ and

$$
\frac{\delta \xi}{\delta \varphi_{R c d}}=\sqrt{2} \delta^{c d} \prod_{a_{i} \neq c} \varphi_{a_{i}} .
$$

For the particular configuration where all $\varphi_{a}^{2}$ equal $\rho / N$ one finds

$$
\frac{\delta \xi}{\delta \varphi_{R c d}}=\sqrt{2}\left(\frac{\rho}{N}\right)^{\frac{N-1}{2}} \delta^{c d}
$$

and similarly

$$
\frac{\delta^{2} \xi}{\delta \varphi_{R}^{a b} \delta \varphi_{R}^{c d}}=-\frac{\delta^{2} \xi}{\delta \varphi_{I}^{a b} \delta \varphi_{I}^{c d}}=\frac{1}{(N-2) !} \sum_{e_{3} \ldots e_{N}} \epsilon_{a c e_{3} \ldots e_{N}} \epsilon_{b d e_{3} \ldots e_{N}} \varphi^{e_{3}} \ldots \varphi^{e_{N}}
$$

or

$$
\begin{aligned}
& \frac{\delta^{2} \xi}{\delta \varphi_{R}^{a a} \delta \varphi_{R}^{c c}}=\left(1-\delta_{a c}\right) \prod_{e_{i} \neq a, c} \varphi_{e_{i}} \\
& \frac{\delta^{2} \xi}{\delta \varphi_{R}^{a b} \delta \varphi_{R}^{b a}}=-\left(1-\delta_{a b}\right) \prod_{e_{i} \neq a, b} \varphi_{e_{i}} .
\end{aligned}
$$


In the following we will specialize our discussion to the particular configuration ${ }^{10}$

$$
\varphi_{a b}=\left(\frac{\rho}{N}\right)^{\frac{1}{2}} \delta_{a b}
$$

We restrict the discussion here to a potential linear in $\xi$ such that $\partial U_{k}^{\prime} / \partial \xi=\partial^{2} U_{k} / \partial \xi \partial \tau_{2}=$ $\partial^{2} U_{k} / \partial \xi^{2}=0$. The terms $\sim \delta \xi / \delta \varphi_{R}$ vanish in this case and the second functional derivatives read

$$
\frac{\delta^{2} \xi}{\delta \varphi_{R}^{a a} \delta \varphi_{R}^{c c}}=-\frac{\delta^{2} \xi}{\delta \varphi_{I}^{a a} \delta \varphi_{I}^{c c}}=\left(\frac{\rho}{N}\right)^{\frac{N-2}{2}}\left(1-\delta_{a c}\right)
$$

and for $a \neq b$

$$
\frac{\delta^{2} \xi}{\delta \varphi_{R}^{a b} \delta \varphi_{R}^{c d}}=-\frac{\delta^{2} \xi}{\delta \varphi_{I}^{a b} \delta \varphi_{I}^{c d}}=-\left(\frac{\rho}{N}\right)^{\frac{N-2}{2}} \delta_{a d} \delta_{b c} .
$$

The mass matrices for the diagonal and off-diagonal $\varphi_{a b}$ as well as for $\varphi_{R}$ and $\varphi_{I}$ remain decoupled. For the diagonal part one finds

$$
\begin{aligned}
\tilde{M}_{I a c}^{2} & =U_{k}^{\prime} \delta_{a c} \\
\tilde{M}_{R a c}^{2} & =\left(U_{k}^{\prime}+\frac{4 \rho}{N-1} \frac{\partial U_{k}}{\partial \tau_{2}}-\left(\frac{\rho}{N}\right)^{\frac{N-2}{2}} \frac{\partial U_{k}}{\partial \xi}\right) \delta_{a c} \\
& +\left(2 U_{k}^{\prime \prime}-\frac{4}{N-1} \frac{\partial U_{k}}{\partial \tau_{2}}+\left(\frac{\rho}{N}\right)^{\frac{N-4}{2}} \frac{\partial U_{k}}{\partial \xi}\right) \frac{\rho}{N}
\end{aligned}
$$

The matrix $\tilde{M}_{I}^{2}$ has $N-1$ eigenvalues

$$
\left(M_{I}^{0}\right)^{2}=U_{k}^{\prime}+\left(\frac{\rho}{N}\right)^{\frac{N-2}{2}} \frac{\partial U_{k}}{\partial \xi}
$$

and one eigenvalue

$$
\left(M_{I}^{R}\right)^{2}=U_{k}^{\prime}-(N-1)\left(\frac{\rho}{N}\right)^{\frac{N-2}{2}} \frac{\partial U_{k}}{\partial \xi}
$$

whereas $\tilde{M}_{R}^{2}$ has $N-1$ eigenvalues

$$
\left(M_{R}^{0}\right)^{2}=U_{k}^{\prime}+\frac{4 \rho}{N-1} \frac{\partial U_{k}}{\partial \tau_{2}}-\left(\frac{\rho}{N}\right)^{\frac{N-2}{2}} \frac{\partial U_{k}}{\partial \xi}
$$

and one eigenvalue

$$
\left(M_{R}^{R}\right)^{2}=U_{k}^{\prime}+2 U_{k}^{\prime \prime} \rho+(N-1)\left(\frac{\rho}{N}\right)^{\frac{N-2}{2}} \frac{\partial U_{k}}{\partial \xi} .
$$

\footnotetext{
${ }^{10}$ The reader should not get confused by our use of the symbol $\rho$ for two different purposes - once for the invariant $\rho \equiv \tau_{1}$ and also for the field configuration $\rho=N \sigma^{2}$. Quantities like $U_{k}^{\prime}$ always denote derivatives with respect to $\tau_{1}$ at fixed $\tau_{2}$ and $\xi$. On the other hand, for the configuration (B.31) the invariant $\xi$ becomes a nonvanishing function of $\sigma^{2}=\rho / N$.
} 
The mass eigenvalues for the off-diagonal fields are

$$
\begin{aligned}
& \left(M_{\text {Iab }}^{-}\right)^{2}=U_{k}^{\prime}+\frac{4 \rho}{N-1} \frac{\partial U_{k}}{\partial \tau_{2}}-\left(\frac{\rho}{N}\right)^{\frac{N-2}{2}} \frac{\partial U_{k}}{\partial \xi} \\
& \left(M_{I a b}^{+}\right)^{2}=U_{k}^{\prime}+\left(\frac{\rho}{N}\right)^{\frac{N-2}{2}} \frac{\partial U_{k}}{\partial \xi} \\
& \left(M_{\text {Rab }}^{+}\right)^{2}=U_{k}^{\prime}+\frac{4 \rho}{N-1} \frac{\partial U_{k}}{\partial \tau_{2}}-\left(\frac{\rho}{N}\right)^{\frac{N-2}{2}} \frac{\partial U_{k}}{\partial \xi} \\
& \left(M_{\text {Rab }}^{-}\right)^{2}=U_{k}^{\prime}+\left(\frac{\rho}{N}\right)^{\frac{N-2}{2}} \frac{\partial U_{k}}{\partial \xi} .
\end{aligned}
$$

At the potential minimum one has $U_{k}^{\prime}+\left(\frac{\rho_{0}}{N}\right)^{\frac{N-2}{2}} \frac{\partial U_{k}}{\partial \xi}=0$ and we observe $N^{2}-1$ Goldstone bosons ("pions") (B.36), (B.41), (B.43), one less than for $\partial U_{k} / \partial \xi=0$. The scalar which has acquired a mass due to the $U_{A}(1)$ violating term in the potential - the " $\eta^{\prime}-$ meson" - has positive mass squared (with $\left.\partial U_{k} / \partial \xi \leq 0\right)$, (B.37):

$$
M_{\xi}^{2}=-N\left(\frac{\rho_{0}}{N}\right)^{\frac{N-2}{2}} \frac{\partial U_{k}}{\partial \xi}\left(\rho_{0}, \tau_{i}=0, \xi=2\left(\frac{\rho_{0}}{N}\right)^{\frac{N-2}{2}}\right) .
$$

There are also $N^{2}-1$ scalars in the adjoint representation of the unbroken diagonal $S U(N)$ which have mass squared $\left(M_{R}^{0}\right)^{2},($ B.38), (B.40), (B.42):

$$
\left(M_{R}^{0}\right)^{2}=M_{\tau}^{2}+\frac{2}{N} M_{\xi}^{2}, \quad M_{\tau}^{2}=\frac{4 \rho_{0}}{N-1} \frac{\partial U_{k}}{\partial \tau_{2}}\left(\rho_{0}, \tau_{i}=0, \xi=2\left(\frac{\rho_{0}}{N}\right)^{\frac{N-2}{2}}\right) .
$$

Finally, there is the singlet ("radial mode" or " $\sigma$-field") with mass given by (B.39).

We observe for $\rho=0$ that $M_{\tau}^{2}$ vanishes and $M_{\xi}^{2}$ vanishes for $N>2$. In the symmetric regime one therefore has $2 N^{2}$ real scalar fields with mass squared $U_{k}^{\prime}(0)$. This is different for $N=2$ where $M_{\xi}^{2}(0)=-2 \frac{\partial U_{k}}{\partial \xi}(0)$. In the symmetric regime the complex $(\mathbf{2}, \mathbf{2})$ representation decays in this particular case into two irreducible (real) representations with mass squared $U_{k}^{\prime}(0)+\frac{\partial U_{k}}{\partial \xi}(0)$ and $U_{k}^{\prime}(0)-\frac{\partial U_{k}}{\partial \xi}(0)$. In an obvious notation with Pauli matrices $\tau_{k}$ we can write the complex $(\mathbf{2}, \mathbf{2})$ representation $\varphi$ as

$$
\varphi=\frac{1}{2}\left(\sigma-i \eta^{\prime}\right)+\frac{1}{2}\left(a^{k}+i \pi^{k}\right) \tau_{k}
$$

The fields $\left(\sigma, \pi^{k}\right)$ form an irreducible representation - they represent the standard linear $\sigma-$ model [4] with scalars in a real four-component vector representation. In the standard linear $\sigma$-model the fields $\left(\eta^{\prime}, a^{k}\right)$ are omitted ${ }^{11}$ and the scalar potential can be expressed

\footnotetext{
${ }^{11}$ This is a self-consistent truncation for $N=2$, since $\left(\eta^{\prime}, a^{k}\right)$ form a separate irreducible representation.
} 
with the help of only one invariant $\rho=\frac{1}{2}\left(\sigma^{2}+\pi^{k} \pi_{k}\right)$. In terms of $\left(\sigma, \pi^{k}\right)$ the Yukawa coupling (A.6) with imaginary $\bar{h}$ reads

$$
\begin{array}{r}
\mathcal{L}_{\text {Yuk }}=i \bar{h}_{\sigma} \bar{\psi}\left(\sigma+i \pi^{k} \tau_{k} \bar{\gamma}\right) \psi \\
\bar{h}=2 i \bar{h}_{\sigma}, \quad \bar{h}_{\sigma}=\bar{h}_{\sigma}^{*}
\end{array}
$$

with $\bar{\gamma}$ being the Euclidean analog of $\gamma^{5}$.

If we decide to include the fields $\left(\eta^{\prime}, a^{k}\right)$ we obtain for the invariants $\rho, \tau_{2}$ and $\xi$

$$
\begin{aligned}
\rho & =\frac{1}{2}\left(\pi^{a} \pi_{a}+a^{a} a_{a}\right) \\
\tau_{2} & =\left(a_{a} a^{a}\right)\left(\pi_{b} \pi^{b}\right)-\left(a_{a} \pi^{a}\right)\left(a_{b} \pi^{b}\right) \\
\xi & =\frac{1}{2}\left(\pi^{a} \pi_{a}-a^{a} a_{a}\right) .
\end{aligned}
$$

Here we have defined four-vectors $\pi_{a}=\left(\pi_{k}, \sigma\right)$ and $a_{a}=\left(a_{k}, \eta^{\prime}\right)$. We observe that all invariants in (B.48) are also invariant under the discrete symmetry $a \rightarrow-a$ which is equivalent to $\varphi \rightarrow \varphi^{\dagger}$. The invariant violating this symmetry is $\omega=i\left(\operatorname{det} \varphi-\operatorname{det} \varphi^{\dagger}\right) \sim$ $a^{a} \pi_{a}$. The quartic invariant $\tau_{2}$ can be constructed from $\rho, \xi$ and $\omega$.

\section{Evolution equation for $\bar{\lambda}_{2}$}

In this appendix we discuss in more detail the $\beta$ function for $\bar{\lambda}_{2}$ in the SSB regime. The evolution equation (3.22) can formally be written as

$$
\begin{aligned}
\frac{\partial}{\partial t} \bar{\lambda}_{2} & =-\frac{1}{2} \int \frac{d^{d} q}{(2 \pi)^{d}} \frac{\widehat{\partial}}{\partial t}\left\{\frac{N^{2}}{4} \frac{\bar{\lambda}_{2}^{2}}{\left(Z_{\varphi} P\right)^{2}}\right. \\
& +\frac{9\left(N^{2}-4\right)}{4} \frac{\bar{\lambda}_{2}^{2}}{\left(Z_{\varphi} P+\bar{\lambda}_{2} \rho_{0}\right)^{2}}+\frac{N^{2} \bar{\lambda}_{2}}{2 \rho_{0}}\left[\frac{1}{Z_{\varphi} P+\bar{\lambda}_{2} \rho_{0}}-\frac{1}{Z_{\varphi} P}\right] \\
& \left.+\frac{6 \bar{\lambda}_{2}\left(\frac{1}{4} \bar{\lambda}_{2}+\bar{\lambda}_{1}\right)}{\rho_{0}\left(\frac{1}{2} \bar{\lambda}_{2}-\bar{\lambda}_{1}\right)}\left[\frac{1}{Z_{\varphi} P+2 \bar{\lambda}_{1} \rho_{0}}-\frac{1}{Z_{\varphi} P+\bar{\lambda}_{2} \rho_{0}}\right]\right\}
\end{aligned}
$$

where $\frac{\widehat{\partial}}{\partial t}$ acts only on the infrared cutoff in $P$, i.e. $\frac{\widehat{\partial}}{\partial t}=\frac{1}{Z_{\varphi}} \frac{\partial R_{k}}{\partial t} \frac{\partial}{\partial P}$. Alternatively, we may use the form

$$
\begin{aligned}
\frac{\partial}{\partial t} \bar{\lambda}_{2} & =-\frac{1}{2} \int \frac{d^{d} q}{(2 \pi)^{d}} \frac{\widehat{\partial}}{\partial t}\left\{\frac{N^{2}}{4} \frac{\bar{\lambda}_{2}^{2}}{\left(Z_{\varphi} P\right)^{2}}+\frac{9\left(N^{2}-4\right)}{4} \frac{\bar{\lambda}_{2}^{2}}{\left(Z_{\varphi} P+\bar{\lambda}_{2} \rho_{0}\right)^{2}}\right. \\
& \left.-\frac{N^{2}}{2} \frac{\bar{\lambda}_{2}^{2}}{\left(Z_{\varphi} P\right)\left(Z_{\varphi} P+\bar{\lambda}_{2} \rho_{0}\right)}+\frac{3 \bar{\lambda}_{2}^{2}+12 \bar{\lambda}_{1} \bar{\lambda}_{2}}{\left(Z_{\varphi} P+2 \bar{\lambda}_{1} \rho_{0}\right)\left(Z_{\varphi} P+\bar{\lambda}_{2} \rho_{0}\right)}\right\}
\end{aligned}
$$


which is close to the $t$-derivative $\frac{\widehat{\partial}}{\partial t}$ of the contribution from the perturbative one-loop graphs, with $\rho_{0}$-dependent masses and the infrared cutoff in the propagator taken into account. For $d=4, \rho_{0} \rightarrow 0, P=q^{2}$ and omitting the $t$-derivatives one recognizes the one-loop correction for $\bar{\lambda}_{2}[16,17]$.

One may ask to what extent the $\beta$ function for $\bar{\lambda}_{2}$ depends on the choice of the configuration (3.13). For this purpose we have also evaluated $\partial U_{k} / \partial t$ for a configuration

$$
\varphi_{1}^{2}=\frac{\rho_{0}}{N}+\epsilon, \quad \varphi_{2}^{2}=\frac{\rho_{0}}{N}-\epsilon, \quad \varphi_{a}^{2}=\frac{\rho_{0}}{N} \text { for } a \geq 3
$$

with infinitesimal $\epsilon$ and

$$
\tau_{2}=\frac{2 N}{N-1} \epsilon^{2}
$$

From the contribution proportional to $\epsilon^{2}$ in $\partial U_{k} / \partial t$ one obtains the flow equation

$$
\begin{aligned}
\frac{\partial}{\partial t} \widehat{\lambda}_{2} & =-\frac{1}{2} \int \frac{d^{d} q}{(2 \pi)^{d}} \frac{\widehat{\partial}}{\partial t}\left\{\frac{N(2 N-3)}{2} \frac{\bar{\lambda}_{2}^{2}}{\left(Z_{\varphi} P\right)^{2}}+\frac{\left(2 N^{2}+5 N-18\right)}{2} \frac{\bar{\lambda}_{2}^{2}}{\left(Z_{\varphi} P+\bar{\lambda}_{2} \rho_{0}\right)^{2}}\right. \\
& \left.-\frac{N \bar{\lambda}_{2}^{2}}{\left(Z_{\varphi} P\right)\left(Z_{\varphi} P+\bar{\lambda}_{2} \rho_{0}\right)}+\frac{3 \bar{\lambda}_{2}^{2}+12 \bar{\lambda}_{1} \bar{\lambda}_{2}}{\left(Z_{\varphi} P+2 \bar{\lambda}_{1} \rho_{0}\right)\left(Z_{\varphi} P+\bar{\lambda}_{2} \rho_{0}\right)}\right\} .
\end{aligned}
$$

We observe that for $N=2$ the configuration (C.3) is equivalent to (3.13) and the evolution equations for $\bar{\lambda}_{2}$ and $\widehat{\lambda}_{2},(\mathrm{C} .2)$ and (C.5), respectively, agree. For $N \geq 3$, however, the two equations are not identical and this is the reason why we have chosen the symbol $\widehat{\lambda}_{2}$ in (C.5). The difference is related to the truncated higher invariants $\tau_{i}, i \geq 3$. The validity of the evolution equation (C.2) corresponds to a definition of the higher invariants where all $\tau_{i}$ vanish for $i \geq 3$ for the configuration (3.13). Inserting (3.13) into (A.13) we find $\tilde{\tau}_{3}=-\frac{(N-1)(N-2)}{N^{2}}\left(\tau_{2}\right)^{\frac{3}{2}}$ and therefore (B.3) $\tau_{3}=0$. On the other hand, we find for the configuration (C.3) that $\tilde{\tau}_{3}$ vanishes (but not $\tilde{\tau}_{4}$ ) which leads to

$$
\tau_{3}=\left(\tau_{2}\right)^{3 / 2}
$$

A truncation is well defined only once we specify the exact definition of the higher invariants which we omit. This gives then a unique definition of the running of coupling constants. For $N \geq 3$ the difference in the results for $k \rightarrow 0$ between the use of (C.2) or (C.5) can be taken as a rough estimate of the uncertainty due to the truncation of the higher invariants.

\section{The infrared cutoff for fermions}

The choice of the infrared cutoff for fermions is not completely obvious for various reasons. First of all, chiral fermions do not allow a mass term. Since we want to remain consistent with chiral symmetries (a necessity for neutrinos, for example), the infrared cutoff must have the same Lorentz structure as the kinetic term, i.e. $R_{k F} \sim \gamma^{\mu} q_{\mu}[22]$. On the 
other hand, for $q^{2} \rightarrow 0$ the infrared cutoff should be $\sim k$, e.g. $R_{k F} \sim k \phi / \sqrt{q^{2}}$. The nonanalyticity of $\sqrt{q^{2}}$ may then be a cause of problems. We will develop in this appendix a few criteria for a reasonable infrared cutoff and finally propose one which seems suitable for practical calculations.

First of all, the fermionic infrared cutoff term $\Delta_{k} S_{F}$ should be quadratic in the fermion fields. We next require that $\Delta_{k} S_{F}$ should respect all symmetries of the kinetic term for free fermions. This includes chiral symmetries and Lorentz invariance. (Gauge symmetries may be implemented by covariant derivatives in a background gauge field [28] but this if of no concern in the present paper.) The symmetry requirement implies in a Fourier representation

$$
\Delta_{k} S_{F}=\Omega \sum_{q} \bar{\eta}_{\dot{\alpha}}(q) Z_{\psi}(k)(\not q)_{\alpha}^{\dot{\alpha}} r_{F}\left(\frac{q^{2}}{k^{2}}\right) \eta^{\alpha}(q) \equiv \Omega \bar{\eta} Z_{\psi} \phi q r_{F} \eta
$$

where $\bar{\eta}, \eta$ are Weyl spinors, $\alpha, \dot{\alpha}$ denote spinor indices, $\not q=q_{\mu} \bar{\sigma}^{\mu}$ and $\bar{\sigma}^{\mu}$ is the restriction of $\gamma^{\mu}$ for left-handed Weyl spinors (with a suitable choice for right-handed Weyl spinors). We also have omitted possible internal indices labeling different Weyl spinors (for conventions see [22]). The wave function renormalization $Z_{\psi}$ is chosen for convenience such that it matches with an approximation for the fermion kinetic term $\Omega \bar{\eta} Z_{\psi} \phi \eta$ in $\Gamma_{k}$. (Typically $Z_{\psi}$ is diagonal in the internal indices $i, j$, but different fermion species may have different wave function renormalization constants.) The third condition requires that $\Delta_{k} S_{F}$ acts effectively as an infrared cutoff. This means that for $k \rightarrow \infty$ the combination $Z_{\psi} r_{F}\left(\frac{q^{2}}{k^{2}}\right)$ should diverge for all values of $q^{2}$. This divergence should also occur for finite $k$ and $q^{2} / k^{2} \rightarrow 0$ and be at least as strong as $\left(k^{2} / q^{2}\right)^{1 / 2}$. As a fourth point we remark that $\Gamma_{k}$ becomes the effective action in the limit $k \rightarrow 0$ only if $\lim _{k \rightarrow 0} \Delta_{k} S_{F}=0$. This should hold for all Fourier modes separately, i.e. for $\lim _{k \rightarrow 0} Z_{\psi}(k)=$ const. one requires

$$
\lim _{k \rightarrow 0} r_{F}\left(\frac{q^{2}}{k^{2}}\right) \not q=0 \text {. }
$$

Even though on a torus with longest circumference $L$ the minimum value $q_{\min }^{2}=\pi^{2} / L^{2}$ does not vanish, we want a smooth limit to infinite volume and request that (D.2) also holds in the limit $q^{2} \rightarrow 0$. Together with the third condition this implies exactly

$$
\lim _{q^{2} / k^{2} \rightarrow 0} r_{F}\left(\frac{q^{2}}{k^{2}}\right) \sim\left(\frac{q^{2}}{k^{2}}\right)^{-\frac{1}{2}} .
$$

The requirement (D.3) implies a smooth behavior of $R_{k F}$ for $q^{2} / k^{2} \rightarrow 0$. On the other hand, the nonanalyticity of $r_{F}$ at $q^{2}=0$ may be a source of worry for practical computations. A careful choice of $r_{F}$ is necessary in order to circumvent this problem. First we note that $r_{F}$ appears in connection with the fermion propagator from $\Gamma_{k}$ and we combine

$$
\begin{aligned}
Z_{\psi} \phi+Z_{\psi} \phi r_{F} & =Z_{\psi} \phi F \\
P_{F}=q^{2} F^{2} & =q^{2}\left(1+r_{F}\right)^{2} .
\end{aligned}
$$


Up to the wave function renormalization, $P_{F}$ corresponds to the squared inverse propagator of a free massless fermion in the presence of the infrared cutoff. We will require that $P_{F}$ and therefore $F^{2}$ is analytic in $q^{2}$ for all $q^{2} \geq 0$. A reasonable choice which we will employ in the present paper is

$$
P_{F}=P=\frac{q^{2}}{1-\exp \left\{-\frac{q^{2}}{k^{2}}\right\}} .
$$

\section{E Scalar wave function renormalization}

In this appendix we provide some details for the calculation of the flow equation of the scalar wave function renormalization $Z_{\varphi, k}(k)$. We start with the scalar contribution to (4.5). It is convenient to decompose the complex fields $\varphi_{a b}$ and $\varphi_{a b}^{\dagger}$ into their real and imaginary parts:

$$
\varphi_{a b}(q)=\frac{1}{\sqrt{2}}\left[\varphi_{R a b}(q)+i \varphi_{I a b}(q)\right], \quad \varphi_{a b}^{\dagger}(q)=\frac{1}{\sqrt{2}}\left[\varphi_{R b a}(q)-i \varphi_{I b a}(q)\right] .
$$

For the configuration (4.1) and the ansatz (3.3) it is then easy to see that $\Gamma_{S k}^{(2)}$ is blockdiagonal in the indices $R$ and $I$, i.e. it decomposes into a block $\Gamma_{R k}^{(2)}$ which contains only functional derivatives with respect to the $\varphi_{R a b}$ and an analog block $\Gamma_{I k}^{(2)}$. We may therefore use the expansion (4.8) separately for $\Gamma_{R k}^{(2)}$ and $\Gamma_{I k}^{(2)}$. The first term on the right hand side of (4.8) is independent of $\delta \varphi, \delta \varphi^{*}$ and thus can not contribute to the anomalous dimension. The part of the second term proportional to $\delta \varphi \delta \varphi^{*}$ is independent of $Q$ and hence does not contribute either to the evolution of $Z_{\varphi, k}(Q)$. Hence, we are left with the $Q$-dependent part of the third term whose contribution proportional to $\delta \varphi \delta \varphi^{*}$ is obtained by keeping all terms of $\Delta \Gamma_{R / I k}^{(2)}$ which are linear in $\Delta(q, Q)=\delta \varphi \delta(q, Q)+\delta \varphi^{*} \delta(q,-Q)$ :

$$
\begin{aligned}
{\left[\Delta \Gamma_{R k}^{(2)}\right]_{a b, c d}\left(q, q^{\prime}\right) } & =\frac{\Delta\left(q^{\prime}-q, Q\right)}{\varphi}\left\{(B+D)\left[\delta_{a d} \Sigma_{c b}+\delta_{c b} \Sigma_{a d}\right]\right. \\
& \left.+\frac{2}{N}(C-B-D)\left[\delta_{a b} \Sigma_{c d}+\delta_{c d} \Sigma_{a b}\right]\right\}+\mathcal{O}\left(\Delta^{2}\right) \\
{\left[\Delta \Gamma_{I k}^{(2)}\right]_{a b, c d}\left(q, q^{\prime}\right) } & =-\frac{\Delta\left(q^{\prime}-q, Q\right)}{\varphi}\left\{(B+D)\left[\delta_{a d} \Sigma_{c b}+\delta_{c b} \Sigma_{a d}\right]\right. \\
& \left.-D\left[\delta_{a b} \Sigma_{c d}+\delta_{c d} \Sigma_{a b}\right]\right\}+\mathcal{O}\left(\Delta^{2}\right)
\end{aligned}
$$

with

$$
B \equiv \frac{1}{2} M_{\tau}^{2}, \quad C \equiv \rho U_{k}^{\prime \prime}, \quad D \equiv \frac{1}{N} M_{\xi}^{2}
$$

From

$$
\left[\Gamma_{R k, 0}^{(2)}\right]_{a b, c d}\left(q, q^{\prime}\right)=\left\{\left(Z_{\varphi, k}(q) q^{2}+U_{k}^{\prime}\right) \delta_{a c} \delta_{b d}+B\left(\delta_{a c} \delta_{b d}+\delta_{a d} \delta_{b c}\right)\right.
$$




$$
\begin{aligned}
& \left.+\frac{2}{N}(C-B) \delta_{a b} \delta_{c d}\right\} \delta\left(q, q^{\prime}\right) \\
{\left[\Gamma_{I k, 0}^{(2)}\right]_{a b, c d}\left(q, q^{\prime}\right) } & =\left\{\left(Z_{\varphi, k}(q) q^{2}+U_{k}^{\prime}\right) \delta_{a c} \delta_{b d}+B\left(\delta_{a c} \delta_{b d}-\delta_{a d} \delta_{b c}\right)\right\} \delta\left(q, q^{\prime}\right)
\end{aligned}
$$

we obtain

$$
\begin{aligned}
\left(\Gamma_{R k, 0}^{(2)}+R_{k}\right)_{a b, c d}^{-1}\left(q, q^{\prime}\right) & =\frac{\delta\left(q, q^{\prime}\right)}{A(q)+2 B}\left\{\frac{A(q)+B}{A(q)} \delta_{a c} \delta_{b d}-\frac{B}{A(q)} \delta_{a d} \delta_{b c}\right. \\
& \left.-\frac{2}{N} \frac{C-B}{[A(q)+2 C]} \delta_{a b} \delta_{c d}\right\} \\
\left(\Gamma_{I k, 0}^{(2)}+R_{k}\right)_{a b, c d}^{-1}\left(q, q^{\prime}\right) & =\frac{\delta\left(q, q^{\prime}\right)}{A(q)}\left\{\frac{A(q)+B}{A(q)+2 B} \delta_{a c} \delta_{b d}+\frac{B}{A(q)+2 B} \delta_{a d} \delta_{b c}\right\}
\end{aligned}
$$

where

$$
A(q) \equiv Z_{\varphi, k}(q) P(q)+U_{k}^{\prime} .
$$

Now the traces can be evaluated and one finds for the scalar contribution to the scalar wave function renormalization

$$
\left[\frac{\partial}{\partial t} Z_{\varphi, k}(Q)\right]_{S}=-\frac{k^{2}}{Q^{2}} Z_{\varphi, k}^{2}(Q)\left[f_{k}^{d}(Q)-f_{k}^{d}(0)\right]
$$

with

$$
\begin{aligned}
f_{k}^{d}(Q) & =\frac{1}{2 \rho k^{2}} \int \frac{d^{d} q}{(2 \pi)^{d}} \\
& \times \frac{\widehat{\partial}}{\partial t}\left\{\frac{\left[2 \rho U_{k}^{\prime \prime}-\frac{N-2}{N} M_{\xi}^{2}\right]^{2}}{\left[Z_{\varphi, k}(q) P(q)+U_{k}^{\prime}-\frac{1}{N} M_{\xi}^{2}\right]\left[Z_{\varphi, k}(q+Q) P(q+Q)+U_{k}^{\prime}+2 \rho U_{k}^{\prime \prime}-\frac{N-1}{N} M_{\xi}^{2}\right]}\right. \\
& +\frac{N^{2}-4}{2} \frac{\left[M_{\tau}^{2}+\frac{2}{N} M_{\xi}^{2}\right]^{2}}{\left[Z_{\varphi, k}(q) P(q)+U_{k}^{\prime}-\frac{1}{N} M_{\xi}^{2}\right]\left[Z_{\varphi, k}(q+Q) P(q+Q)+U_{k}^{\prime}+M_{\tau}^{2}+\frac{1}{N} M_{\xi}^{2}\right]} \\
& \left.+\frac{\left[M_{\tau}^{2}-\frac{N-2}{N} M_{\xi}^{2}\right]^{2}}{\left[Z_{\varphi, k}(q) P(q)+U_{k}^{\prime}+\frac{N-1}{N} M_{\xi}^{2}\right]\left[Z_{\varphi, k}(q+Q) P(q+Q)+U_{k}^{\prime}+M_{\tau}^{2}+\frac{1}{N} M_{\xi}^{2}\right]}\right\}
\end{aligned}
$$

and

$$
\begin{aligned}
M_{\tau}^{2} & =\frac{4 \rho}{N-1} \frac{\partial U_{k}}{\partial \tau_{2}} \\
M_{\xi}^{2} & =-N\left(\frac{\rho}{N}\right)^{\frac{N-2}{2}} \frac{\partial U_{k}}{\partial \xi} .
\end{aligned}
$$

Here we used the fact that $P(q)$ and $Z_{\varphi, k}(q)$ are actually functions of the invariant $q^{2}$.

We turn next to the fermionic contribution to the scalar anomalous dimension. Analogously to the scalar contribution we may split $\Gamma_{F k}^{(2)}$ into $\Gamma_{F k, 0}^{(2)}$ and $\Delta \Gamma_{F k}^{(2)}$ which contains 
all dependence on the scalar background fluctuation $\delta \varphi$. For the truncation (3.3) of the average action we obtain

$$
\begin{aligned}
\left(\Gamma_{F k, 0}^{(2)}+R_{F k}\right)_{a b}^{-1}\left(q, q^{\prime}\right) & =\frac{Z_{\psi, k}(q)\left[1+r_{F}(q)\right] \phi+\bar{h}_{k}(q) \varphi \bar{\gamma}}{Z_{\psi, k}^{2}(q) P_{F}(q)+\bar{h}_{k}^{2}(q) \varphi^{2}} \delta_{a b} \delta\left(q, q^{\prime}\right) \\
\left(\Delta \Gamma_{F k}^{(2)}\right)_{a b}\left(q, q^{\prime}\right) & =\bar{h}_{k}\left(-q, q^{\prime}\right) \Delta\left(q^{\prime}-q, Q\right) \Sigma_{a b}
\end{aligned}
$$

where we used the abbreviation $\bar{h}_{k}(q) \equiv \bar{h}_{k}(-q, q)$. Using an expansion similar to (4.8) the fermionic trace can be evaluated and we obtain its contribution to the scalar anomalous dimension by collecting all $Q$-dependent terms of order $\delta \varphi \delta \varphi^{*}$. One finds

$$
\left[\frac{\partial}{\partial t} Z_{\varphi, k}(Q)\right]_{F}=-2^{\frac{d}{2}-1} N_{c} \frac{k^{2}}{Q^{2}}\left[f_{F k}^{d}(Q)-f_{F k}^{d}(0)\right]
$$

with

$$
\begin{aligned}
f_{F k}^{d}(Q) & =k^{-2} \int \frac{d^{d} q}{(2 \pi)^{d}}\left(q^{2}+q Q\right) \bar{h}_{k}^{2}(-q, q+Q) \\
& \left.\times \widehat{\partial} \frac{Z_{\psi, k}(q)\left[1+r_{F}(q)\right]}{\left[Z_{\psi, k}^{2}(q) P_{F}(q)+\frac{1}{N} \bar{h}_{k}^{2}(q) \rho\right]} \frac{Z_{\psi, k}(q+Q)\left[1+r_{F}(q+Q)\right]}{\left[Z_{\psi, k}^{2}(q+Q) P_{F}(q+Q)+\frac{1}{N} \bar{h}_{k}^{2}(q+Q) \rho\right]}\right\} .
\end{aligned}
$$

and we made use of the identity $\bar{h}_{k}\left(-q, q^{\prime}\right)=\bar{h}_{k}\left(-q^{\prime}, q\right)$.

The fermionic contribution to $\eta_{\varphi}$ obtains from the term linear in $Q^{2}$ in $f_{F k}^{d}$ in close analogy to [23], and similarly for the scalar contribution, where we recover the results of [10] for $\lambda_{2}=\nu=0$. Taking $\rho$ at the minimum of the potential and neglecting all momentum dependence of the Yukawa coupling and wave function renormalizations we arrive at

$$
\begin{aligned}
\eta_{\varphi} & =4 \frac{v_{d}}{d} \kappa\left\{\left[2 \lambda_{1}-\frac{N-2}{2} \frac{\nu}{N}\left(\frac{\kappa}{N}\right)^{\frac{N-4}{2}}\right]^{2} m_{2,2}^{d}\left(0,2 \kappa \lambda_{1}-\frac{N-2}{2} \nu\left(\frac{\kappa}{N}\right)^{\frac{N-2}{2}} ; \eta_{\varphi}\right)\right. \\
& +\left[\lambda_{2}-\frac{N-2}{2} \frac{\nu}{N}\left(\frac{\kappa}{N}\right)^{\frac{N-4}{2}}\right]^{2} m_{2,2}^{d}\left(\kappa \lambda_{2}+\nu\left(\frac{\kappa}{N}\right)^{\frac{N-2}{2}}, \frac{N}{2} \nu\left(\frac{\kappa}{N}\right)^{\frac{N-2}{2}} ; \eta_{\varphi}\right) \\
& \left.+\frac{N^{2}-4}{2}\left[\lambda_{2}+\frac{\nu}{N}\left(\frac{\kappa}{N}\right)^{\frac{N-4}{2}}\right]^{2} m_{2,2}^{d}\left(0, \kappa \lambda_{2}+\nu\left(\frac{\kappa}{N}\right)^{\frac{N-2}{2}} ; \eta_{\varphi}\right)\right\} \\
& +2^{\frac{d}{2}+2} \frac{v_{d}}{d} N_{c} h^{2} m_{4}^{(F) d}\left(\frac{1}{N} \kappa h^{2} ; \eta_{\psi}\right) .
\end{aligned}
$$

The threshold functions $m_{n_{1}, n_{2}}^{d}$ and $m_{4}^{(F) d}$ are defined in (4.11) and (4.12), respectively. 


\section{F Yukawa coupling and fermion wave function renor- malization}

We will give here details on the derivation of the evolution equations for the fermionic wave function renormalization constant and the Yukawa coupling as defined in section 5. We use here the truncation (3.3) but neglect for simplicity the dependence of the Yukawa coupling on the scalar momentum and also the momentum dependence of $Z_{\varphi}$. For the scalar fields it proves useful to introduce the following linear combinations:

$$
\begin{aligned}
\varphi_{R a} & =\frac{1}{\sqrt{2}}\left(\varphi_{a a}+\varphi_{a a}^{*}\right) \\
\varphi_{I a} & =\frac{-i}{\sqrt{2}}\left(\varphi_{a a}-\varphi_{a a}^{*}\right)
\end{aligned}
$$

and for $a \neq b$

$$
\begin{aligned}
\varphi_{R+a b} & =\frac{1}{2}\left(\varphi_{a b}+\varphi_{b a}+\varphi_{a b}^{*}+\varphi_{b a}^{*}\right) \\
\varphi_{R-a b} & =\frac{1}{2}\left(\varphi_{a b}-\varphi_{b a}+\varphi_{a b}^{*}-\varphi_{b a}^{*}\right) \\
\varphi_{I+a b} & =\frac{-i}{2}\left(\varphi_{a b}+\varphi_{b a}-\varphi_{a b}^{*}-\varphi_{b a}^{*}\right) \\
\varphi_{I-a b} & =\frac{-i}{2}\left(\varphi_{a b}-\varphi_{b a}-\varphi_{a b}^{*}+\varphi_{b a}^{*}\right)
\end{aligned}
$$

Using collective indices $i, j \in\{R a, I a, R+a b, R-a b, I+a b, I-a b\}$ this yields

$$
\frac{\delta^{2} \Gamma_{k}}{\delta \varphi_{i}^{*}(q) \delta \varphi^{j}\left(q^{\prime}\right)}=\left(Z_{\varphi} q^{2} \delta_{j}^{i}+\left(M^{2}\right)_{j}^{i}\right)(2 \pi)^{d} \delta\left(q-q^{\prime}\right)
$$

with scalar mass matrix

$$
\left(M^{2}\right)_{j}^{i}=\frac{\partial^{2} U_{k}}{\partial \varphi_{i}^{*} \partial \varphi^{j}} .
$$

This matrix is discussed in appendix B and does not mix the various fields (F.1), (F.2). The mixed functional derivatives of $\Gamma_{k}$ with respect to one scalar and one fermion field are easily seen to be of first order in the fermion fields. Since we are interested in the term bilinear in $\psi$, we can take $\psi$ to be infinitesimally small and split

$$
\Gamma_{k}^{(2)}=\Gamma_{k, 0}^{(2)}+\Delta \Gamma_{k}^{(2)}
$$

in such a way that all $\psi$-dependence is entailed in $\Delta \Gamma_{k}^{(2)}$. Hence $\Gamma_{k, 0}^{(2)}$ is given by (5.3), (F.3) and $\Delta \Gamma_{k}^{(2)}$ is determined by the mixed scalar-fermionic functional derivatives of $\Gamma_{k}$. Using an expansion similar to (4.8) we arrive at the following evolution equation for the 
bilinear fermionic part $\Gamma_{k, 2}^{(\psi)}$ of the effective average action:

$$
\begin{aligned}
\frac{\partial}{\partial t} \Gamma_{k, 2}^{(\psi)} & =\frac{1}{2 N} \int \frac{d^{d} Q}{(2 \pi)^{d}} \int \frac{d^{d} q}{(2 \pi)^{d}} \bar{h}_{k}^{2}\left(\frac{q+Q}{2}\right) \frac{\widehat{\partial}}{\partial t}\left\{\frac{Z_{\psi, k}(q) \bar{\psi}_{a}(Q) \phi\left[1+r_{F}(q)\right] \psi^{a}(Q)}{Z_{\psi, k}^{2}(q) P_{F}(q)+\bar{h}_{k}^{2}(q) \varphi^{2}}\right. \\
& \times\left(\frac{N^{2}-1}{Z_{\varphi} P(q-Q)+U_{k}^{\prime}-\frac{1}{N} M_{\xi}^{2}}+\frac{N^{2}-1}{Z_{\varphi} P(q-Q)+U_{k}^{\prime}+M_{\tau}^{2}+\frac{1}{N} M_{\xi}^{2}}\right. \\
& \left.+\frac{1}{Z_{\varphi} P(q-Q)+U_{k}^{\prime}+2 \rho U_{k}^{\prime \prime}-\frac{N-1}{N} M_{\xi}^{2}}+\frac{1}{Z_{\varphi} P(q-Q)+U_{k}^{\prime}+\frac{N-1}{N} M_{\xi}^{2}}\right) \\
& +\frac{\bar{h}_{k}(q) \varphi \bar{\psi}^{a}(Q) \bar{\gamma} \psi_{a}(Q)}{Z_{\psi, k}^{2}(q) P_{F}(q)+\bar{h}_{k}^{2}(q) \varphi^{2}} \\
& \times\left(\frac{N^{2}-1}{Z_{\varphi} P(q-Q)+U_{k}^{\prime}-\frac{1}{N} M_{\xi}^{2}}-\frac{1}{Z_{\varphi} P(q-Q)+U_{k}^{\prime}+M_{\tau}^{2}+\frac{1}{N} M_{\xi}^{2}}\right. \\
& \left.\left.-\frac{1}{Z_{\varphi} P(q-Q)+U_{k}^{\prime}+2 \rho U_{k}^{\prime \prime}-\frac{N-1}{N} M_{\xi}^{2}}+\frac{1}{Z_{\varphi} P(q-Q)+U_{k}^{\prime}+\frac{N-1}{N} M_{\xi}^{2}}\right)\right\}
\end{aligned}
$$

with $M_{\tau}^{2}=\frac{4 \rho}{N-1} \frac{\partial U_{k}}{\partial \tau_{2}}$ and $M_{\xi}^{2}=-N\left(\frac{\rho}{N}\right)^{\frac{N-2}{2}} \frac{\partial U_{k}}{\partial t}$. It is easy to extract from (F.6) the evolution equations for the Yukawa coupling and the fermionic anomalous dimension. For the Yukawa coupling, renormalized according to (5.2), one finds for $\rho$ at the minimum of the potential

$$
\begin{aligned}
\frac{\partial}{\partial t} h_{k}^{2}(Q) & =\left[d-4+2 \eta_{\psi, k}(Q)+\eta_{\varphi, k}\right] h_{k}^{2}(Q) \\
& +\frac{k^{4-d}}{N} h_{k}(Q) \int \frac{d^{d} q}{(2 \pi)^{d}} h_{k}(q) h_{k}^{2}\left(\frac{q+Q}{2}\right) \frac{Z_{\psi, k}^{2}\left(\frac{q+Q}{2}\right)}{Z_{\psi, k}(q) Z_{\psi, k}(Q)} Z_{\varphi} \\
& \times \frac{\widehat{\partial}}{\partial t}\left\{\frac { 1 } { P _ { F } ( q ) + \frac { k ^ { 2 } } { N } h _ { k } ^ { 2 } ( q ) \kappa } \left[\frac{N^{2}-1}{Z_{\varphi} P(q-Q)+U_{k}^{\prime}-\frac{1}{N} M_{\xi}^{2}}\right.\right. \\
& +\frac{1}{Z_{\varphi} P(q-Q)+U_{k}^{\prime}+\frac{N-1}{N} M_{\xi}^{2}}-\frac{N^{2}-1}{Z_{\varphi} P(q-Q)+U_{k}^{\prime}+M_{\tau}^{2}+\frac{1}{N} M_{\xi}^{2}} \\
& \left.\left.-\frac{1}{Z_{\varphi} P(q-Q)+U_{k}^{\prime}+2 \rho U_{k}^{\prime \prime}-\frac{N-1}{N} M_{\xi}^{2}}\right]\right\} .
\end{aligned}
$$


Analogously we obtain for the fermion anomalous dimension

$$
\begin{aligned}
\eta_{\psi, k}(Q) & =-\frac{k^{4-d}}{2 N} \int \frac{d^{d} q}{(2 \pi)^{d}} \frac{q Q}{Q^{2}} h_{k}^{2}\left(\frac{q+Q}{2}\right) \frac{Z_{\psi, k}^{2}\left(\frac{q+Q}{2}\right) Z_{\varphi}}{Z_{\psi, k}(q) Z_{\psi, k}(Q)} \frac{\widehat{\partial}}{\partial t}\left\{\frac{\left[1+r_{F}(q)\right]}{P_{F}(q)+\frac{k^{2}}{N} h_{k}^{2}(q) \kappa}\right. \\
& \times\left(\frac{N^{2}-1}{Z_{\varphi} P(q-Q)+U_{k}^{\prime}-\frac{1}{N} M_{\xi}^{2}}+\frac{1}{Z_{\varphi} P(q-Q)+U_{k}^{\prime}+\frac{N-1}{N} M_{\xi}^{2}}\right. \\
& +\frac{N^{2}-1}{Z_{\varphi} P(q-Q)+U_{k}^{\prime}+M_{\tau}^{2}+\frac{1}{N} M_{\xi}^{2}} \\
& \left.\left.+\frac{1}{Z_{\varphi} P(q-Q)+U_{k}^{\prime}+2 \rho U_{k}^{\prime \prime}-\frac{N-1}{N} M_{\xi}^{2}}\right)\right\} .
\end{aligned}
$$

In the following we do not discuss further the momentum dependence in the fermionic sector. We are interested in the evolution of $\eta_{\psi}=\eta_{\psi, k}(0)$ and $h^{2}=h_{k}^{2}(0)$. Neglecting the momentum dependence of $Z_{\psi, k}$ and $h_{k}$ on the right hand side of (F.7) and (F.8) and taking the limit $Q^{2} \rightarrow 0$ we obtain

$$
\begin{aligned}
\frac{\partial}{\partial t} h^{2}= & {\left[d-4+2 \eta_{\psi}+\eta_{\varphi}\right] h^{2}-\frac{4}{N} v_{d} h^{4}\left\{\left(N^{2}-1\right)^{2} l_{1,1}^{(F B) d}\left(\frac{1}{N} \kappa h^{2}, \epsilon ; \eta_{\psi}, \eta_{\varphi}\right)\right] } \\
+ & l_{1,1}^{(F B) d}\left(\frac{1}{N} \kappa h^{2}, \epsilon+\frac{N}{2} \nu\left(\frac{\kappa}{N}\right)^{\frac{N-2}{2}} ; \eta_{\psi}, \eta_{\varphi}\right) \\
- & \left(N^{2}-1\right) l_{1,1}^{(F B) d}\left(\frac{1}{N} \kappa h^{2}, \epsilon+\kappa \lambda_{2}+\nu\left(\frac{\kappa}{N}\right)^{\frac{N-2}{2}} ; \eta_{\psi}, \eta_{\varphi}\right) \\
- & \left.l_{1,1}^{(F B) d}\left(\frac{1}{N} \kappa h^{2}, \epsilon+2 \kappa \lambda_{1}-\frac{N-2}{2} \nu\left(\frac{\kappa}{N}\right)^{\frac{N-2}{2}} ; \eta_{\psi}, \eta_{\varphi}\right)\right\} \\
\eta_{\psi}= & \frac{4}{N} \frac{v_{d}}{d} h^{2}\left\{\left(N^{2}-1\right) m_{1,2}^{(F B) d}\left(\frac{1}{N} h^{2} \kappa, \epsilon ; \eta_{\psi}, \eta_{\varphi}\right)\right. \\
& +m_{1,2}^{(F B) d}\left(\frac{1}{N} h^{2} \kappa, \epsilon+\frac{N}{2} \nu\left(\frac{\kappa}{N}\right)^{\frac{N-2}{2}} ; \eta_{\psi}, \eta_{\varphi}\right) \\
& +\left(N^{2}-1\right) m_{1,2}^{(F B) d}\left(\frac{1}{N} h^{2} \kappa, \epsilon+\kappa \lambda_{2}+\nu\left(\frac{\kappa}{N}\right)^{\frac{N-2}{2}} ; \eta_{\psi}, \eta_{\varphi}\right) \\
& \left.+m_{1,2}^{(F B) d}\left(\frac{1}{N} h^{2} \kappa, \epsilon+2 \kappa \lambda_{1}-\frac{N-2}{2} \nu\left(\frac{\kappa}{N}\right)^{\frac{N-2}{2}} ; \eta_{\psi}, \eta_{\varphi}\right)\right\} .
\end{aligned}
$$

The threshold functions $l_{n_{1}, n_{2}}^{(F B) d}$ and $m_{n_{1}, n_{2}}^{(F B) d}$ are defined in (5.5) and (5.7), respectively. In the symmetric regime one has to set $\kappa=0$ in both expressions, whereas in the SSB regime $\epsilon=0$. 


\section{G Threshold integrals}

In this appendix we provide explicit expressions for the threshold integrals introduced in the previous sections which are suitable for a direct numerical integration. For this purpose we introduce the dimensionless integration variable $y=q^{2} / k^{2}=x / k^{2}$. Using the abbreviations

$$
\begin{array}{ll}
p \equiv p(y)=k^{-2} P(x) \quad, \quad \dot{p} \equiv \dot{p}(y)=\frac{\partial p(y)}{\partial y} \quad, \quad \text { etc. } \\
p_{F} \equiv p_{F}(y)=k^{-2} P_{F}(x) \quad, \quad \dot{p}_{F} \equiv \dot{p}_{F}(y)=\frac{\partial p_{F}(y)}{\partial y} \quad, \quad \text { etc. } \\
r_{F} \equiv r_{F}(y)=\sqrt{\frac{p_{F}(y)}{y}}-1 \quad, \quad \dot{r}_{F} \equiv \dot{r}_{F}(y)=\frac{\partial r_{F}(y)}{\partial y} \quad, \quad \text { etc. }
\end{array}
$$

we find

$$
\begin{aligned}
& l_{n}^{d}(w)=n \int_{0}^{\infty} d y y^{\frac{d}{2}-1} \frac{p-y \dot{p}}{[p+w]^{n+1}} \\
& \hat{l}_{n}^{d}(w)=\frac{n}{2} \int_{0}^{\infty} d y y^{\frac{d}{2}-1} \frac{p-y}{[p+w]^{n+1}} \\
& l_{n}^{(F) d}(w)=n \int_{0}^{\infty} d y y^{\frac{d}{2}-1} \frac{p_{F}-y \dot{p}_{F}}{\left[p_{F}+w\right]^{n+1}} \\
& \check{l}_{n}^{(F) d}(w)=n \int_{0}^{\infty} d y y^{\frac{d}{2}} \frac{r_{F}\left[r_{F}+1\right]}{\left[p_{F}+w\right]^{n+1}} \\
& l_{n_{1}, n_{2}}^{d}\left(w_{1}, w_{2}\right)=\int_{0}^{\infty} d y y^{\frac{d}{2}-1}(p-y \dot{p}) \\
& \times\left\{\frac{n_{1}}{\left[p+w_{1}\right]^{n_{1}+1}\left[p+w_{2}\right]^{n_{2}}}+\frac{n_{2}}{\left[p+w_{1}\right]^{n_{1}}\left[p+w_{2}\right]^{n_{2}+1}}\right\} \\
& \hat{l}_{n_{1}, n_{2}}^{d}\left(w_{1}, w_{2}\right)=\frac{1}{2} \int_{0}^{\infty} d y y^{\frac{d}{2}-1}(p-y) \\
& \times\left\{\frac{n_{1}}{\left[p+w_{1}\right]^{n_{1}+1}\left[p+w_{2}\right]^{n_{2}}}+\frac{n_{2}}{\left[p+w_{1}\right]^{n_{1}}\left[p+w_{2}\right]^{n_{2}+1}}\right\} \\
& l_{n_{1}, n_{2}}^{(F B) d}\left(w_{1}, w_{2}\right)=\int_{0}^{\infty} d y y^{\frac{d}{2}-1} \frac{1}{\left[p_{F}+w_{1}\right]^{n_{1}}\left[p+w_{2}\right]^{n_{2}}} \\
& \times\left\{\frac{n_{1}\left[p_{F}-y \dot{p}_{F}\right]}{\left[p_{F}+w_{1}\right]}+\frac{n_{2}[p-y \dot{p}]}{\left[p+w_{2}\right]}\right\} \\
& \hat{l}_{n_{1}, n_{2}}^{(F B) d}\left(w_{1}, w_{2}\right)=\frac{n_{2}}{2} \int_{0}^{\infty} d y y^{\frac{d}{2}-1} \frac{p-y}{\left[p_{F}+w_{1}\right]^{n_{1}}\left[p+w_{2}\right]^{n_{2}+1}} \\
& \check{l}_{n_{1}, n_{2}}^{(F B) d}\left(w_{1}, w_{2}\right)=n_{1} \int_{0}^{\infty} d y y^{\frac{d}{2}} \frac{r_{F}\left[r_{F}+1\right]}{\left[p_{F}+w_{1}\right]^{n_{1}+1}\left[p+w_{2}\right]^{n_{2}}}
\end{aligned}
$$




$$
\begin{aligned}
& m_{n_{1} n_{2}}^{d}\left(w_{1}, w_{2}\right)=\int_{0}^{\infty} d y y^{\frac{d}{2}} \frac{\dot{p}}{\left[p+w_{1}\right]^{n_{1}}\left[p+w_{2}\right]^{n_{2}}} \\
& \times\left\{\frac{n_{1} \dot{p}[p-y \dot{p}]}{\left[p+w_{1}\right]}+\frac{n_{2} \dot{p}[p-y \dot{p}]}{\left[p+w_{2}\right]}+2 y \ddot{p}\right\} \\
& \hat{m}_{n_{1} n_{2}}^{d}\left(w_{1}, w_{2}\right)=\frac{1}{2} \int_{0}^{\infty} d y y^{\frac{d}{2}} \frac{\dot{p}}{\left[p+w_{1}\right]^{n_{1}}\left[p+w_{2}\right]^{n_{2}}} \\
& \times\left\{\frac{n_{1} \dot{p}[p-y]}{\left[p+w_{1}\right]}+\frac{n_{2} \dot{p}[p-y]}{\left[p+w_{2}\right]}+2[1-\dot{p}]\right\} \\
& m_{4}^{(F) d}(w)=2 \int_{0}^{\infty} d y y^{\frac{d}{2}+1} \frac{1}{\left[p_{F}+w\right]^{3}}\left[\frac{\dot{p}\left[1+r_{F}\right]}{p_{F}+w}-\dot{r}_{F}\right] \\
& \times\left\{\left[\dot{r}_{F}+y \ddot{r}_{F}\right]\left[p_{F}-w\right]-y \dot{r}_{F} \dot{p}_{F} \frac{p_{F}-3 w}{p_{F}+w}\right\} \\
& \check{m}_{4}^{(F) d}(w)=\int_{0}^{\infty} d y y^{\frac{d}{2}+1} \frac{1}{\left[p_{F}+w\right]^{3}}\left[\frac{\dot{p}\left[1+r_{F}\right]}{p_{F}+w}-\dot{r}_{F}\right] \\
& \times\left\{\dot{r}_{F}\left[p_{F}-w\right]-r_{F} \dot{p}_{F} \frac{p_{F}-3 w}{p_{F}+w}\right\} \\
& m_{n_{1}, n_{2}}^{(F B)}\left(w_{1}, w_{2}\right)=\int_{0}^{\infty} d y y^{\frac{d}{2}} \frac{1}{\left[p_{F}+w_{1}\right]^{n_{1}}\left[p+w_{2}\right]^{n_{2}}} \\
& \times\left\{\left[1+r_{F}\right]\left(n_{2} \frac{\dot{p}[p-y \dot{p}]}{p+w_{2}}+y \ddot{p}\right)-y \dot{p}_{F}\left(\frac{2 n_{1} p_{F}}{p_{F}+w_{1}}-1\right)\right\} \\
& \hat{m}_{n_{1}, n_{2}}^{(F B) d}\left(w_{1}, w_{2}\right)=\frac{1}{2} \int_{0}^{\infty} d y y^{\frac{d}{2}} \frac{\left[1+r_{F}\right]}{\left[p_{F}+w_{1}\right]^{n_{1}}\left[p+w_{2}\right]^{n_{2}}} \\
& \times\left\{n_{2} \frac{\dot{p}[p-y]}{p+w_{2}}+1-\dot{p}\right\} \\
& \check{m}_{n_{1}, n_{2}}^{(F B) d}\left(w_{1}, w_{2}\right)=\frac{n_{1}}{2} \int_{0}^{\infty} d y y^{\frac{d}{2}} \frac{r_{F} \dot{p}}{\left[p_{F}+w_{1}\right]^{n_{1}+1}\left[p+w_{2}\right]^{n_{2}}}\left(\frac{2 n_{1} p_{F}}{p_{F}+w_{1}}-1\right)
\end{aligned}
$$




\section{References}

[1] D. J. Gross and F. A. Wilczek, Phys. Rev. Lett. 30 (1973) 1343;

H. D. Politzer, Phys. Rev. Lett. 30 (1973) 1346.

[2] S. Weinberg, Physica A96 (1979) 327.

[3] J. Gasser and H. Leutwyler, Phys. Rep. C87 (1982) 77; Nucl. Phys. B250 (1985) 465.

[4] M. Gell-Mann and M. Levy, Nuovo Cim. 16 (1960) 705

[5] L. Reinders, H. Rubinstein and S. Yazaki, Phys. Rep. C127 (1985)

[6] M. Lüscher, R. Sommer, P. Weisz and U. Wolf, Nucl. Phys. B413 (1994) 481

[7] Y. Nambu and G. Jona-Lasinio, Phys. Rev. 122 (1961) 345;

D. Gromes, Z. Phys. C11 (1981) 147;

R. Haymaker, Riv. Nuovo Cim. 14 (1991) No. 8;

J. Bijnens, preprint NORDITA 95/10 N,P (hep-ph/9502335).

[8] C. Wetterich, Nucl. Phys. B352 (1991) 529; Z. Phys. C60 (1993) 461.

[9] C. Wetterich, Phys. Lett. 301B (1993) 90.

[10] C. Wetterich, Z. Phys. C57 (1993) 451.

[11] M. Bonini, M. D'Attanasio, and G. Marchesini, Nucl. Phys. B409 (1993) 441;

U. Ellwanger, Z. Phys. C58 (1993) 619;

C. Wetterich, Int. J. Mod. Phys. A9 (1994) 3571.

[12] F. Wegner and A. Houghton, Phys. Rev. A8 (1973) 401;

K. G. Wilson and I. G. Kogut, Phys. Rev. 12 (1974) 74;

S. Weinberg, Critical Phenomena for Field Theorists, Erice Subnucl. Phys. (1976);

J. Polchinski, Nucl. Phys. B231 (1984) 269;

A. Hasenfratz and P. Hasenfratz, Nucl. Phys. B270 (1986) 685.

[13] F. J. Dyson, Phys. Rev. 75 (1949) 1736;

J. Schwinger, Proc. Nat. Acad. Sc. 37 (1951) 452, 455.

[14] U. Ellwanger and C. Wetterich, Nucl. Phys. B423 (1994) 137.

[15] C. Wetterich, Heidelberg preprint HD-THEP-95-2

[16] R.S. Chivukula, M. Golden and E.H. Simmons, Phys. Rev. Lett. 70 (1993) 1587

[17] W.A. Bardeen, C.T. Hill and D.-U. Jungnickel, Phys. Rev. D49 (1994) 1437 
[18] Y. Nambu, EFI preprints 89-08 and 89-39;

V. Miransky, M. Tanabashi, and K. Yamawaki, Phys. Lett. 221B (1989) 177; Mod. Phys. Lett. A4 (1989) 1043;

W. A. Bardeen, C. Hill, and M. Lindner, Phys. Rev. D41 (1991) 1647;

A. Hasenfratz, P. Hasenfratz, K. Jansen, J. Kuti and Y. Shen, Nucl. Phys. B365 (1991) 79 ;

U. Ellwanger and L. Vergara, Nucl. Phys. B398 (1993) 52.

[19] N. Tetradis and C. Wetterich, Nucl. Phys. B398 (1993) 659; Int. J. Mod. Phys. A9 (1994) 4029.

[20] R.D. Pisarski and F. Wilczek, Phys. Rev. D29 (1984) 338;

K. Rajagopal and F. Wilczek, Nucl. Phys. B399 (1993) 395; Nucl. Phys. B399 (1993) 577.

[21] K. Geiger, CERN preprint CERN-TH-7440-94 (hep-ph/9409309);

K. Geiger and J. Ellis, CERN preprint CERN-TH-95-34 (hep-ph/9503212).

[22] C. Wetterich, Z. Phys. C48 (1990) 693.

[23] S. Bornholdt and C. Wetterich, Z. Phys. C58 (1993) 585.

[24] S. Bornholdt, N. Tetradis and C. Wetterich, Phys. Lett. 348B (1995) 89.

[25] C. Wetterich, Phys. Lett. 104B (1981) 269.

[26] C.T. Hill, Phys. Rev. D24 (1981) 691;

C.Wetterich in Superstrings, Unified Theories and Cosmology, eds G. Furlan, J.C. Pati, D.W. Sciama, E. Szegin and Q. Shafi, World Scientific (1988) 403.

[27] M. Jamin and M. Münz, preprint CERN-TH.7435/94

[28] M. Reuter and C. Wetterich, Nucl. Phys. B391 (1993) 147; B408 (1993) 91; B417 (1994) 181; B427 (1994) 291; Heidelberg preprint HD-THEP-94-39;

M. Bonini, M. D'Attanasio, and G. Marchesini, Nucl. Phys. B418 (1994) 81; B421 (1994) 429;

U. Ellwanger, Phys. Lett. 335B (1994) 364. 